\title{
4 Zur Handlungssituation des Krankenhausmanagements
}

\subsection{Einführung und Überblick}

Die Entwicklung eines Management-Konzepts, das sozial verantwortliches Handeln von Führungskräften und Mitarbeitern verlangt und das die Voraussetzungen dafür schafft, dass Führungskräfte und Mitarbeiter dieses Konzept akzeptieren und praktizieren wollen und können, erfolgt auf der Crundlage der Handlungssituation des Krankenhausmanagements. In dieser kommen die kulturellen und strukturellen Besonderheiten und andere Bedingungen der Leitungsarbeit eines Krankenhauses zum Ausdruck. Sie markiert den Handlungsspielraum, der es den Verantwortungsträgern entweder ermöglicht, bei ihren Entscheidungen die Anliegen der von diesen Entscheidungen betroffenen angemessen $\mathrm{zu}$ berücksichtigen, oder der dieses verunmöglicht, wenn die Grenzen des Handlungsspielraums allzu eng gezogen werden.

Die aktuelle Handlungssituation eines Krankenhauses ist als Basis für sozial verantwortliches Handeln nicht immer oder nur bedingt geeignet und muss deshalb möglicherweise geändert werden (Beispiel 1).
Beispiel 1

Eine zentralistisch geprägte Leitungsstruktur konzentriert einen großen Teil der Leitungsbefugnisse bei Stellen und/ oder Gremien, die in der Spitze des Unternehmens verortet sind. Führungskräfte und Mitarbeiter in den nachgeordneten Leitungsebenen sind überwiegend ausführend tätig.

Der Umfang der den zuletzt genannten Führungskräften und Mitarbeitern zugewiesenen Entscheidungsspielräume tendiert gegen Null. Sie haben deshalb keine Möglichkeit, Stakeholder-Anliegen, dazu zählen unter anderem auch die Bedürfnisse der ihnen nachgeordneten Führungskräfte und Mitarbeiter, bei den von innen zu fällenden Entscheidungen angemessen zu berücksichtigen.

Eine derartig organisierte Leitung eines Krankenhauses ist für das Wirksamwerden eines Konzeptes des Managements der sozialen Verantwortung nur bedingt, wenn überhaupt geeignet. Die Leitungsstruktur wird angepasst werden müssen, damit die Akteure des Krankenhauses sozial verantwortlich handeln können.

Umso wichtiger ist es, die gegebene Handlungssituation zu analysieren und vor allem auch die Motive für deren Gestaltung zu identifizieren und zu bewerten. Erst danach ist man imstande festzulegen, welche Elemente der aktuellen Handlungssituation in das neue Management-Konzept 
übernommen werden können und welche gegebenenfalls neu gefasst werden müssen.

Das für das konkrete Unternehmen geeignete Konzept eines Managements der sozialen Verantwortung kann nur unter Berücksichtigung der für dieses Unternehmen spezifischen Handlungssituation und unter aktiver Beteiligung der Geschäftsführung entwickelt und implementiert werden. Wenn die Geschäftsführung eines Krankenhauses allerdings meint, sich dieser Mühe nicht unterziehen zu müssen und ein Konzept des Managements der sozialen Verantwortung „von der Stange“ kauft, besteht die Gefahr, dass Elemente, also zum Beispiel Normen für den Umgang der Verantwortungsträger mit den Adressaten der Verantwortung, in das neue Konzept eingeführt werden, die die spezifischen, nicht selten unsichtbaren Bedingungen der Leitungsarbeit in dem konkreten Krankenhaus nicht abbilden und deshalb auch nicht realisiert werden können. Möglicherweise werden organisationale Barrieren, die die Wahrnehmung von Verantwortung verunmöglichen, nicht erkannt und deshalb auch nicht abgebaut.
In den folgenden Kapiteln wird die Handlungssituation eines Krankenhauses beispielhaft beschrieben. Diese Analyse ist als Anregung - als Checkliste - für die erforderliche Analyse im konkreten Entwicklungs- und Implementierungsfall gedacht. Sie darf Letztere nicht ersetzen. Für das konkrete Krankenhaus würde sonst - wie schon erwähnt - möglicherweise ein Management der sozialen Verantwortung konzipiert und praktiziert werden, das für diesen Einzelfall nicht geeignet ist und deshalb zu Fehlentwicklungen beitragen kann.

Die Handlungssituation des Krankenhausmanagements wird durch Faktoren geprägt, die, wenn sie sich verändern, im Regelfall Anlass für unternehmerisches Handeln sind. Dabei geht es um (s. Abb. 21)

- externe Einflussfaktoren

- diverse die Leitungsarbeit treibende Faktoren,

- diverse, eher restriktiv wirkende Rahmenbedingungen,

- interne Einflussfaktoren

- das Leistungsprogramm,



Abb. 21 Diverse Einflussfaktoren sowie Stakeholder-Anliegen prägen unternehmerische Entscheidungen 
- diverse konstitutive Bedingungen der Leitungsarbeit - dazu gehört auch die Unternehmensorganisation (= Leitungsstruktur des Krankenhauses und die Gestaltung der Entscheidungsprozesse) (Näheres dazu in Kap. 6),

n für die Leitungsarbeit in Krankenhäusern typische Produktions- und kulturelle Bedingungen sowie um

- Anliegen und Beiträge diverser Stakeholder.

Wenn die Ergebnisse einer HandlungssituationsAnalyse helfen sollen, sozial verantwortliches Handeln dauerhaft wirksam werden zu lassen, dann werden Antworten auf die nachstehend genannten Fragen benötigt:

1. Welche Einflussfaktoren und sonstige Faktoren mit welcher Charakteristik sind für die Entwicklung des Konzepts des Managements der sozialen Verantwortung prägend?

2. Welche Beziehungen gibt es zwischen den Elementen der in der Abbildung 21 skizzierten Handlungssituation?

3. Welchen Zusammenhang gibt es zwischen möglichen Einflussfaktoren-Veränderungen und der Dimension des dem Verantwortungsträger zur Verfügung stehenden Handlungsspielraums?

4. Welche Rollen spielen die Stakeholder?

5. Welche Beziehungen gibt es zwischen den Zielen, die Stakeholder mittels ihrer Anliegen verfolgen?
Der Begründung und Erläuterung dieser Fragen dienen die nun folgenden Ausführungen. Die beispielhafte Beantwortung der Fragen im Detail erfolgt in den Kapiteln 4.2, 4.3 und 4.4.

\section{Zu 1. Welche Einflussfaktoren und sonstige Faktoren mit welcher Charakteristik sind für die Entwicklung des Konzepts des Managements der sozialen Verantwortung prägend?}

Im Sinne der Grundidee des Managements der sozialen Verantwortung ist die Identifizierung und Charakterisierung der Stakeholder sowie die Analyse und Bewertung der Stakeholder-Anliegen und -Beiträge von zentraler Bedeutung. Es empfiehlt sich deshalb, den Analyseprozess mit dem Erstellen einer möglichst vollständigen Liste der für das Krankenhaus relevanten Stakeholder (s. Tab. 4) zu beginnen. In dieser Liste sollten die Anliegen und Beiträge der Stakeholder möglichst detailliert beschrieben werden, um sie bewerten und um anschließend entscheiden zu können, ob und mit welchem Gewicht sie bei Entscheidungen berücksichtigt werden sollen.

Die Vorarbeiten werden fortgesetzt mit der Auflistung und Beschreibung aller jener Faktoren, die die Leitungsarbeit in einem Krankenhaus entweder treiben oder restriktiv beeinflussen. Dem zur Verfügung stehenden Platz geschuldet, werden von diesen Faktoren in den Kapiteln 4.2 bis 4.4 nur die Produktions- und kulturellen Bedingungen der Leitungsarbeit (s. Kap. 4.2), die externen Einflussfaktoren (die Leitungsarbeit treibenden Faktoren [s. Kap. 4.3] und Rahmenbedingungen [s. Kap. 4.4]) dargestellt. Eine Auswahl

Tab. 4 Stakeholder-Liste (Ausschnitt)

\begin{tabular}{lll} 
Stakeholder & & \\
\hline Patienten* & einweisende Ärzte* & Personalvertretungen \\
Mitarbeiter* & Versicherer & Staat in diversen Rollen (z.B. als \\
Führungskräfte* & Banken & Ordnungsgeber, Fördermittelgeber, \\
Eigentümer* & andere Erbringer medizinischer & Aufsichtsorgan, Planungsbehörde) \\
Region als Wohn- und Lebensbereich & Leistungen & Lieferanten (Güter und \\
potenzieller Patienten* & Anrainer & Dienstleistungen) \\
Region im Sinne einer Wirtschaftsregion & Zertifizierungsunternehmen & Kammern \\
Krankenkassen* & Selbsthilfegruppen & diverse Verbände \\
Angehörige von Patienten & Politiker & ... \\
Ehrenamtliche Helfer & Kirchen & \\
\hline
\end{tabular}

Die Interessen der mit * gekennzeichneten Stakeholder werden bei den weiteren Ausführungen berücksichtigt. 
der konstitutiven Bedingungen der Leitungsarbeit - die Leitungsstruktur des Krankenhauses und die Organisation betrieblicher Leitungsentscheidungen - werden in ähnlicher Weise in Kapitel 6 thematisiert.

Die Beschreibung der Handlungssituation in den Kapiteln 4.2 bis 4.4 verknüpft die beiden Analyse-Schritte, indem

- die genannten Faktoren im Detail charakterisiert werden und

- anschließend anhand ausgewählter Beispiele gefragt wird, wie Stakeholder möglicherweise reagieren, wenn sich die Handlungssituation des Krankenhauses verändert und die Geschäftsführung mit Entscheidungen, die Folgen für Stakeholder haben können, antworten muss.

\section{Zu 2. Welche Beziehungen gibt es zwischen den Elementen der in Abbildung 21 skizzierten Handlungssituation?}

Das die Handlungssituation des KrankenhausManagements prägende Beziehungs-Modell lässt sich etwa wie folgt charakterisieren (die im Folgenden genannten Ziffern beziehen sich auf die Ziffern in Abbildung 21):

- Veränderungen der die Leitungsarbeit beeinflussenden internen und externen Einflussfaktoren - wie zum Beispiel die Veränderung der Art der Krankenhausleistungs-Vergütung führen nicht selten zu Problemen - wie zum Beispiel zu einer Verknappung des RessourcenEinsatzes (Ziffer 1). Diese werden gelöst, indem Entscheidungen gefällt und die Ergebnisse der Entscheidungen umgesetzt werden (Ziffer 2). Bei der Vorbereitung, dem Fällen und dem Umsetzen dieser Entscheidungen werden die Bedürfnisse der Stakeholder - soweit diese als legitim akzeptiert werden - angemessen berücksichtigt (Ziffer 3).

- Die zu fällenden Entscheidungen haben Folgen:

a) Stakeholder können von den Entscheidungen insofern betroffen sein, als ihre Bedürfnisse nicht berücksichtigt werden und damit ihre eigene Handlungssituation verändert wird - wie zum durch die Kündigung des Arbeitsverhältnisses (Ziffer 4).

b) Stakeholder sind auch indirekt von Veränderungen der die Leitungsarbeit treibenden und/oder restriktiv beeinflussenden Faktoren betroffen, weil die zu fällenden Entscheidungen Veränderungen des Leis- tungsprogramms und/oder der konstitutiven Bedingungen zur Folge haben können (Ziffer 6). Ein neuer Entscheidungsprozess wird ausgelöst (Ziffern 1 und 2); die Bedürfnisse der Stakeholder sind angemessen zu berücksichtigen (Ziffer 3).

- Stakeholder verhalten sich nicht nur passiv. Sie versuchen durch eigene Beiträge proaktiv auf das Geschehen im Krankenhaus Einfluss zu nehmen und verursachen damit Probleme (Ziffer 5), die durch Entscheidungen gelöst werden müssen (Ziffern 1 und 2); die Bedürfnisse der Stakeholder sind angemessen zu berücksichtigen (Ziffer 3) (zu der Rolle der Stakeholder siehe auch die Anmerkungen zu 4.).

- Die für das Krankenhaus typischen Produktions- und kulturellen Bedingungen und externe und interne Einflussfaktoren beeinflussen als Datum die Leitungsarbeit des Krankenhausmanagements (Ziffern 7 und 9). Beide Arten von Faktoren begrenzen möglicherweise den Handlungsspielraum der Verantwortungsträger (Beispiel 2) und damit deren Vermögen, sozial verantwortlich zu handeln.

\section{Beispiel 2}

Die schon mehrfach erwähnte, sich für einzelne Krankenhäuser abzeichnende Notwendigkeit, den Ressourceneinsatz zu reduzieren, verlangt das Heben von Synergieeffekten mittels Abteilungen und Berufgruppen übergreifender Kooperationen. Das für Krankenhäuser typische Bestreben der Abteilungen nach Autonomie und das Bemühen einzelner Berufsgruppen um Emanzipation gegenüber anderen Berufsgruppen schränkt die Handlungsfähigkeit der Geschäftsführung erheblich ein; sie erschweren die Entwicklung der erwähnten Kooperationen und verhindern damit möglicherweise notwendige Effizienzsteigerungen.

- Wenn die krankenhausspezifischen Produktions- und kulturellen Bedingungen durch das Krankenhausmanagement verändert werden (für die kulturellen Bedingungen ist es allerdings umstritten, ob dieses wirklich möglich ist) (Ziffer 8), sind als Folge davon Entscheidungen zu fällen (Ziffern 1 und 2) (Beispiel 3). Die daraus möglicherweise zu erwartenden Konsequenzen für Stakeholder sind dabei angemessen zu berücksichtigen (Ziffer 3). 


\section{Beispiel 3}

Wenn die Geschäftsführung beschließt, die herkömmliche Organisation der Pflege durch eine den Patienten in stärkerem Maße einbeziehende Pflege zu ersetzen, fordert dieses eine Folge-Entscheidung, die Personalausstattung betreffend - mit positiven und negativen Konsequenzen für eine größere Zahl von Mitarbeitern in anderen Leistungsbereichen.

\section{Die Beziehungen zwischen}

- Einflussfaktoren-Veränderungen als Anlass für einen Entscheidungsprozess,

- den Produktions- und kulturellen Bedingungen als eine Art der Bestimmung der Grenzen des Verantwortungsträger-Handlungsspielraums und

- den Folgen für Stakeholder

sind äußerst komplex (s. Abb. 22).

Jede Veränderung von Elementen des Handlungsspielraums kann Folgen für eine Vielzahl von Stakeholdern auslösen. Bevor dieses aber geschehen kann, wird die Entscheidung durch ein möglicherweise breites Spektrum von Bedingungen der Leitungsarbeit gefiltert.

\section{Zu 3. Welchen Zusammenhang gibt es zwischen möglichen Einflussfaktoren-Veränderungen und der Dimension des dem Verantwortungsträger zur Verfügung stehenden Handlungsspielraums?}

Die Beantwortung dieser Frage ist deshalb von zentraler Bedeutung, weil sozial verantwortliches Handeln nur dann möglich ist, wenn dem Verantwortungsträger ein Handlungsspielraum zur Verfügung steht, der es ihm erlaubt, die Befriedigung der Stakeholder-Bedürfnisse in Betracht zu ziehen. Diese Voraussetzung ist nicht immer gegeben:

- Veränderungen der Einflussfaktoren sind einerseits Datum für das Management im Krankenhaus. Sie müssen umgesetzt werden, und zwar unabhängig von den sich daraus für

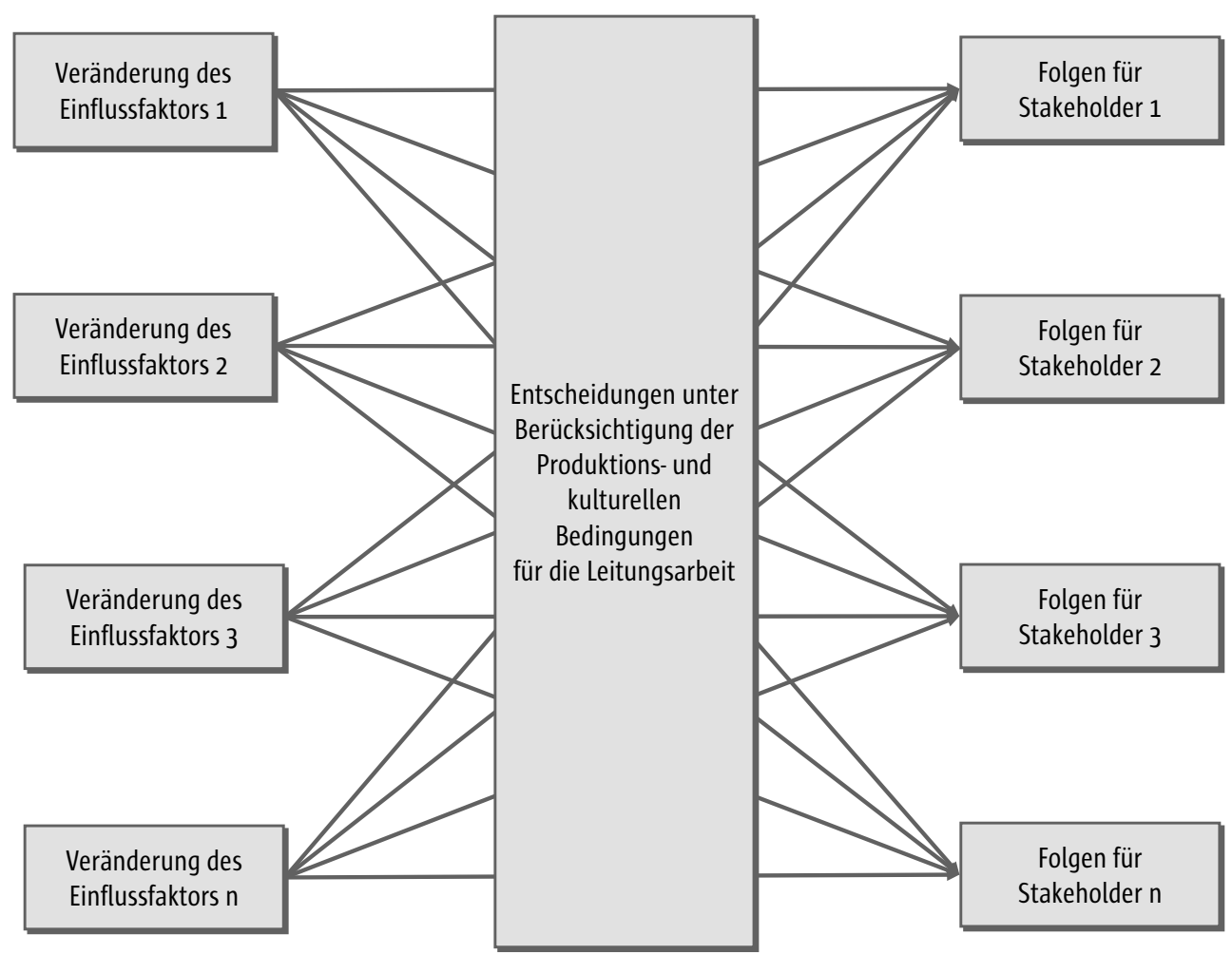

Abb. 22 Zusammenhang zwischen Einflussfaktoren-Veränderungen, Produktions- und kulturellen Bedingungen für die Leitungsarbeit und Folgen für Stakeholder 
Stakeholder ergebenden Folgen (Beispiel 4). Raum für sozial verantwortliches Handeln ist in einem solchen Falle nicht gegeben.

\section{Beispiel 4}

Die Veränderungen finanzieller Rahmenbedingungen - die Umstellung von dem herkömmlichen auf das pauschalierende Entgeltsystem (zu dem DRG-System s. Behrends, S. 7gff.) - müssen von dem Krankenhausmanagement als Datum akzeptiert und entsprechend den gesetzlichen Vorgaben umgesetzt werden.

Die genannten Veränderungen sind nicht nur struktureller, sondern vor allem auch materieller Art: In vielen Krankenhäusern muss deshalb der Ressourceneinsatz reduziert werden. Die Folgen haben vielfach nicht nur die Mitarbeiter zu tragen, weil Arbeitsplätze wegfallen und/ oder Teile der Vergütung (zumindest zeitweise) gestrichen werden und die Arbeitsbelastung für die verbleibenden Mitarbeiter größer wird. Wenn die Reduzierung der Zahl der zur Verfügung stehenden Mitarbeiter nicht durch die Verbesserung der Arbeitsabläufe und durch das Heben von Rationalisierungsreserven ausgeglichen werden kann, sind nicht selten auch die Patienten betroffen.

- Hinsichtlich anderer Einflussfaktoren-Veränderungen sind die Verantwortlichen frei $z u$ entscheiden, ob überhaupt und in welcher inhaltlichen und zeitlichen Ausprägung Veränderungen der Einflussfaktoren bei Entscheidungen berücksichtigt werden (Beispiel 5).

\section{Beispiel 5}

So ist die Geschäftsführung eines Krankenhauses zum Beispiel frei hinsichtlich der Nutzung einer weiterentwickelten Medizin-Technik. Sie kann sich dieser Entwicklung anschließen und in sie investieren; sie kann aber auch darauf verzichten, um Leistungen, die nur auf der Grundlage eines sehr hohen technischen Aufwandes erbracht werden können, von den dafür spezialisierten Leistungserbringern einzukaufen.

Zu den Folgen, die sich einstellen, wenn sich die Geschäftsführung für die Investition in die weiterentwickelte Medizin-Technik entscheidet, siehe ausführlich bei Beispiel 9.

\section{Zu 4. Welche Rollen spielen die Stakeholder?}

Es gibt eine Vielzahl von Personen und Institutionen, denen gegenüber das Krankenhaus verant- wortlich ist und/oder die das Krankenhaus für die Folgen seines Handelns in Anspruch nehmen wollen (s. Tab. 4). Es gibt nicht wenige Stakeholder, die durch eigene Beiträge die weitere Entwicklung des Krankenhauses sichern wollen.

Um im Sinne der Crundidee des Managements der sozialen Verantwortung auf Veränderungen der Einflussfaktoren reagieren zu können, muss der Verantwortungsträger nicht nur wissen, welches die Bedürfnisse der Stakeholder sind. Er muss sich auch im Klaren darüber werden, wie sich Stakeholder in dem Problemlösungsprozess verhalten werden:

- Es fragt sich zum Beispiel, ob die Stakeholder bereit sind, als Ergebnis gezielter Interventionen durch die Geschäftsführung auf die Befriedigung eines Teils ihrer Bedürfnisse $\mathrm{zu}$ verzichten, wenn damit sichergestellt werden kann, dass anderen Anliegen entsprochen werden kann (Beispiel 6).

\section{Beispiel 6}

Die Mitarbeiter erwarten unter anderem die Befriedigung ihres Bedürfnisses nach Sicherheit ihres Arbeitsplatzes und nach einer angemessenen Entlohnung ihrer Tätigkeit. Das Erfüllen dieser Wünsche wird der Geschäftsführung immer wieder erschwert: So müssen in vielen Krankenhäusern als Folge der extern festgelegten Basisfallwert-Reduzierung (zu dem Basisfallwert s. Behrends, S. 79) und der deswegen geringer werdenden Erlöse die Kosten reduziert werden, um Verluste vermeiden zu können.

Immer wieder musste deshalb in der jüngeren Vergangenheit die Geschäftsführung des einen oder anderen Krankenhauses seine Mitarbeiter zu einem Verzicht auf die Befriedigung des einen oder anderen Bedürfnisses zugunsten der Befriedigung anderer Bedürfnisse bewegen. So haben in nicht wenigen Krankenhäusern die Mitarbeiter auf der Grundlage eines zwischen der Geschäftsführung und der Personalvertretung vereinbarten Tarifvertrages über mehrere lahre hinweg auf Teile ihres Urlaubs- und Weihnachtsgeldes verzichtet (betrifft Bedürfnis nach einer angemessenen Entlohnung ihrer Tätigkeit), um damit zur langfristigen Sicherung ihres Arbeitsplatzes (betrifft Bedürfnis nach Sicherheit des Arbeitsplatzes) beitragen zu können (s. zum Beispiel Vivantes, S. 25).

- Stakeholder verhalten sich nicht nur passiv, indem sie die Berücksichtigung ihrer Anliegen durch die Verantwortungsträger erwarten. Auch versuchen sie, durch eigene Beiträge aktiv oder als Reaktion auf eine Entscheidung in 
das Geschehen im Krankenhaus einzugreifen (Ziffer 4 und 5 in Abb. 21). Die Geschäftsführung muss damit rechnen, dass Stakeholder versuchen werden, Entscheidungen proaktiv in ihrem Sinne zu beeinflussen, weil sie

a) mit einer aktuellen Situation nicht einverstanden sind und befürchten, dass die Verantwortungsträger von sich aus nicht tätig werden, und/oder sie

b) die zu erwartenden Folgen der von Verantwortungsträgern zu fällenden Entscheidungen nicht akzeptieren können (Beispiel 7).

\section{Beispiel 7 (s. auch Abb. 23)}

Nicht immer sind die Mitarbeiter - wie in Beispiel 6 angenommen - bereit, die Pläne der Geschäftsführung zu akzeptieren, weil weitreichende Wirkungen für die private Lebensführung der Mitarbeiter die Folgen wären. Die Mitarbeiter werden deshalb rechtzeitig versuchen, die von der Geschäftsführung beabsichtigte Reaktion auf eine Veränderung der finanziellen Rahmenbedingungen abzuwenden.

\section{Zu 5. Welche Beziehungen gibt es zwischen den Zielen, die Stakeholder mittels ihrer Anliegen verfolgen?}

Die Handlungssituation des Krankenhausmanagements zeichnet sich dadurch aus, dass Veränderungen der Handlungssituation und die in deren Folge zu fällenden Entscheidungen mög- licherweise auf jeweils mehrere Stakeholder mit jeweils unterschiedlichen Zielen treffen (s. Abb. 24); die Ziele, die die Stakeholder mit ihren Anliegen verfolgen, können in sehr unterschiedlichen Beziehungen zueinander stehen. Die Verantwortungsträger müssen in die Analyse der Handlungssituation auch diese Frage einbeziehen und gegebenenfalls eine Vor-Entscheidung fällen und damit festlegen, welche Anliegen sie mit welchem Gewicht bei ihren Handlungen berücksichtigen wollen.

Die Ziele sind nicht immer eine feste Größe. Möglicherweise ändern Stakeholder ihre Ziele in Folge einer Entscheidung der Geschäftsführung; so kann es sein, dass sich Stakeholder, die mit ihren Zielen bisher in Konkurrenz zueinander standen, gegen die Geschäftsführung solidarisieren, weil einer der Stakeholder sich als Auswirkung einer unternehmerische Entscheidung als grob benachteiligt wahrnimmt.

Die Identifizierung und Bewertung der verschiedenen Einflüsse und Stakeholder-Anliegen, um sie bei den einschlägigen unternehmerischen Entscheidungen angemessen berücksichtigen zu können, ist deshalb ein höchst komplizierter Prozess.

Praktisch bedeutsam und deshalb für die weitere Untersuchung relevant sind nur a) die ZielAntinomie, b) die Ziel-Konkurrenz und c) die Ziel-Komplementarität: a) die Ziele schließen sich gegenseitig aus, b) die Realisierung eines Zieles schränkt die Realisierung eines anderen Zieles ein

\begin{tabular}{|c|c|}
\hline $\begin{array}{c}\text { Ursache-Wirkungs-Beziehung } \\
\text { - allgemein - }\end{array}$ & $\begin{array}{c}\text { Ursache-Wirkungs-Beziehung } \\
\text { - Beispiel - }\end{array}$ \\
\hline $\begin{array}{c}\text { Veränderung externer } \\
\text { Einflussfaktoren }\end{array}$ & $\begin{array}{c}\text { Veränderung der finanziellen } \\
\text { Rahmenbedingungen }\end{array}$ \\
\hline $\begin{array}{c}\text { Entscheidung der } \\
\text { Geschäftsführung }\end{array}$ & $\begin{array}{c}\text { Reduzierung der Zahl der Stellen } \\
\text { und/oder der Tarife }\end{array}$ \\
\hline $\begin{array}{c}\text { Folgen für den } \\
\text { Entscheidungsspielraum }\end{array}$ & $\uparrow \mid \begin{array}{c}\text { Folgen für die } \\
\text { Lebensführung der } \\
\text { betroffenen Mitarbeiter }\end{array}$ \\
\hline
\end{tabular}

Abb. 23 Wirkung der Einflussfaktoren auf den Entscheidungsspielraum der Stakeholder 


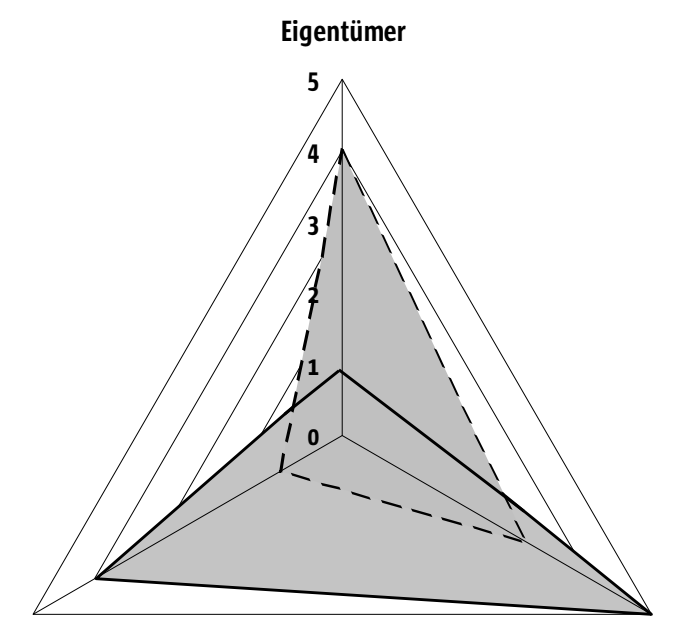

Mitarbeiter

Patienten

Patienten-/Mitarbeiterorientierung $\quad$ I Eigentümerorientierung

Abb. 24 Unterschiedliche Stakeholder-Interessen-Gewichtungen als Grundlage für unternehmerische Entscheidungen

(s. Beispiel 8 und Teile des Beispiels 9) und c) die Realisierung eines Zieles unterstützt die Realisierung eines anderen (siehe Teile des Beispiels 9).

\section{Beispiel 8 (s. Abb. 24)}

Der Krankenhaus-Eigentümer ist neben der Verfolgung auch anderer Ziele an einer angemessenen Verzinsung des von ihm zur Verfügung gestellten Kapitals interessiert. Er erwartet deshalb von der Geschäftsführung, dass diese die Behandlungs- und Supportprozesse so organisiert und/oder dass sie gegebenenfalls die Befriedigung der Bedürfnisse der Mitarbeiter nach Arbeitsplatzsicherheit und einem auskömmlichen Einkommen einschränkt, um einen möglichst hohen Gewinn der Erlöse über die Kosten realisieren zu können.

Wenn die Geschäftsführung dagegen die Interessen der Patienten und die der Mitarbeiter mit einem höherem Gewicht als die Erwartungen des Krankenhauseigentümers berücksichtigt, muss Letzterer seine Erwartungen nach einer Rendite möglicherweise nach unten korrigieren.

\section{Beispiel 9}

Die Geschäftsführung muss akzeptieren, dass sie sich den neuesten medizinischen und technischen Entwicklungen in der Strahlentherapie nicht weiter verschließen kann, und beschließt, eine Abteilung „Strahlentherapie“ aufzubauen. Die dafür notwendigen Investitionen binden Finanzmittel auf längere Zeit in erheblichem Umfang.

Bei seiner Entscheidung musste die Geschäftsführung einerseits davon ausgehen, dass das Krankenhaus die für die Finanzierung der beabsichtigten Investitionen erforderlichen Fördermittel wahrscheinlich nicht erhalten wird; es muss deshalb die Finanzierung gegebenenfalls aus eigenen Rücklagen, die mittels des Erwirtschaftens von Gewinnen gebildet werden konnten, oder zu Lasten der Finanzierung anderer Investitionen sicherstellen. Die Geschäftsführung musste andererseits die weiter unten skizzierten Anliegen mehrerer Stakeholder berücksichtigen. Diese Anliegen konkurrieren miteinander, einige ergänzen sich. Nach dem Abwägen der verschiedenen Interessen hat die Geschäftsführung verfügt, einer kleinen Zahl von (potenziellen) Patienten künftig die für sie erforderliche Therapieform anzubieten; die übrigen Stakeholder können erst später mit der Befriedigung ihrer Bedürfnisse rechnen.

\section{Die Bedürfnisse verschiedener Stakeholder und deren Reaktionen auf die Entscheidung der Geschäftsführung im Einzelnen}

Auf der Grundlage der Investition kann eine kleinere Zahl von Patienten entsprechend den aktuellen Behandlungsleitlinien therapiert werden - was vorher nicht möglich war -, und es können zusätzlich Patienten für die Aufnahme in das Krankenhaus gewonnen werden. Dem Anliegen 
der betroffenen Patienten nach zeitgemäßer Behandlung und dem der Bevölkerung im Einzugsbereich des Krankenhauses nach im Bedarfsfall uneingeschränktem Zugang zu einschlägiger Behandlung wird damit ebenso entsprochen wie dem Bedürfnis einer kleineren Gruppe von Mitarbeitern, die in ihrer Rolle als Agenten der von der Investitionsmaßnahme begünstigten Patienten erwarten, diese künftig bestmöglich behandeln zu können.

Für die dringend notwendige Sanierung von Patientenzimmern stehen in den nächsten Jahren Ressourcen nur in begrenztem Umfang zur Verfügung. Das Bedürfnis aller übrigen im Krankenhaus ambulant und stationär zu behandelnden Patienten nach Verbesserung der Unterbringungsqualität kann einstweilen nur eingeschränkt befriedigt werden. Es muss damit gerechnet werden, dass niedergelassene Ärzte Patienten, welche die neue Therapieform nicht benötigen, nicht mehr in das Krankenhaus einweisen.

Der auf die Verbesserung der Arbeitsbedingungen gerichtete Wunsch der Mitarbeiter, die nicht in der neu geschaffenen Abteilung Strahlentherapie tätig sind, kann vorläufig nur in geringerem Umfang erfüllt werden. Die Leistungsbereitschaft dieser Mitarbeiter wird möglicherweise abnehmen - dieses auch deshalb, weil das ihrer Rolle als Agent der Patienten geschuldete Bedürfnis nach bestmöglicher Behandlung der Patienten - dazu gehört auch die Unterbringung - für die Mehrzahl von Patienten vorläufig nur eingeschränkt befriedigt werden kann.

Die Wirkungen der skizzierten Entwicklungen auf das Verhalten der betroffenen Stakeholder werden verstärkt, weil die erforderlichen Fördermittel von den zuständigen Behörden nicht zur Verfügung gestellt werden und die Investition durch die Reduzierung der Betriebskosten - und das bedeutet: durch die Reduzierung vor allem des Personaleinsatzes - finanziert werden muss. Die Befriedigung der Bedürfnisse eines Teils der Patienten kann also verbessert werden - zu Lasten aber jeweils anderer Stakeholder.

Die Mitarbeiter müssen damit rechnen, dass die der Personalbedarfsermittlung zugrunde liegende Produktivität erhöht und von dem einzelnen Mitarbeiter mehr Leistung erwartet wird. Die Zahl der Stellen wird reduziert; einige Mitarbeiter müssen mit dem Verlust ihres Arbeitsplatzes rechnen. Wenn diese Maßnahmen nicht zu dem gewünschten Erfolg führen, werden möglicherweise zusätzlich Entgelt-Kürzungen für die Mitarbeiter vorgenommen.

Die Krankenkassen müssen damit rechnen, dass - weil andere Fallgruppen in das Leistungsprogramm des Krankenhauses aufgenommen werden - dessen Case-Mix-Index $(\mathrm{CMI})^{32}$ und damit auch das von den Krankenkassen an das

32 Der Case-Mix-Index (CMI) eines Krankenhauses repräsentiert den durchschnittlichen Schweregrad der Erkrankungen der in einem Krankenhaus stationär behandelten Patienten.
Krankenhaus zu zahlende Entgelt höher werden. Sie werden deshalb - weil sie die finanziellen Interessen aller ihrer Mitglieder vertreten müssen - gegebenenfalls versuchen, die Erweiterung des Therapieangebotes, dessen Nutzen nur einigen ihrer Mitglieder zugutekommt und weil die Therapie schon zu dem Leistungsprogramm anderer Krankenhäuser gehört, zu verhindern.

Der Eigentümer des Krankenhauses erwartet, dass das zusätzliche Behandlungsangebot mittel- und langfristig zu einer höheren Inanspruchnahme und damit zu der effizienteren Nutzung der vorgehaltenen Behandlungskapazitäten und damit zu höheren Gewinnen führen wird. Vorübergehend wird er aber seine Renditeerwartungen nach unten korrigieren müssen. Je nachdem, ob der Eigentümer eine mehr langfristig oder eine eher kurzfristig orientierte Strategie verfolgt, wird er die Pläne der Geschäftsführung unterstützen oder versuchen, diese nicht wirksam werden zu lassen.

\subsection{Produktions- und kulturelle Bedingungen der Leitungsarbeit}

\subsubsection{Einführung und Überblick}

Die Produktions- und die kulturellen Bedingungen der Leitungsarbeit in einem Krankenhaus gehören zu den Faktoren, die den Handlungsspielraum des Krankenhausmanagements und damit dessen Möglichkeiten, auf Veränderungen interner und/oder externer Einflussfaktoren im Sinne sozial verantwortlichen Handelns zu reagieren, bestimmen. Dieser Ausschnitt des BeziehungsModells (s. Ziffern 1, 2 und 7 in Abb. 21) kann hier dem zur Verfügung stehenden Platz ist es geschuldet - nicht für alle Arten von Produktions- und kulturellen Bedingungen vollständig thematisiert werden. Zumindest aber anhand eines Beispiels (Näheres s. Kap. 4.2.2, Das Uno-actu-Prinzip) soll der Einfluss dieser Bedingungen auf die Entscheidungsprozesse, die durch Veränderungen interner und/oder externer Einflussfaktoren ausgelöst werden, etwas ausführlicher charakterisiert werden. Ansonsten werden die verschiedenen Bedingungen beschrieben mit Hinweisen einerseits auf Stakeholder-Bedürfnisse, die bei einer vollständigen Analyse der Handlungssituation des Krankenhausmanagements zu berücksichtigen sind, und zum anderen auf eventuelle Einschränkungen der Handlungsspielräume, die von den Bedingungen ausgehen.

Wenn man von Produktion spricht, ist damit im Regelfall ein Prozess gemeint, der aus Aus- 
gangsmaterialien unter Einsatz diverser Produktionsfaktoren (unter anderem der menschlichen Arbeitskraft) ein Endprodukt erzeugt. In diesem allgemeinen Sinne kann auch die Dienstleistung „Herstellung von Gesundheit“ unter den Begriff Produktion subsumiert werden.

Der Transformationsprozess „Produktion“ bedarf eines bestimmten Rahmens - unter anderem einer Leitungsstruktur des Unternehmens und der Gestaltung der Entscheidungsprozesse. In einem Krankenhaus kommen zu diesen Produktionsbedingungen allgemeiner Art spezifische Bedingungen hinzu, die sich aus dem Charakter der dort ausgeübten Dienstleistungstätigkeit ergeben. Diese hat nämlich nicht die Bearbeitung der Ausgangsmaterialien zum Inhalt wie die industrielle oder handwerkliche Produktion oder wie die Dienstleistungstätigkeit „Unternehmensberatung“, sondern die Beschäftigung mit Menschen (Büssing; Glaser, S. 21). Diese werden auf unterschiedliche Weise, und zwar nicht nur als passiv gestaltbares Objekt, in den Produktionsprozess einbezogen und begründen damit die spezifischen Bedingungen der Produktion „Herstellung von Gesundheit“.

Zentrales Element dieser Dienstleistungstätigkeit ist die Interaktion zwischen dem Patienten und den Mitgliedern des therapeutischen Teams. So gesehen, lässt sich der Produktionsprozess in Krankenhäusern anhand der in Tabelle 5 beschriebenen Merkmale kennzeichnen (Büssing; Glaser, S. 21).

Die für die weiteren Überlegungen vor allem relevanten Elemente der Unternehmenskultur sind die den Mitarbeitern gemeinsamen Vorstellungen und Orientierungsmuster. Diese leiten de- ren Handeln, ohne dass sie im Regelfall darüber nachdenken. Dabei handelt es sich um Annahmen unter anderem über menschliche Wesenszüge, über zwischenmenschliche Beziehungen und über Beziehungen zur Umwelt des Unternehmens (Schreyögg; Dabitz, S. zff.). Diese Basisannahmen finden ihren konkreten, für Krankenhäuser typischen Ausdruck in Denk- und Handlungsweisen (s. Tab. 6), die in den folgenden Kapiteln als Grundlage für ihre Berücksichtigung in Entscheidungsprozessen charakterisiert werden.

Die kulturellen Bedingungen für die Leitungsarbeit sind durch die Geschichte des Krankenhauses und durch dessen soziokulturelle Rahmenbedingungen geprägt. Von deren jeweiliger Stärke nämlich von der Klarheit der Vorstellungen und Orientierungsmuster, von der Anzahl der Mitarbeiter, die den Vorstellungen im Arbeitsalltag zur Wirksamkeit verhelfen, und von der Selbstverständlichkeit, mit der Vorstellungen gelebt werden (Schreyögg; Dabitz, S. 5ff.) - hängt es $\mathrm{ab}$, ob die fachlichen, methodischen und sozialen Kompetenzen der Mitarbeiter sowie deren Kreativität und Begeisterungsfähigkeit für die Behandlung der Patienten mobilisiert werden können. Die Stärke kultureller Bedingungen ist bestimmend für die Fähigkeit des Krankenhauses, seine inneren Strukturen zu stärken, und damit für das Schaffen der Voraussetzungen, um auf immer neue Herausforderungen und veränderte Rahmenbedingungen flexibel und sozial verantwortlich reagieren zu können. Von ihr hängt es ab, ob Verbesserungskonzepte begierig aufgenommen und umgesetzt oder ob diese diffamiert und deren Realisierung verhindert werden (Eiff von; Stachel 2007, S. 28).

Tab. 5 Krankenhausspezifische Produktionsbedingungen

Merkmale krankenhausspezifischer Produktionsbedingungen

Bezeichnung Konkretisierung

Uno-actu-Prinzip (s. Kap. 4.2.2)

der Produzent der Leistung und deren Empfänger sind beim Herstellungsprozess gleichzeitig anwesend

Grenzen der Planbarkeit und Unwägbarkeiten als Merkmale der Pflegearbeit (s. Kap. 4.2.3)

die so genannte Ko-Produktionsthese

(s. Kap. 4.2.4) der Pflegeprozess wird unter Berücksichtigung der Reaktionen des Patienten entwickelt - hier wird der Patient als Subjekt gesehen, das passiv auf die von den Mitgliedern des therapeutischen Teams ausgehenden Maßnahmen reagiert

der Patient wird aufgefordert, den Behandlungsprozess aktiv und verantwortlich mit zu gestalten und zu realisieren - die Rolle des Patienten wird als ebenso wichtig für den Behandlungserfolg gesehen wie die der Mitglieder des therapeutischen Teams 
Tab. 6 Elemente einer krankenhausspezifischen Unternehmenskultur

\begin{tabular}{ll} 
Elemente einer krankenhausspezifischen Unternehmenskultur & \\
\hline Bezeichnung & Basisannahmen \\
\hline $\begin{array}{l}\text { Reserviertheit gegenüber der Leitungs- und Organisationsarbeit (s. Kap. 4.2.5) } \\
\text { Autonomie der Leistungsbereiche (s. Kap. 4.2.6) }\end{array}$ & Annahmen über die Natur des Menschen \\
Personenbezogenes Organisationsverständnis (s. Kap. 4.2.7) & Annahmen über die Natur \\
\hline $\begin{array}{l}\text { Notwendigkeit, Interprofessionalität zu organisieren (s. Kap. 4.2.8) } \\
\text { Informelle Prozesse dominieren (s. Kap. 4.2.9) }\end{array}$ & Annahmenmenschlicher Beziehungen \\
\hline Abhängigkeit vom Trägermanagement (s. Kap. 4.2.10) & Anwelt \\
\hline
\end{tabular}

\subsubsection{Das Uno-actu-Prinzip}

\section{Das Konzept}

Eine Besonderheit der Leistungserbringung in einem Krankenhaus ist, dass - von verschiedenen medizinischen und nicht-medizinischen Supportleistungen (wie z.B. von den Leistungen der Laboratoriumsmedizin, der Reinigung und der Wäscheversorgung) abgesehen - der Produzent der Leistung und deren Empfänger bei der Herstellung von Gesundheit gleichzeitig anwesend sind. Die Herstellung der Leistung und deren Konsumption fallen räumlich und zeitlich zusammen. Der Patient ist nicht nur „Objekt“ ärztlicher und pflegerischer Tätigkeiten, sondern Partner im Behandlungsprozess (Büssing; Glaser, S. 21). Das Bedürfnis der Patienten nach persönlicher Zuwendung durch die Mitglieder des therapeutischen Teams wird befriedigt.

Das Uno-actu-Prinzip hat also nicht nur eine technisch-organisatorische, sondern vor allem auch eine - und diese ist für die Analyse der Handlungssituation des Krankenhausmanagements von zentraler Bedeutung - soziale Dimension. Die Interaktion - auch im Sinne von Kommunikation zwischen dem Patienten sowie den Ärzten, Pflegekräften und Vertretern anderer Berufsgruppen, die an der Behandlung des Patienten in der skizzierten Weise unmittelbar beteiligt sind, ist die Kernaufgabe der personenbezogenen Dienstleistung „Patientenbehandlung“ (Büssing; Glaser, S. 21).

\section{Die Praxis}

Der Zusammenhang (s. Abb. 25) zwischen

- der Veränderung eines externen Einflussfaktors,
- dem Handlungsspielraum der Geschäftsführung, der durch die Art des Umgangs mit dem Uno-actu-Prinzip determiniert ist,

- der Berücksichtigung von Patienten-Bedürfnissen bei Entscheidungen, die durch die Einflussfaktoren-Veränderungen ausgelöst werden, und

- eventuellen Reaktionen der Patienten auf die Entscheidung des Verantwortungsträgers wird nachfolgend am Beispiel des durch die Weiterentwicklung der Medizin-Technik ausgelösten Entscheidungsprozesses beschrieben (Näheres zu dem technologischen Wandel s. Kap. 4.3.3).

Angesichts der Weiterentwicklung der Medizin-Technik stellt sich der Geschäftsführung immer wieder die Frage, ob in leistungsfähigere Geräte zwecks Verbesserung der Diagnostik und/ oder der Therapie investiert werden soll (Ziffer 1). Bei der Beantwortung dieser Frage (Ziffer 2) spielt das Uno-actu-Prinzip (Ziffer 7), wenn es denn auch künftig als Basis zur Befriedigung diverser Patienten-Bedürfnisse (Ziffer 3), unter anderem nach persönlicher Zuwendung und Behandlungssicherheit, praktiziert werden soll, eine zentrale Rolle.

Der Einsatz (tele)medizintechnischer Apparate führt unter anderem dazu, dass ,zwischen die mitfühlende Hand des Arztes und den Patienten ... ein bildgebendes Gerät“ (Mannebach, S. 185) geschaltet ist. Der Patient wird aus seiner Rolle als Partner des Behandlungsprozesses teilweise entlassen. Der Charakter der Interaktion zwischen dem Patienten und dem Arzt ändert sich. Die Distanz des Arztes zu menschlicher Krankheit - die er zu seinem Schutz schon immer benötigt - wird durch den Einsatz der medizin-technischen Apparate verstärkt (Danzer, S. 168).

Das gilt umso mehr in Hinblick auf ein Versorgungskonzept, das dank der Entwicklung der 


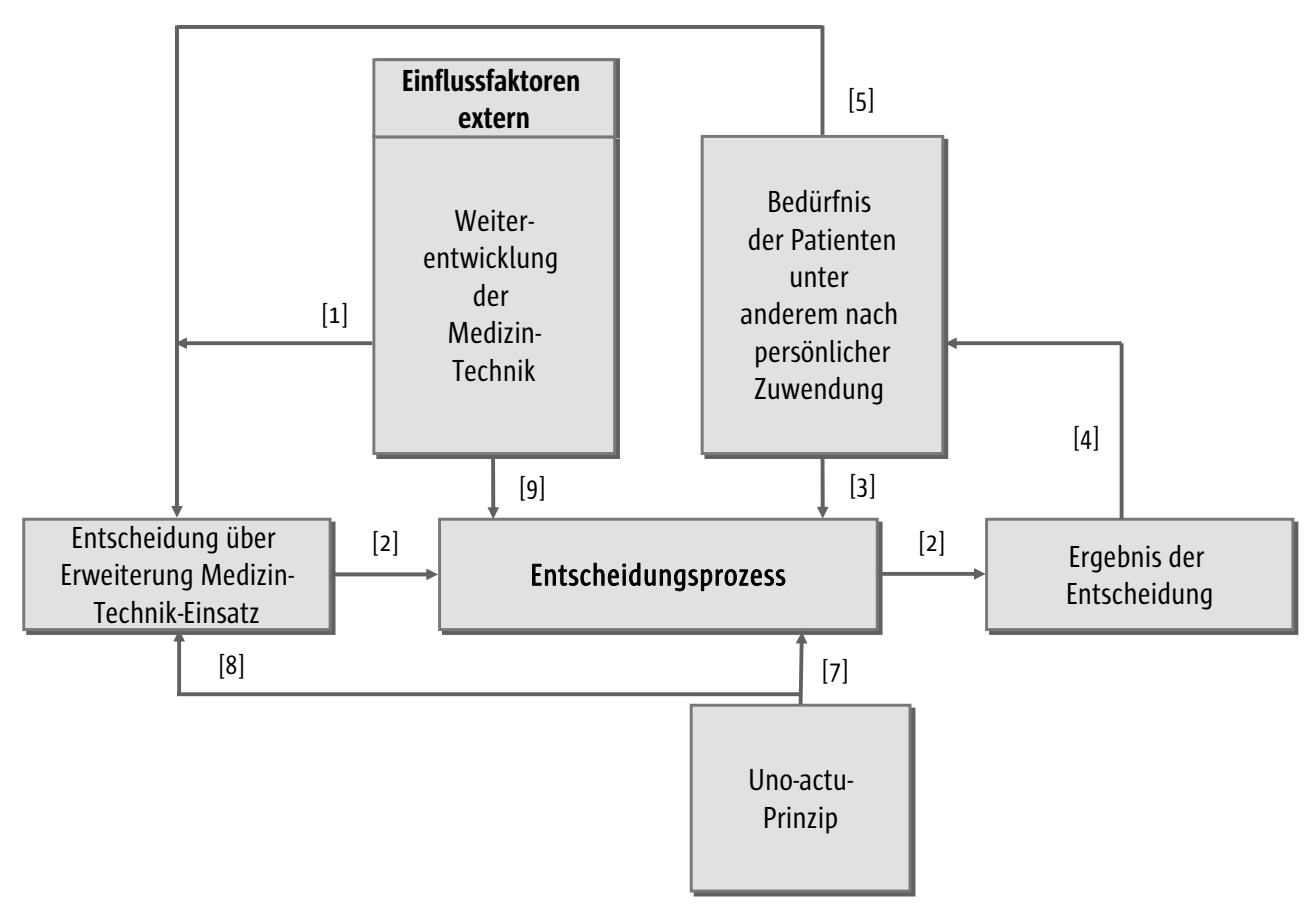

Abb. 25 Diverse Einflussfaktoren und Stakeholder-Anliegen prägen unternehmerische Entscheidungen (s. Abb. 21) am Beispiel Medizin-Technik und Uno-actu-Prinzip

Telemedizin ermöglicht und unter dem Stichwort „Portalklinik“ bekannt geworden ist. Charakteristisch für dieses Konzept ist, dass kleinere Krankenhäuser der Grund- und Regelversorgung eine interdisziplinäre Aufnahmestation mit einer minimalen medizin-technischen Ausstattung vorhalten ${ }^{33}$. Portalkliniken können auf die Beschäftigung von Spezialisten verzichten. Diese stehen vielmehr in einem weiter entfernt verorteten Schwerpunktkrankenhaus zur Verfügung und führen von dort aus für den in die Portalklinik aufgenommenen Patienten - mit Unterstützung der Mitarbeiter der Portalklinik - die Diagnostik durch und legen die erforderliche Therapie fest. Letztere wird - wenn möglich - in der Portalklinik durchgeführt.

Die für die weitere Behandlung so wichtige Interaktion zwischen dem Patienten und dem für die Art der Behandlung ausschlaggebenden Spezialisten findet - wenn überhaupt - ausschließ-

33 Für Portalkliniken wurden verschiedene Konzepte entwickelt und realisiert, die alle dem Grunde nach ähnlich strukturiert sind und funktionieren wie die hier skizzierte. lich mittels Telekommunikation statt. Nur dann, wenn der Patient im Anschluss an die Erst-Untersuchung von der Portalklinik in das Schwerpunktkrankenhaus zwecks weiterer Untersuchungen und/oder anschließender Therapie verlegt wird, kann die für das Erreichen des Behandlungsziels ausschlaggebende Interaktion zwischen dem $\mathrm{Pa}$ tienten und den Mitgliedern des therapeutischen Teams direkt und ohne Einschränkung realisiert werden.

Die Patienten begrüßen einerseits den Einsatz der Medizin-Technik. Sie gewinnen dadurch den Eindruck, mit einem - auch in finanzieller Hinsicht - sehr hohen personellen und technischen Aufwand untersucht und behandelt $z u$ werden. Ihr Bedürfnis nach Wertschätzung (Danzer, S. 166) und Behandlungssicherheit wird befriedigt.

Auf der anderen Seite beklagen die Patienten ihren Objektstatus gegenüber den Apparaten. Sie fühlen sich der Technik ausgeliefert (Halfar, S. 200). Symptome werden auf die Ebene von Maß und Zahl reduziert, personale Aspekte werden aus dem Auge verloren (Danzer, S. 167). Die Patienten vermissen die mitfühlende Hand des Arztes 
und die helfende Hand der Pflegekraft. Die medizin-technischen Geräte werden eher als Barriere zwischen dem Patienten und dem Arzt wahrgenommen. Die Patienten sehen das Crundprinzip einer personenbezogenen Dienstleistung außer Kraft gesetzt. Deren Bedürfnis nach Selbstbestimmung wird nur noch unvollkommen befriedigt. Das Recht auf Autonomie wird eingeschränkt. Der Anspruch der Patienten, als Träger geistigsittlicher Werte um ihrer selbst willen geachtet zu werden, wird durch die Behandlung als „bloßes Objekt" ersetzt.

Die zuletzt zitierte Skepsis vieler Patienten gegenüber (tele)medizinischer Technologie verweist auf Defizite der Kommunikation zwischen Arzt und Patient. Insofern liegt vielleicht der Schluss nahe, dass der medizin-technische Apparat den Arzt nicht zwingen sollte, das Gespräch mit dem Patienten zu vernachlässigen (Halfar, S. 204). Der medizin-technische Apparat muss auch nicht der Grund dafür sein, dass den von ihrer Grundeinstellung her technik-skeptischen Patienten die Befriedigung ihres Bedürfnisses nach Wertschätzung, Behandlungssicherheit, vor allem nach Selbstbestimmung als Ausdruck der Menschenwürde vorenthalten wird.

Wenn der unter bestimmten Voraussetzungen zum Vorteil des Patienten notwendige Einsatz moderner telemedizinischer Technologie nicht durch spezifische Kommunikations-Leistungen des Arztes unterstützt und damit das Uno-actu-Prinzip aktiviert bleibt, muss trotz der zu erwartenden Zustimmung der Patienten zu dem Einsatz der Medizin-Technik mit deren Unzufriedenheit und damit mit Reputationsverlust für das Krankenhaus gerechnet werden. Die Inanspruchnahme des Krankenhauses könnte abnehmen.

Zusammenfassend lässt sich festhalten:

- Die Charakteristik des Uno-actu-Prinzips schränkt den Handlungsspielraum der Verantwortungsträger in Hinblick auf die Nutzung einer weiter entwickelten telemedizinischer Technologie ein.

- Wenn das Uno-actu-Prinzip wegen der die Behandlungs-Qualität stimulierenden Einbeziehung des Patienten in den Behandlungsprozess praktiziert werden soll, dann schließt die Geschäftsführung damit einen intensiven Einsatz der Telemedizin aus - es sei denn, sie investiert nicht nur in die Medizin-Technik, sondern auch in die Verbesserung der Kommunikationsfähigkeiten der Ärzte und ande- rer Mitglieder des therapeutischen Teams. Auf diese Weise können die Barrieren, die durch den Einsatz der Medizin-Technik zwischen Patient und Arzt errichtet werden und die das Uno-actu-Prinzip außer Kraft setzen, überwunden werden.

- Insofern nötigt das Uno-actu-Prinzip die Geschäftsführung in Folge der Entscheidung für den Ausbau der Medizin-Technik zur Lösung eines dadurch neu geschaffenen Problems, das durch die Vernachlässigung diverser Patienten-Bedürfnisse bei der Entscheidung für die Medizin-Technik entstanden ist (s. Abb. 21, Ziffern 4 und 5). Die Lösung des Problems besteht in der einschlägigen Weiterbildung der Mitglieder des therapeutischen Teams.

\subsubsection{Grenzen der Planbarkeit und Unwägbarkeiten als Merkmale der Pflegearbeit ${ }^{34}$}

\section{Das Konzept}

Die Personalbedarfsbestimmung anhand von Kennzahlen, die an Produktivitätsüberlegungen orientiert sind, unterstellt zweckrationales Pflege-Handeln. Dieses kommt darin zum Ausdruck, dass (Böhle; Weishaupt, S. 150)

- alle Arbeitsschritte vorab zeitlich und inhaltlich geplant werden,

- die Arbeitsumgebung exakt und objektiv registriert wird, um sie bei der Ausführung der Arbeitsschritte berücksichtigen zu können, gegebenenfalls auch zur Weiterentwicklung der ursprünglichen Planung, und dass

- der Patient als Objekt wahrgenommen wird, zu dem eine möglichst distanzierte Beziehung gepflegt wird.

Dabei wird übersehen, dass Unbestimmbarkeiten und Unwägbarkeiten zu den bestimmenden Merkmalen der Versorgung und insbesondere der Pflege älterer und alter Patienten gehören (Böhle; Weishaupt, S. 150). Die Bedürfnisse und Befindlichkeiten der zu Pflegenden werden nicht berücksichtigt; Widerstände der zu Pflegenden werden nicht vermieden. Die Pflegearbeit ist wenig sachgerecht und effizient, weil sie zuweilen

34 Die Pflegearbeit steht hier stellvertretend für andere Teil-Prozesse der medizinischen Behandlung. 
auch gegen den Widerstand der zu Pflegenden organisiert und umgesetzt wird.

Als Alternative zu der skizzierten Organisation der Pflegearbeit kommt deshalb „subjektivierendes Arbeitshandeln“ in Betracht (Böhle; Weishaupt, S. 152):

- Auf der Grundlage einer Rahmenplanung wird der Arbeitsablauf der Pflege während der Pflege des Patienten schrittweise entwickelt, und zwar unter Berücksichtigung der Reaktionen des Patienten auf die einzelnen Arbeitsschritte.

- Die Pflege beinhaltet die Offenheit sowohl für ein nicht vollständig vorherseh- und berechenbares Verhalten des Patienten als auch für eine emotionale Beziehung zu diesem als Grundlage für Empathie.

Die Pflegearbeit wird nicht gegen, sondern zusammen mit den zu Pflegenden durchgeführt. Die Qualität und die Effizienz der Pflegearbeit können gesteigert werden. Die Realisierung dieses Konzepts setzt voraus, dass die funktionale Pflege durch ein ganzheitliches Pflegesystem, also die Cruppenpflege ersetzt wird.

Was liegt also näher, als dass die Geschäftsführung angesichts des zunehmenden Alters der Patienten das Konzept des subjektivierenden Arbeitshandelns für die Dienstleistung „Pflege“ ausbaut? Die Geschäftsführung entwickelt für die Akteure der Patientenbehandlung die Handlungsspielräume, die ein den skizzierten Besonderheiten der Patientenbehandlung verpflichtetes Agieren ermöglicht. Sie stellt die finanziellen Mittel bereit, die a) für die Vorbereitung der Führungskräfte und Mitarbeiter auf die Wahrnehmung einer anderen Art von Behandlung und insbesondere von Pflege älterer und alter Patienten und b) für die dauerhafte Realisierung des neuen Pflegekonzepts erforderlich sind. Sie schafft c) die Voraussetzungen, um die emotionale Überforderung der Pflegenden, die in Folge der ständigen und sehr großen Nähe zu den Patienten entstehen könnte, möglichst gering ausfallen zu lassen.

Die Ergebnisse erster Untersuchungen (Böhle; Weishaupt, S. 158ff.) zeigen, dass sowohl die Effizienz und die Qualität der Pflege als auch die Arbeitszufriedenheit der Pflegenden erhöht werden konnten. Andere Untersuchungen (Büssing; Claser, S. 125) kommen zu etwas anderen Ergebnissen. Dort wird festgestellt, dass die emotionale Überforderung der Pflegenden besonders hoch ist, wenn diese sich nicht zwecks Wahrnehmung an- derer Funktionen gelegentlich von „,ihren“ Patienten zurückziehen und damit entlasten können.

Was die Steigerung der Effizienz der Pflegearbeit anbelangt, sind die bisher vorliegenden Untersuchungsergebnisse also noch wenig valide. Es kann deshalb nicht ausgeschlossen werden, dass das Konzept des subjektivierenden Arbeitshandelns dazu führen wird, dass die Kosten des Pflegedienstes - zumindest in der Übergangsphase - steigen werden. Die Umstellung von der Funktions- auf die Gruppenpflege, die Berücksichtigung der Befindlichkeit der zu Pflegenden bei der Realisierung der Pflege und die zur Entlastung der Mitarbeiter erforderlichen „Auszeiten“ sind erste Indizien dafür.

\section{Die Praxis}

Das Krankenhausmanagement muss damit rechnen, dass die personellen Ressourcen künftig nicht mehr in dem Maße wie bisher zur Verfügung stehen werden; der Crund dafür sind Veränderungen der finanziellen und soziodemografischen Bedingungen der Leitungsarbeit. Die Verteilung der Ressourcen auf Leistungsbereiche und innerhalb der Leistungsbereiche auf Kostenarten, das sind unter anderem auch die Berufsgruppen, muss neu justiert werden. Es wird zu Zielsetzungs- und Verteilungskonflikten kommen. Deren Überwindung kann unterstützt werden, wenn die Geschäftsführung die bisher bevorzugte leistungs- bzw. erlösorientierte durch die qualitätsorientierte Personalbedarfsbestimmung (zu den Verfahren der Personalbedarfsbestimmung s. Naegler et al., S. 93ff.) ersetzt.

Trotz der weniger werdenden personellen Ressourcen beschließt die Geschäftsführung, das Konzept des subjektivierenden Arbeitshandelns weiterhin praktizieren zu lassen. Dieses engt allerdings deren Spielraum für Entscheidungen, die zwecks Überwindung der sich abzeichnenden Konflikte gefällt werden müssen, zusätzlich ein. Das ist darauf zurückzuführen, dass die Bedürfnisse einzelner Stakeholder-Gruppen auf der Basis des Konzepts „subjektivierendes Arbeitshandeln“ weiterhin auf einem hohen Niveau befriedigt werden sollen - wie zum Beispiel die der Patienten -, während die Befriedigung der Bedürfnisse anderer Stakeholder-Gruppen - wie zum Beispiel die der Mitarbeiter-Gruppen, die nicht unmittelbar mit der Pflege der Patienten befasst sind umso stärker gekürzt werden muss. Bei diesen 
Entscheidungen muss mit unter anderem folgenden Reaktionen gerechnet werden.

\section{Stakeholder-Anliegen in Bezug auf die Praxis des subjektivierenden Arbeitshandelns}

\section{Entscheidung der Geschäftsführung}

Das Konzept „subjektivierendes Arbeitshandeln“ wird trotz der Verknappung der zur Verfügung stehenden personellen Ressourcen weitergeführt.

\section{Stakeholder und Stakeholder-Anliegen}

\section{Patienten}

Die überwiegende Zahl der Patienten wird die auf der Basis des subjektivierenden Arbeitshandelns praktizierte andere Art des Umgangs mit innen schätzen. Wird doch ihr Bedürfnis nach Selbstbestimmung und Emanzipation gegenüber dem professionellen Urteil der Mitglieder des therapeutischen Teams (Steinkamp; Gordijn, S. 85), nach gefühlsbetonten Kontakten mit anderen Personen und nach Wertschätzung stärker als bisher befriedigt. Zufriedene Patienten werden dazu beitragen, dass die Reputation des Krankenhauses und damit dessen Inanspruchnahme zunehmen.

Es wird sicher aber auch Patienten geben, die mit der innen nun zugeschrieben Rolle - trotz aller Versuche der Pflegenden, ihnen dabei zu helfen, diese neue Rolle zu leben - überfordert sind. Ihr Bedürfnis nach Sicherheit wird nicht ausreichend befriedigt. Sie stehen der eingeleiteten Entwicklung deshalb eher skeptisch gegenüber.

\section{Mitarbeiter}

Die Reaktion der Mitarbeiter wird unterschiedlich ausfallen:

Ärzte (ein Teil von ihnen), Pflegekräfte und die Vertreter anderer Berufsgruppen des therapeutischen Teams werden im Sinne ihrer Agenten-Rolle die Realisierung der Geschäftsführungs-Pläne unterstützen; sie erwarten eine deutliche Verbesserung der Behandlungsqualität. Hinzu kommt, dass auch ihre persönlichen Bedürfnisse nach Sicherheit - im Sinne von Bewältigenkönnen der ihnen als Agent der Patienten übertragenen Aufgaben -, nach gefühlsbetonten Kontakten mit anderen Personen und nach Selbstverwirklichung besser befriedigt werden können.

Die Reputation des Krankenhauses als sozial verantwortlich handelnder Arbeitgeber wird gefördert. Die Fähigkeit des Krankenhauses, auf einem schwieriger werdenden Arbeitsmarkt erfolgreich zu bestehen, wird gestärkt.

Es gibt aber nach wie vor Ärzte, die auf der Grundlage eines weitgehend naturwissenschaftlich ausgerichteten Medizin-Studiums alles ausblenden, was außerhalb dieses naturwissenschaftlichen Paradigmas liegt (Schott, S. 258). Sie sehen den Menschen hinter dem zu behandelnden Fall nicht. Das Konzept des subjektivierenden Arbeitshandelns ist für sie deshalb nicht relevant. Im Gegenteil: Die Realisierung dieses Konzepts steht der Erwartung des Arztes entgegen, dass sich die Sichtweise des Patienten auf seine Krankheit und deren Bedeutung für sein Leben (Steinkamp; Gordijn, S. 85) seinem professionellen Urteil auch weiterhin unterzuordnen habe. Diese Gruppe von Ärzten wird deshalb versuchen, die Realisierung der Geschäftsführungs-Pläne zu verhindern.

Schließlich wird es Mitarbeiter geben, dazu zählen vor allem ältere Pflegekräfte, die das ständige Zusammensein mit den Patienten nicht schätzen werden, weil sie die davon ausgehende emotionale Überforderung fürchten. Sie werden deshalb trotz aller Vorteile, die sie wie die zuerst genannte Gruppe von Mitarbeitern auch sehen, versuchen, die Geschäftsführungs-Pläne nicht wirksam werden zu lassen. Da sie die Möglichkeit dazu wahrscheinlich nicht haben werden, muss mit deren innerer Emigration gerechnet werden.

Mit Widerstand muss auch seitens jener Mitarbeiter gerechnet werden, die von den erwähnten Ressourcen-Verlagerungen unmittelbar oder auch nur mittelbar betroffen sind, weil sie zum Beispiel mit einer Arbeitsverdichtung und damit mit Mehrleistung rechnen müssen.

\section{Eigentümer}

Der Eigentümer wird dem Praktizieren des subjektivierenden Arbeitshandelns eher mit Skepsis begegnen. Zwar begrüßt er den mit der Realisierung dieses Pflege-Konzepts zu erwartenden Reputationsgewinn und die daraus wahrscheinlich resultierende Steigerung der Inanspruchnahme sowie die der Erlöse. Auf der anderen Seite ist noch nicht ausgemacht, ob das subjektivierende Arbeitshandeln nicht auch zu Kostensteigerungen führen wird und wie hoch diese ausfallen werden und ob dadurch der Erlöszuwachs nicht mehr als kompensiert wird.

\subsubsection{Die so genannte Ko-Produktionsthese}

\section{Das Konzept}

In der Praxis und in der Literatur stehen sich zwei unterschiedliche Ausrichtungen von Patientenbehandlung gegenüber (Krenn, Folie 3):

1. Die Patientenbehandlung wird als eine spezifische Art von Erwerbsarbeit angesehen. Diese zeichnet sich dadurch aus, dass ein Objekt bearbeitet wird.

2. Die Patientenbehandlung wird als interaktive Arbeit am Subjekt organisiert. 
Die erste Art der Patientenbehandlung setzt bei dem Bemühen um Erhöhung der BehandlungsEffizienz auf den verstärkten Einsatz der Technik, auf die Standardisierung der Arbeitsabläufe und auf andere Rationalisierungs-Maßnahmen. Als nachteilig wird diese Form der Patientenbehandlung beurteilt, weil die Subjektivität sowohl der Patienten als auch die der Mitglieder des therapeutischen Teams ausgeblendet wird und weil durch Widerstände der Patienten und durch die Förderung der Passivität, Hilflosigkeit und Apathie der Patienten die Behandlungs-Effizienz und -Effektivität leiden.

Die zweite Art der Patientenbehandlung zeichnet sich dadurch aus, dass der Patient in seiner Doppel-Rolle als sowohl Empfänger der Dienstleistung wie auch als Ko-Produzent den Behandlungsprozess und das Ergebnis der Dienstleistung entscheidend prägt. So gesehen ist der Patient nicht einfach Konsument einer Dienstleistung, die in einer ausschließlich biotechnischen Intervention besteht (Badura 1993, S. 29). Er benötigt nicht nur Hilfe, sondern auch der Interaktion mit dem Behandlungsteam als Grundlage für die Entwicklung des Behandlungsziels, für seine Motivation und für das Erlernen von Kompetenzen, um selbst handeln und das Behandlungsziel erreichen zu können (Büssing; Glaser, S. 133). Diese Interaktion ist von unmittelbarer Bedeutung für den Behandlungserfolg im Sinne von Behandlungsqualität und -effizienz und für die Cenesung des Patienten. Die Aufgabe des therapeutischen Teams ist, das Vertrauen des Patienten in die eigene Kraft zu stärken.

Das Uno-actu-Prinzip und das subjektivierende Arbeitshandeln belassen den Patienten weitgehend noch in einer eher passiven Rolle. Es herrscht eine hierarchische Arzt-PatientenBeziehung vor; der Arzt versucht, den Patienten zu motivieren, seine Empfehlungen möglichst umfassend $\mathrm{zu}$ befolgen (= compliance-Konzept). Anders das auf der Ko-Produktionsthese basierende Behandlungskonzept: Von dem Patienten werden Genesungswille und die Bereitschaft, die Entscheidung über die weitere Vorgehensweise gemeinsam mit dem Arzt zu fällen, vorausgesetzt sowie die aktive Mitarbeit bei diagnostischen und therapeutischen Maßnahmen gefordert. Er wird für die Bewältigung seiner Erkrankung verantwortlich gemacht (Badura 1993, S. 29f.). Der Patient muss sich von seiner passiven Konsumentenrolle verabschieden (zu dem Konzept und neueren Forschungsergebnissen der Adherence [= ein anderer Begriff für die Ko-Produktionsthese] siehe Schmacke, Folie 2f. und Härter, Folie 11ff.).

Es muss damit gerechnet werden, dass mancher Patient mit dieser Rolle überfordert sein wird; er wird deshalb das Ko-Produzenten-Konzept nur zögernd annehmen. Es wird aber auch nicht wenige Patienten geben, deren Bedürfnis nach einem sehr viel höheren Maß an Autonomie, nach Selbstbestimmung und Emanzipation gegenüber dem professionellen Urteil der Mitglieder des therapeutischen Teams (Steinkamp; Gordijn, S. 85; Bestmann; Verheyen, S. 20), nach gefühlsbetonten Kontakten mit anderen Personen und nach Wertschätzung auf der Basis dieses Behandlungskonzepts stärker als bisher befriedigt wird. Sie werden feststellen, dass sie mit ihrem aktiven Beitrag zum Gelingen ihrer Behandlung Entscheidendes für die Verbesserung der Behandlungsqualität leisten. So zeigt eine von Janssen-Cilag $\mathrm{GmbH}$ veranlasste und im Mai 2010 durchgeführte Befragung der Bevölkerung in der Bundesrepublik Deutschland, dass etwa $82 \%$ der Befragten es für sehr wichtig halten, in die Planung ihrer Behandlung eingebunden zu werden. Etwa 60\% der Befragten sind bereit, mit dem Arzt gemeinsam verbindliche Zielvereinbarungen zu treffen, ihre Behandlung betreffend.

Die Integration des Patienten in diese Art von Behandlungsprozess ist gebunden an spezifische Fähigkeiten von Ärzten, Pflegekräften und Vertretern anderer Berufsgruppen, insbesondere an deren Bereitschaft zur Abkehr von einer berufsständisch orientierten hin zu einer an dem mit dem Patienten vereinbarten Behandlungsziel ausgerichteten Zuordnung von Aufgaben und die Fähigkeit, über die medizinische Intervention i.e.S. hinaus den Patienten im Sinne des neuen Behandlungskonzepts gewissermaßen zu coachen.

Es muss damit gerechnet werden, dass der personelle Aufwand höher sein wird als bei der Realisierung konventioneller Behandlungs-Konzepte.

\section{Die Praxis}

Nun ist aber die Organisation Krankenhaus geprägt durch das Neben- und Miteinander unterschiedlicher professioneller Traditionen und Kulturen (Grossmann, S. 305). Das Bestreben der Berufsgruppen nach Arbeitsteilung sowie das Bemühen um fachliche Abgrenzung und das Stärken der eigenen Identität bestimmen den Arbeitsall- 
tag. Die Geschäftsführung muss - wenn sie dem auf der Ko-Produktionsthese aufbauenden Behandlungskonzept zur flächendeckenden und dauerhaften Wirksamkeit verhelfen will - deshalb damit rechnen, dass dem die skizzierten kulturellen Bedingungen entgegenstehen.

Damit die Patienten und die Mitarbeiter, die im Sinne ihrer Agenten-Rolle dennoch dem neuen Behandlungskonzept den Vorzug geben möchten, auf die Realisierung der weiter oben skizzierten Vorteile des neuen Behandlungskonzepts nicht verzichten müssen, muss die Geschäftsführung versuchen, ihren Handlungsspielraum im Sinne der Realisierung des durch Ko-Produktionsthese gestützten Behandlungskonzepts zu erweitern. Durch geeignete Integrationsleistungen versucht sie, Interprofessionalität zu organisieren, um dadurch die zu erwartenden Widerstände überwinden zu können

Trotz der zu erwartenden Ressourcen-Verknappung beschließt die Geschäftsführung, das auf der Ko-Produktionsthese aufbauende Behandlungs-Konzept weiterhin praktizieren zu lassen. Dieses engt allerdings deren Spielraum für Entscheidungen, die zwecks Überwindung der sich abzeichnenden Konflikte gefällt werden müssen, zusätzlich ein. Das ist darauf zurückzuführen, dass die Bedürfnisse einzelner StakeholderGruppen weiterhin auf einem hohen Niveau befriedigt werden sollen - wie zum Beispiel die der Patienten -, während die Befriedigung der Bedürfnisse anderer Stakeholder-Cruppen - wie zum Beispiel die der Mitarbeiter-Cruppen, die nicht unmittelbar mit der Pflege der Patienten befasst sind - umso stärker gekürzt werden muss. Die Geschäftsführung muss deshalb damit rechnen, dass ähnliche Reaktionen ausgelöst werden wie sie im Zusammenhang mit der Umsetzung des Behandlungs-Konzepts „,subjektivierendes Arbeitshandeln" beschrieben wurden.

\subsubsection{Reserviertheit gegenüber Leitungs- und Organisationsarbeit ${ }^{35}$}

Für den Expertenbetrieb Krankenhaus - andere Expertenbetriebe sind unter anderem Schulen, Hochschulen, Orchester und Theater - ist charakteristisch, dass sich Ärzte, Pflegekräfte und

35 Die Ausführungen in den Kapiteln 4.2.5 bis 4.2.10 in Anlehnung an Grossmann 1993, S. 304ff. medizin-technisches Personal primär den Inhalten ihrer Arbeit verpflichtet sehen; dazu zählt auch das Entwickeln der Arbeitsbedingungen innerhalb ihres Leistungsbereiches. Das Gestalten der Schnittstellen zu anderen Leistungsbereichen und damit der Organisation des Krankenhauses als Ganzes erwarten sie gewissermaßen als Service des Krankenhauses; die Experten halten derartige Tätigkeiten für Zusatzarbeiten, die sie von ihrer eigentlichen Profession, der Behandlung der Patienten und/oder der Forschung, ablenken. Die notwendige Organisationsarbeit wird deshalb von Stellen der Verwaltung geleistet, was nicht selten zu erheblichen Spannungen zwischen den Vertretern des ärztlich-pflegerischen Dienstes und den Instanzen der Verwaltung führt; die Akzeptanz der Ergebnisse der Organisationsarbeit lässt zu wünschen übrig.

Bei der Besetzung von Leitungspositionen im Ärztlichen Dienst wird immer noch nicht durchgängig Wert auf eine einschlägige Leitungsqualifikation gelegt. Die Chefarztposition wird nicht selten mit einem Arzt besetzt, der vor allem durch seine hervorragenden Fähigkeiten als Chirurg oder als Internist ausgewiesen ist. Entsprechend reserviert sind die leitenden Ärzte gegenüber der Wahrnehmung der Aufgabe als Führungskraft eines Leistungsbereichs mit häufig einer großen Zahl von Mitarbeitern und einem entsprechend umfangreichen Budget. Sie haben Schwierigkeiten zu akzeptieren, dass Führungsleistungen als Interaktion zwischen der Führungskraft und dem Mitarbeiter durch die Führungskraft persönlich erbracht werden müssen und dass die Mobilisierung der Mitarbeiter zur Übernahme von Verantwortung und zu einem Hierarchieebenen, Berufgruppen und Leistungsbereiche übergreifenden Dialog nicht, und schon gar nicht an die Personalabteilung, delegiert werden kann.

Die Geschäftsführung vieler Krankenhäuser ist in den letzten Jahren dazu übergegangen, den Führungskräften in der zweiten Leitungsebene neben der Verantwortung für die Qualität der Patientenbehandlung auch die für das finanzielle Ergebnis ihres Leistungsbereichs zu übertragen. Das hat dazu geführt, dass das Interesse der Führungskräfte an dem Erwerb bzw. an dem Ausbau der Leitungs- und Organisationskompetenz sowie an deren Umsetzung in praktisches Handeln zugenommen hat. Wichtig wäre nun, dass die Geschäftsführung diesen Wandel unterstützt und mehr in diese Qualifikation ihrer leitenden Mitarbeiter investiert. 
Diese Weiterentwicklung der Führungskräfte ist notwendig, damit die Geschäftsführung in der ihr nachgeordneten Leitungsebene die Partner hat, die helfen können, Entscheidungen, die in Folge der skizzierten Herausforderungen und der Veränderungen der Rahmenbedingungen - wie zum Beispiel die Umstellung des bisherigen auf ein pauschalierendes Entgeltsystem - gefällt werden müssen, im Sinne der Crundidee des Managements der sozialen Verantwortung vorzubereiten und umzusetzen, oder die diese Leitungstätigkeit in eigener Verantwortung realisieren.

\subsubsection{Autonomie der Leistungsbereiche}

Eine Stärke des Krankenhauses und eine Schwäche zugleich ist das Bestreben seiner Leistungsbereiche nach Autonomie, und zwar Autonomie im Sinne einer möglichst geringen Einschränkung des Entscheidungs- und Handlungsspielraums. Die Leistungsbereiche verfolgen ihre eigenen professionellen Interessen, vor allem was die Verfügbarkeit über Ressourcen anbelangt - und dies nicht selten zu Lasten anderer Organisationseinheiten. Die Akteure konzentrieren sich auf die Lösung bereichsinterner Probleme und vernachlässigen die Verzahnung mit anderen, an der Behandlung ,ihrer“ Patienten beteiligten Leistungsbereiche (Schaeffer, S. 270). Die medizinischen Leistungsbereiche sind nach außen (= außerhalb des Krankenhauses) orientiert und pflegen intensiv ihre Beziehungen zu Fachkollegen und Institutionen unterschiedlicher Art; sie leisten damit einen nicht zu unterschätzenden Beitrag zur Weiterentwicklung ihres Faches und zur Professionalisierung ihrer Mitarbeiter.

Auch in diesem Zusammenhang wird von der Geschäftsführung ein hohes Maß an Integrationsleistung gefordert. Es gilt, die - aus der Sicht der Patienten, aber auch aus der Sicht des Unternehmens insgesamt - „organisierte Unverantwortlichkeit“" (Beck, S. 96ff.) mit negativen Folgen für die Qualität und Effizienz der Patientenbehandlung und für die langfristige finanzielle Sicherung des Unternehmens zu überwinden. Dabei steht die Geschäftsführung vor einem nur schwer zu lösenden Dilemma: Auf der einen Seite muss sie dafür sorgen, dass die einzelnen Schritte der Patientenbehandlung, an der im Regelfall mehrere Leistungsbereiche beteiligt sind, besser als bisher aufeinander abgestimmt werden. Um dieses erreichen zu können, wird sie nicht umhin kommen, die Leistungsbereiche zur Kooperation zu verpflichten und damit die Autonomie der Leistungsbereiche einzuschränken. Auf der anderen Seite ist mehr Autonomie ein Zeichen für das Vertrauen, das die Geschäftsführung den Leistungsbereichen entgegenbringt; die Fähigkeit der Mitglieder der Leistungsbereiche, verantwortungsvoll zu handeln, und die Fähigkeit des Krankenhauses, auf die skizzierten Herausforderungen und die Veränderungen der Rahmenbedingungen erfolgreich zu reagieren, werden gestärkt.

Ein Anlass, sich mit der Frage nach dem wünschenswerten Grad der Autonomie der Leistungsbereiche auseinanderzusetzen, ist die Umstellung der Krankenhausfinanzierung auf das pauschalierende Entgeltsystem. Diese hat dazu geführt, dass die Krankenhäuser ein großes Interesse daran haben, die Dauer der Behandlung auf das medizinisch notwendige Maß abzukürzen. Der organisatorischen Integration, der Konzentration auf die Wahrnehmung von Aufgaben, die Leistungsbereiche übergreifen, und der Entwicklung der Gesamtorganisation wird jetzt sehr viel mehr Aufmerksamkeit gewidmet.

\subsubsection{Personenbezogenes Organisations- verständnis}

An der Spitze von Krankenhäusern und vor allem der klinischen Abteilungen standen und stehen noch immer charismatische Persönlichkeiten, deren herausragende individuelle Eigenschaften und Ausstrahlungskraft, verbunden mit einem beachtlichen Motivationseffekt, häufig mit Leitungskompetenz gleichgesetzt werden. Auf diese Persönlichkeiten sind nicht selten die Leitungsstruktur und die Besetzung von Leitungspositionen zugeschnitten. Die Defizite, die in den Kapiteln 4.2.5 und 4.2 .6 beschrieben werden, werden durch diese Art von personenbezogenem Organisationsverständnis verstärkt.

Ein professionelles Verständnis für eine mehr an den wahrzunehmenden Aufgaben orientierte Gestaltung der Leitungsstruktur und der Arbeitsabläufe fehlt. Die Führungsarbeit ist durch Elemente eines eher autoritären Führungsstils charakterisiert. Fehler werden vor allem der Führungskraft zugerechnet, was verhindert, dass eine sachorientierte Fehleranalyse durchgeführt wird.

Wenn die das Geschehen im Krankenhaus prägende Persönlichkeit ausscheidet, entsteht ein 
Leitungsvakuum, das zu ernstlichen Beeinträchtigungen hinsichtlich der Qualität und der Effizienz der Behandlungs-, Support- und Betriebsführungsprozesse führen kann. Es bedarf großer Anstrengungen, bis die strukturellen und organisatorischen Defizite aufgearbeitet worden sind.

Der Handlungsspielraum der Geschäftsführung wird mitunter entscheidend eingeschränkt, weil ihr in der ihr nachgeordneten Leitungsebene die Partner fehlen, deren Unterstützung sie zur Wahrnehmung von Verantwortung benötigt.

\subsubsection{Notwendigkeit, Interprofessionalität zu organisieren}

Die Organisation Krankenhaus ist geprägt durch das Neben- und Miteinander unterschiedlicher professioneller Traditionen und Kulturen (Grossmann, S. 305). Fachliche Abgrenzung und Arbeitsteilung und notwendige Kooperationen über die Berufsgruppen hinweg sowie ein hohes Maß von Abhängigkeiten der verschiedenen Berufsgruppen voneinander ist charakteristisch für die Krankenhaus-Routine. Dieser Zustand wird überlagert durch das Bemühen einzelner Berufsgruppen, vor allem aber einzelner ihrer Repräsentanten, um Emanzipation und Identität sowie um die Verankerung in den Leitungsstrukturen. Sehr viel Kraft entgeht dadurch der Wahrnehmung der Kernaufgaben des Krankenhauses.

Um die strukturell bedingten Spannungen überwinden zu können, verlangt Leitungsarbeit in einem Krankenhaus ein hohes Maß an Integrationsleistungen durch die Geschäftsführung, gegebenenfalls auch durch die für das Krankenhaus zuständigen Aufsichtsorgane. Dieser Anforderung gerecht $\mathrm{zu}$ werden, wird allerdings in vielen Krankenhäusern derzeit noch dadurch erschwert, dass diese durch ein Kollegialorgan geleitet werden, das im Regelfall mit einem Arzt, einer Krankenpflegekraft und einem Ökonomen besetzt ist und sich hinsichtlich des Realisierens der verlangten Integrationsleistungen selbst im Wege steht und als Vorbild im Sinne des Managements der sozialen Verantwortung nicht taugt.

Das Kollegialorgan hat den Auftrag, das Krankenhaus gemeinsam zu leiten. Nicht selten werden Entscheidungen aber auch dieses Kollegiums durch die Interessen einzelner Berufsgruppen dominiert. Diese Anliegen werden häufig nicht explizit in den Entscheidungsprozess eingeführt, sodass eine Verständigung im Dialog über die Le- gitimität und die Gewichtung der verschiedenen Anliegen nicht möglich ist. Eine der Grundideen des Managements der sozialen Verantwortung ist damit außer Kraft gesetzt - im Dialog herauszufinden, welche Stakeholder durch unternehmerische Entscheidungen auf welche Weise betroffen sind, um diese Informationen bei den Entscheidungen berücksichtigen zu können.

Zunehmend werden Führungskonzepte entwickelt, implementiert und gelebt, die dem Bemühen um Abbau der Spannungen zwischen den Berufsgruppen Rechnung tragen:

- Die Führungsorganisation wird im Sinne dezentraler Führung gestaltet.

- Mitarbeiter werden auf der Grundlage einer partizipativen Führung in die Entscheidungsprozesse einbezogen.

- Das Postulat der Individualisierung anstatt kollektiver Regelungen gewinnt an Bedeutung. Damit wird ein Ausgleich zwischen

- den Interessen der Vertreter verschiedener Berufsgruppen - vor allem der Ärzte und der Pflegekräfte - nach jeweils möglichst hoher Gewichtung berufsspezifischer Anliegen bei unternehmerischen Entscheidungen und

- den Anliegen jener Leitungskräfte, die bei der Leitung einzelner Leistungsbereiche und/oder des Krankenhauses die Integration der unterschiedlichen professionellen Interessen bevorzugen, möglich.

\subsubsection{Informelle Prozesse dominieren}

Jedes multipersonal und arbeitsteilig organisierte Unternehmen benötigt Normen, in denen festgelegt ist, wer welche Aufgaben zu erfüllen, wer welche betriebliche Leitungsentscheidungen nach welchen Regeln zu fällen und wer das Ergebnis der Entscheidung umzusetzen hat. In einer Parallelwelt zu dieser formalen Ordnung gibt es informelle Prozesse. Auch in dieser Welt werden Entscheidungen gefällt und umgesetzt, die allerdings im Regelfall durch nichts legitimiert sind und die einen Beitrag zu der gezielten Weiterentwicklung des Unternehmens nur in Ausnahmefällen und die formellen Prozesse eher nur unterstützend leisten können.

Informelle Prozesse können eine Stärke der Organisation sein, sie sind immer auch deren Schwäche: 
- Informelle Prozesse können einerseits zur Funktionsfähigkeit der Organisation beitragen. Sie werden zur Vorbereitung von Entscheidungen genutzt, um die bei der Entscheidung zu berücksichtigenden Anliegen der verschiedenen Stakeholder identifizieren, bewerten und berücksichtigen zu können, um sich über die Umsetzungsbedingungen zu informieren und um die Akzeptanz der Umsetzung des Entscheidungsergebnisses sichern zu können. Der Handlungsspielraum der Verantwortungsträger kann dadurch erweitert werden. Der Beitrag informeller Prozesse zur Funktionsfähigkeit der Organisation ist allerdings im Regelfall ein eingeschränkter. Nicht selten erfolgt die Analyse der zu berücksichtigenden Stakeholder-Interessen eher selektiv; es werden die Anliegen möglicherweise nur der Stakeholder in dem Entscheidungsprozess berücksichtigt, die sich der besonderen Gunst des Entscheidungsträgers erfreuen. Die anhand der Analyse erhobenen Informationen werden nicht immer explizit in einen Entscheidungsprozess eingeführt.

- Zum anderen werden die im Rahmen der formalen Ordnung getroffenen Entscheidungen auf der informellen Ebene unterlaufen - nicht selten, weil Stakeholder-Interessen nicht im Sinne jener als legitim ausgewiesenen Stakeholder berücksichtigt worden sind. Immer wieder wird auf der informellen Ebene im Widerspruch zu der Entscheidung, die durch die formell dazu befugte Instanz getroffen wurde, versucht, das, was ein einzelner Stakeholder will, durchzusetzen und anderen aufzuoktroyieren. Intransparenz des Leitungsgeschehens und dadurch entstehende Gerüchte begünstigen diese Entwicklungen. Insofern stützen informelle Prozesse die Verstärkung der Schwerfälligkeit sozialer Systeme und erschweren die gezielte Weiterentwicklung und Anpassung des Krankenhauses an neue Herausforderungen und sich ändernde Rahmenbedingungen.

Die Dominanz informeller Prozesse trägt dazu bei, dass eine Reihe von Crundpostulaten des Managements der sozialen Verantwortung (s. Kap. 6.2) nicht realisiert wird - mit den schon skizzierten Folgen. Vor allem besteht die Gefahr, dass nur die Bedürfnisse ausgewählter und nicht aller als legitim charakterisierter Stakeholder berücksichtigt werden. Entscheidungen werden nur ausnahms- weise auf der Basis einer argumentativen, dialogischen Verständigung gefällt. Der Pflicht, das Ergebnis einer Entscheidung zu begründen, kommt man vielfach nicht nach.

Informelle Prozesse wird man nicht gänzlich unterdrücken können. Die Geschäftsführung ist aber aufgerufen, Maßnahmen für das Schaffen einer inneren Struktur des Krankenhauses als Basis für verantwortliches Handeln, für die institutionelle Verankerung des Managements der sozialen Verantwortung in der Krankenhausorganisation (Einzelheiten dazu s. Kap. 7) zu planen und umzusetzen, um die Wirkungen informeller Prozesse auf den eher unterstützenden Teil zu begrenzen.

\subsubsection{Abhängigkeit vom Trägermanagement}

Die Verteilung der Leitungs-Kompetenzen auf Instanzen des Krankenhaus-Trägers einerseits und auf die der Geschäftsführung auf der anderen Seite ist oft unscharf. Fehlentwicklungen sozialer und/oderökonomischer Art können jeweils der anderen Seite zugeordnet werden. Sachfremde Argumente beeinflussen häufig selbst die Entscheidungen auf der operativen Ebene.

Entscheidend aber für die Schwäche einer solchen Organisation ist, dass der skizzierte $\mathrm{Zu}$ stand - und das gilt vor allem für nicht wenige Krankenhäuser in öffentlicher und in frei-gemeinnütziger Trägerschaft - über Jahrzehnte hinweg akzeptiert worden ist. Das Krankenhaus hat vielfach nicht die Chance gehabt, sich zu emanzipieren und zu einer lernenden Organisation zu entwickeln. Es kann die immer wieder neuen Herausforderungen und die sich ändernden Rahmenbedingungen nicht erfolgreich bewältigen und ist dem Wettbewerb nicht mehr gewachsen. Es dauert dann nicht mehr lange, dass - was vor allem im öffentlichen Bereich zu beobachten ist das Krankenhaus fallen gelassen wird.

Die Verantwortlichen beim Krankenhausträger verunmöglichen auf diese Weise die Befriedigung einer ganzen Reihe von Stakeholder-Anliegen:

- Der Handlungsspielraum der Geschäftsführung und anderer Verantwortungsträger im Krankenhaus und damit das Wahrnehmen von Verantwortung werden eingeschränkt.

- Das Bedürfnis der Geschäftsführung nach Selbstverwirklichung, Selbstentfaltung und Eigenständigkeit, nach partizipativer Füh- 
rung durch die vorgesetzte Instanz beim Krankenhausträger wird nicht befriedigt.

- Das gilt auch hinsichtlich der Interessen der Mitarbeiter. Wenn das Trägermanagement nicht nur die strategischen, sondern auch operative Entscheidungen fällt, wird damit nicht nur der Handlungsspielraum der leitenden, sondern auch jener der nachgeordneten Mitarbeiter mehr oder weniger deutlich eingeengt. Die Bereitschaft zu besonderen Anstrengungen kann damit nicht beflügelt werden. Bedürfnisse vieler Stakeholder können bei unternehmerischen Entscheidungen nicht berücksichtigt werden, weil die Entscheidungsträger diese Bedürfnisse nicht kennen.

- Das Trägermanagement riskiert mit seinem Verhalten, dass das Krankenhaus entweder verkauft werden muss oder vom Markt verschwindet mit unkalkulierbaren Folgen unter anderem für

- die Menschen im Einzugsbereich des Krankenhauses, die im Bedarfsfall die schnelle medizinische Hilfe in ihrer Nachbarschaft nicht mehr finden,

-1. die Mitarbeiter, die ihren Arbeitsplatz verlieren mit Konsequenzen für ihre private Lebensführung, und

- die Lieferanten in der Nachbarschaft des Krankenhauses, die Umsatzeinbußen hinnehmen und gegebenenfalls Arbeitsplätze abbauen müssen.

Eine derartige Entwicklung kann nur dann vermieden werden, wenn auch der KrankenhausTräger zu einem Paradigmen-Wechsel bereit ist und verantwortliches Handeln im Sinne des Managements der sozialen Verantwortung auf allen Leitungs-Ebenen und von allen Mitarbeitern sowohl in den Instanzen des Krankenhaus-Trägers, soweit sie dort für das Krankenhaus verantwortlich sind, als auch im Krankenhaus einfordert und ermöglicht.

\subsection{Herausforderungen für die Geschäftsführung}

\subsection{1 Überblick}

Die Herausforderungen, mit denen sich die Geschäftsführung eines Krankenhauses immer wieder konfrontiert sieht, sind in der Tabelle 7 im Überblick und nicht mit dem Anspruch auf Vollständigkeit dargestellt.
Tab. 7 Herausforderungen für die Geschäftsführung

Die Leitungsarbeit treibenden Faktoren

Veränderungen der Inzidenz und der Prävalenz von Krankheiten (s. Kap. 4.3.2),

Weiterentwicklung der Medizin-Technik und der Informations- und Kommunikationstechnologie (s. Kap. 4.3.3),

Veränderungen in der Organisation des Gesundheitssystems und der Leistungserbringer (s. Kap. 4.3.4),

Veränderungen des in dem Krankenhaus gelebten Wertesystems (s. Kap. 4.3.5) sowie

Veränderungen der Struktur des Krankenhaus-,,Marktes“ (s. Kap. 4.3.6).

\subsubsection{Veränderungen der Inzidenz und der Prävalenz von Krankheiten}

In den letzten Jahren konnte beobachtet werden, dass sowohl die Inzidenz als auch die Prävalenz die Prävalenz ist das Produkt aus Inzidenz, also aus der Zahl der neu aufgetretenen Krankheitsfälle, und der durchschnittlichen Krankheitsdauer (Niehoff, S. 36) - bestimmter Krankheiten zugenommen haben; zu diesen Krankheiten zählen zum Beispiel der Diabetes oder die Erkrankung an AIDS. Dagegen hat die Zahl der Neuerkrankungen zum Beispiel bei Schlaganfällen abgenommen. Die Geschäftsführung muss sich darauf einstellen, das Leistungsprogramm der sich gegebenenfalls ändernden Nachfrage anzupassen. Sie muss in neue Behandlungskapazitäten investieren und/oder andere Behandlungskapazitäten reduzieren. Dabei sollten die im Folgenden dargestellten Stakeholder-Anliegen berücksichtigt werden. Es muss mit den dort skizzierten Reaktionen gerechnet werden.

\section{Stakeholder und deren Anliegen, bezogen auf Veränderungen der Inzidenz und Prävalenz}

\section{Entscheidung der Geschäftsführung}

Das Leistungsangebot sowie die dafür notwendigen Behandlungskapazitäten werden der sich ändernden Nachfrage angepasst.

\section{Stakeholder und Stakeholder-Anliegen}

\section{Patienten}

Die Veränderungen der Inzidenz und der Prävalenz von Krankheiten und die dadurch ausgelösten Veränderungen 
der Behandlungskapazitäten werden von den Patienten, die bereits in die stationäre Behandlung aufgenommen worden sind, - anders als von den einweisenden Ärzten und der Bevölkerung im Einzugsbereich des Krankenhauses - nicht wahrgenommen. Deren Bedürfnis nach angemessener Behandlung wird nicht tangiert. Sie werden sich zu den Plänen der Geschäftsführung deshalb nicht äußern.

\section{Bevölkerung im Einzugsbereich des Krankenhauses und niedergelassene Ärzte}

Spätestens dann, wenn ein Mensch mit Verdacht auf Behandlungsbedürftigkeit nicht zeitgerecht in die stationäre Behandlung in dem von ihm präferierten Krankenhaus aufgenommen werden kann, weil einschlägige Behandlungskapazitäten fehlen, wird das Verlangen nach Erweiterung der Behandlungskapazitäten sowohl von der Bevölkerung im Einzugsbereich des Krankenhauses wie auch von den niedergelassenen Ärzten, die mit dem Krankenhaus zusammenarbeiten, artikuliert.

Wenn die Geschäftsführung plant, nicht mehr erforderliche Kapazitäten zu reduzieren, muss sie mit erheblichen Widerständen durch die Bevölkerung im Einzugsbereich und durch die niedergelassenen Ärzte rechnen; die Einwohner im Einzugsbereich befürchten, künftig im Bedarfsfall nicht mehr zeitgerecht aufgenommen werden zu können. Diese Widerstände sind im Regelfall mit einer asymmetrischen Informationsverteilung begründet. Sie können durch die sach- und zeitgerechte Information der vermeintlich Betroffenen vermieden oder überwunden werden.

\section{Mitarbeiter}

In der Folge von Kapazitätsanpassungen wird die Zahl der Stellen für Mitarbeiter neu festgelegt. In der Praxis bedeutet dieses nicht selten, dass bei einer Reduzierung der Behandlungskapazitäten die Zahl der Stellen für Mitarbeiter proportional zu der Veränderung der Kapazitäten angepasst werden; hingegen versucht die Geschäftsführung bei notwendigen Erweiterung der Kapazitäten, die Zahl der Stellen unterproportional aufzustocken.

Die Mitarbeiter befürchten, dass ihr Bedürfnis nach Sicherheit nicht mehr in ausreichendem Maße befriedigt werden wird: Sie sind um die Sicherheit ihres Arbeitsplatzes und um die Höhe ihres Entgeltes ebenso besorgt wie um die Bewältigung zusätzlicher Belastungen - inhaltlicher und/oder zeitlicher Art -, von denen sie nicht wissen, ob sie diesen gewachsen sein werden. Die Mitarbeiter werden deshalb beide Arten von Veränderungen nur bedingt akzeptieren; es muss mit einer spürbaren Demotivation und als Folge davon mit Leistungseinschränkungen gerechnet werden.

Die im Krankenhaus angestellten Ärzte werden vielleicht etwas anders reagieren. Einerseits befürchten sie die Folgen der Kapazitätsänderungen wie alle anderen Mitarbeiter auch. Für Ärzte in der Weiterbildung kommt hinzu, dass diese bei dem Wegfall bestimmter Krankheitsarten ihre Weiterbildung möglicherweise nur in einem anderen Krankenhaus vervollständigen können. Wird das Leistungsspektrum des Krankenhauses dagegen um neue Krankheitsarten ergänzt, dann wird die Haltung der Ärzte eher durch die Neugierde auf die Behandlung dieser neuen Krankheitsarten geleitet.

\section{Führungskräfte}

Die Führungskräfte begrüßen einerseits die Erweiterung von Behandlungskapazitäten, wenn neue Krankheitsbilder in das Leistungsprogramm des Krankenhauses aufgenommen werden. Als Agenten ihrer Mitarbeiter werden sie aber wie diese wegen der Folgen der Kapazitätsveränderungen diese eher nur bedingt akzeptieren.

Eine der Aufgaben der Führungskräfte ist, die zu erwartende Demotivation ihrer Mitarbeiter durch geeignete Führungsleistungen zu überwinden. Die Führungskräfte sind nicht sicher, ob sie dieser Herausforderung gerecht werden können und stehen auch deshalb den Kapazitätsanpassungen eher reserviert gegenüber.

\section{Krankenkassen}

Damit ihren Versicherten ausreichende Behandlungskapazitäten mit einem befriedigenden Preis-Leistungs-Verhältnis angeboten werden können, unterstützen die Krankenkassen die erforderlichen Kapazitätsanpassungen - einerseits. Andererseits fürchten sie die durch die Erweiterung des Leistungsprogramms möglicherweise zusätzlich entstehenden Kosten; sie werden deshalb an den Nachweis der Notwendigkeit der Kapazitätsanpassungen besonders hohe Anforderungen stellen ${ }^{36}$.

\section{Eigentümer}

Der Eigentümer ist einerseits daran interessiert, der Bevölkerung im Einzugsbereich des Krankenhauses den uneingeschränkten Zugang zu medizinischen Leistungen anbieten zu können. Auf der anderen Seite erwartet er zum Zwecke der Vermeidung von Effizienzdefiziten den Abbau überflüssiger Kapazitäten. Er wird deshalb die Pläne der Geschäftsführung zwecks Anpassung der Behandlungskapazitäten an die sich ändernden Anforderungen unterstützen.

36 Siehe zum Beispiel den Brief der Arbeitsgemeinschaft der Krankenkassen und Krankenkassenverbände in Berlin an den Staatssekretär in der Senatsverwaltung für Gesundheit, Umwelt und Verbraucherschutz in Berlin vom 9. Februar 2009 zu der Fortschreibung der Krankenhausplanung 2010. 


\subsubsection{Technologischer Wandel}

Immer leistungsfähigere medizintechnische Geräte sowie informations- und kommunikationstechnische Systeme ermöglichen eine präzisere und schnellere Diagnostik und einen größeren therapeutischen Nutzen, und dies vor allem auch für immer ältere und alte Patienten. Diese Entwicklung verlangt von den Krankenhäusern eine Entscheidung darüber, ob und in welchem Umfang sie sich dieser Entwicklung durch entsprechende Investitionen anschließen und sie sogar fördern wollen. Die Alternative ist, auf die Entwicklung eigener High-Tech-Leistungsbereiche $\mathrm{zu}$ verzichten und gewisse medizin-technische Leistungen, die nur mit einem sehr hohen technischen Aufwand realisiert werden können - wie zum Beispiel die diagnostischen Leistungen bildgebender Verfahren -, von den dafür spezialisierten Krankenhäusern - mit gegebenenfalls geringeren Kosten - einzukaufen.

Die modernen Informations- und Kommunikationssysteme ermöglichen den effizienten und bequemen Austausch großer Mengen an Daten sowohl innerhalb des Krankenhauses als auch zwischen dem Krankenhaus und anderen Anbietern medizinischer Leistungen (niedergelassene Ärzte, Rehabilitationskliniken, Einrichtungen der stationären und ambulanten Pflege und andere). Deren Bedeutung wird in Zukunft noch steigen, weil angesichts der zunehmenden Spezialisierung in der Medizin die Zahl der Personen/Institutionen zunehmen wird, die an der Patientenbehandlung beteiligt sind und zwischen denen behandlungsrelevante Daten als Voraussetzung für eine sichere Behandlung auf hohem qualitativen Niveau ausgetauscht werden müssen.

Mittels der modernen Informations- und Kommunikationssysteme werden nicht nur die Patientenbehandlung innerhalb des Krankenhauses und die Weiterbehandlung durch andere Leistungsanbieter sowie die Managementprozesse nachhaltig unterstützt (Beispiel 1); Effektivität, Sicherheit und Effizienz werden kontinuierlich gesteigert (Beispiel 2). Sie sind eine Voraussetzung für die Realisierung sektorübergreifender Organisationsformen der Patientenbehandlung (wie zum Beispiel Integrierte Versorgung nach $\$ 140 a-d$ Sozialgesetzbuch (SGB) V).

Die Transparenz der Behandlungs-, Serviceund Betriebsführungsprozesse wird verbessert mit der Folge, dass Entscheidungen und/oder
Handlungen der Geschäftsführung und/oder der Instanzen, die dieser nachgeordnet sind, sehr viel leichter - vor allem auch von den Betroffenen verfolgt werden können. Die Geschäftsführung und ihre Mitarbeiter müssen damit rechnen, dass sie unter ständiger Beobachtung stehen (Suchanek; Lin-Hi, S. 5).

\section{Beispiel 1}

In einer Elektronischen Patientenakte (EPA) werden alle die Krankheits- und Behandlungsverläufe eines Patienten betreffenden Daten (Befunddaten, Diagnosen, Behandlungsverlauf, Behandlungsergebnis, zugehörige Korrespondenz) gespeichert. Die sofortige Verfügbarkeit aller relevanten Behandlungsdaten macht es möglich, im Falle einer erneuten Behandlung oder der Weiterbehandlung durch einen anderen Medizinbetrieb die Anamnesezeit zu verkürzen, die Therapieentscheidung auf eine bessere Informationsbasis zu stützen und Doppeluntersuchungen gegebenenfalls zu vermeiden.

Die Qualität aller in der EPA archivierten Behandlungen kann bei jedem erneuten Einsatz von den dann behandelnden Experten beurteilt werden.

\section{Beispiel 2}

Auf der Grundlage des $\oint 1$ Krankenhausstatistik-Verordnung (KHStatV) liefern die Krankenhäuser und Vorsorgeoder Rehabilitationseinrichtungen die durch sie verursachten Kosten, die Zahl der Krankenhauspatienten und die Art ihrer Erkrankungen, differenziert nach diversen Kriterien, den zuständigen Statistischen Landesämtern; ferner übermitteln sie eine Vielzahl von Merkmalen, anhand derer der Medizinbetrieb charakterisiert werden kann.

Damit sind die Statistischen Landesämter in der Lage, die Qualität und die Effizienz der Leistungserbringung in den Medizinbetrieben zu beurteilen - wenn auch die Ergebnisse dieser Analysen mit gewissen Vorbehalten gesehen werden müssen. Die Statistischen Landesämter können den Krankenhäusern wichtige Informationen liefern, die diese - mit gewissen Einschränkungen - für die Weiterentwicklung der Qualität und Effizienz der Patientenbehandlung verwenden.

Der Spielraum für Entscheidung der Geschäftsführung, die Medizin-Technik sowie die Informations- und Kommunikationstechnologie betreffend, wird unter anderem von folgenden Stakeholder-Anliegen bestimmt: 


\section{Stakeholder und deren Anliegen, bezogen auf den technologischen Wandel}

\section{Entscheidung der Geschäftsführung}

Die Geschäftsführung beschließt, in die Modernisierung der Medizin-Technik und in den Ausbau der vorhandenen Informations- und Kommunikationssysteme zu investieren. Dieses schließt das Schaffen der Nutzung einer Elektronischen Patientenakte (EPA) und das Einbinden der niedergelassenen Ärzte in das Intranet des Krankenhauses ein.

\section{Stakeholder und Stakeholder-Anliegen}

\section{Patienten}

Patienten haben einerseits ein großes Interesse daran, dass sie auf der Grundlage der neuesten Erkenntnisse der Medizin und unter Verwendung neuester Entwicklungen der Medizin-Technik behandelt werden. Auf der anderen Seite wird der Einsatz der Medizintechnik nicht uneingeschränkt gut geheißen. Die medizin-technischen Geräte werden eher als Barriere zwischen dem Patienten und dem Arzt sowie der Pflegekraft wahrgenommen; die Patienten vermissen unter bestimmten Voraussetzungen die mitfühlende Hand des Arztes und die helfende Hand der Pflegekraft (s. Kap. 4.2.2, Praxis). Insofern werden die Patienten nicht einheitlich mit ihrer Nachfrage nach medizinischen Leistungen eines Krankenhauses positiv auf die Erweiterung der medizin-technischen Ausstattung in diesem reagieren.

Mit der Verbesserung des Informationsaustausches innerhalb des Krankenhauses erhoffen sich die Patienten die weitere Steigerung der Sicherheit, der Qualität und der Effizienz der Behandlung sowie die Straffung der Arbeitsabläufe, sodass ihre Verweildauer im Krankenhaus ausschließlich durch die Art ihrer Erkrankung und deren Behandlung sowie unter Berücksichtigung ihrer Gesamtkonstitution, nicht aber zusätzlich durch organisatorische Aspekte bestimmt wird. Bei der Entscheidung, welches Krankenhaus die Patienten im Bedarfsfall aufsuchen werden, spielen diese Kriterien eine entscheidende Rolle.

Die Erweiterung der Kommunikationsmöglichkeiten zwischen den verschiedenen Medizinbetrieben durch die Einführung der EPA wird von den Patienten unterschiedlich beurteilt:

- Einerseits wird die Weitergabe von Behandlungsdaten von einem Medizinbetrieb zu einem anderen mittels der EPA von den Patienten begrüßt. Sie verbinden damit die Hoffnung, dass die Qualität der Behandlung und vor allem die Behandlungssicherheit für den Fall, dass sie nach der stationären Behandlung ambulant durch den Hausarzt, in einer Rehabilitationsklinik oder einem anderen Medizinbetrieb weiter behandelt werden müssen, weiter verbessert werden. Der Medizin- betrieb, der nicht auf die flächendeckende Einführung der EPA wartet, sondern diese für sein Unternehmen anbietet, verschafft sich - bei positiver Beurteilung durch die Patienten - einen Wettbewerbsvorteil und bindet Patienten an sein Unternehmen ${ }^{37}$.

- Die EPA wird aber nicht nur positiv beurteilt. Es wird befürchtet, dass die dort gesammelten Daten auch für andere Zwecke verwendet werden (können).

Insofern ist noch offen, wie die Patienten auf die Einführung der EPA reagieren werden - ob sie darin eher die Befriedigung ihres Bedürfnisses nach weiter verbesserter Behandlungsqualität sehen oder ob die erwähnten Befürchtungen die Oberhand behalten werden.

\section{Bevölkerung im Einzugsbereich des Krankenhauses}

Die Bevölkerung im Einzugsbereich des Krankenhauses wird eine Entscheidung der Geschäftsführung für die Modernisierung der technischen Ausstattung begrüßen. Sie geht davon aus, dass die Behandlungsqualität - und damit ist auch gemeint, dass eventuell zusätzliche Diagnose- und Therapieformen in der Nähe ihrer Wohnung angeboten werden - weiter zunehmen wird. Wenn das Krankenhaus in ihrer Nachbarschaft mittels telemedizinischer Systeme an ein Zentral-Krankenhaus angebunden wird und auf diese Weise zumindest die Diagnose einer Erkrankung vor Ort unterstützt werden kann, wird das als großer Zuwachs an Behandlungssicherheit und -qualität begrüßt (eventuelle Nachteile werden erst als Patient wahrgenommen).

Hinsichtlich der Weiterentwicklung des Informationsaustausches zwischen den Medizinbetrieben werden die Menschen im Einzugsbereich des Krankenhauses ähnlich reagieren wie die Patienten.

\section{Niedergelassene Ärzte}

Die niedergelassenen Ärzte vertreten zwei sehr unterschiedliche Positionen:

- Im Interesse ihrer Patienten fordern die einweisenden Ärzte die Anpassung der medizin-technischen Ausrüstung an den neusten Stand der Medizin-Technik.

Sie fordern aus guten Gründen die rasche Übermittlung aller Behandlungsdaten für die von ihnen in ein Krankenhaus eingewiesenen Patienten. Im Zusammenhang mit der Entscheidung, in welches Krankenhaus die niedergelassenen Ärzte ihre Patienten einweisen, spielt die Befriedigung dieses Bedürfnisses eine entscheidende Rolle.

37 Die RHÖN-KLINIKUM AG vernetzt ihre 46 Kliniken mit Hilfe einer webbasierten EPA (WebEPA). Damit sollen in Zukunft die konzernweit jährlich mehr als 1 Million behandelten Patienten von nachweislich höherer Versorgungsqualität profitieren (siehe die gemeinsame Presseinformation - RHÖN-KLINIKUM AG und Siemens AG Medical Solutions - vom 18. September 2007). 
- Die Einführung der EPA wird von ihnen dagegen seit Jahren blockiert (Germis, o.S.). Über die Gründe für dieses Verhalten kann man derzeit nur spekulieren. Einer der Gründe dürfte jedoch der sein, dass die Ärzte durch den Austausch aller patientenrelevanten Daten mit anderen Leistungserbringern nicht ständig unter Beobachtung stehen und nicht kontrolliert werden wollen.

\section{Mitarbeiter}

Mitarbeiter (Ärzte, Pflegekräfte, Therapeuten und Vertreter anderer Berufsgruppen) sind von der Erweiterung des Technikeinsatzes unterschiedlich betroffen:

- In ihrer Rolle als Agent der Patienten erwarten sie die Befriedigung ihres Bedürfnisses nach weiterer Verbesserung der Behandlungsqualität, vor allem wegen des einfacheren Zugriffs auf die für einen Behandlungsfall relevanten Informationen. Als Wissenschaftlicher begrüßen sie eine weitere Erleichterung des Zugangs zu forschungsrelevanten Informationen.

- Das Beherrschen komplizierter medizin-technischer Verfahren setzt besondere Fähigkeiten voraus. Die Aufgabenträger genießen deshalb ein besonders hohes Maß an Prestige. Ihr Bedürfnis nach Anerkennung, Aufmerksamkeit und Status, aber auch nach Selbstvertrauen, Können und Wissen werden in besonderem Maße befriedigt. Insofern kann die Geschäftsführung mit der Unterstützung ihrer Pläne durch eine größere Zahl von Mitarbeitern rechnen.

- Die Mitarbeiter müssen sich andererseits auf

- andere Arbeitsabläufe,

- neue Anforderungen, die sich aus dem Einsatz der Technik ergeben,

- die Bewältigung der Folgen, die aus der Weiterentwicklung der Medizin-Technik und der dadurch nicht selten ausgelösten Zunahme der Spezialisierung resultieren - wie zum Beispiel die Einschränkung des Entscheidungs- und Handlungsspielraums, die Einschränkung der sozialen Interaktion und Kommunikation sowie die Zunahme der Monotonie der auszuführenden Tätigkeiten -, sowie auf - die Bewältigung neuer Schnittstellenprobleme einstellen.

- Hinzu kommt, dass - wenn die Verfügbarkeit über eine größere Menge an Daten nicht zu Entscheidungsunsicherheiten führen soll - die mit Entscheidungsbefugnis ausgestatteten Mitarbeiter festlegen müssen, mit welchen entscheidungsrelevanten Daten sie versorgt werden wollen; erfahrungsgemäß sind dazu nicht alle Mitarbeiter im Stande.

Die Bedürfnisse der Mitarbeiter nach Sicherheit (= nach Bewältigenkönnen der sich möglicherweise ändernden Anforderungen), nach Zugehörigkeit (= nach gefühlsbetonten Kontakten mit anderen Per- sonen und nach einem akzeptierten Platz innerhalb einer Gruppe) (Scholz, S. 878) werden möglicherweise nicht in dem Maße befriedigt, wie es die Mitarbeiter vor der Erweiterung des Technikeinsatzes gewohnt waren. Sie befürchten, ständig beobachtet und kontrolliert zu werden.

- Wenn das Krankenhaus die Rolle als „Technologie-Führer" anstrebt, sind davon Mitarbeiter - vor allem Ärzte - mit weiteren, bisher noch nicht erwähnten und unterschiedlichen Bedürfnissen betroffen. Die einen engagieren sich vor allem für die Entwicklung und Implementierung neuer medizin-technischer Geräte und der darauf aufbauenden Behandlungsmethoden. Für die andere Gruppe von Mitarbeitern steht die situativ, stark emotional geprägte Interaktion zwischen den Mitgliedern des therapeutischen Teams und den Patienten im Mittelpunkt ihres Handelns (Naegler et al. 2008, S. 7); die medizin-technischen Geräte werden dabei eher als Barriere zwischen dem Patienten und dem Arzt wahrgenommen.

Die Geschäftsführung muss deshalb damit rechnen, dass Veränderungen an der medizin- und/oder informationsund kommunikationstechnischen Ausstattung nicht von allen Mitarbeitern akzeptiert werden.

\section{Führungskräfte}

Der technologische Wandel hat bereits dazu geführt und wird weiter dazu beitragen, dass zusätzlich zu den traditionell im Krankenhaus beschäftigten Berufsgruppen neue Berufe kreiert werden (unter anderem Casemanager und Dokumentationsassistenten). Er führt zu einer weiteren Spezialisierung der verschiedenen Leistungsbereiche und der darin arbeitenden Mitarbeiter. Das Zusammenwirken hoch spezialisierter Know-how-Träger verlangt von den Führungskräften die Koordination einer spezifischen Art von Teamarbeit bei zunehmender Kommunikationsintensität (von Eiff 2000, S. 52). Der Ausgleich der Spannungen, die durch das Zusammenwirken sehr unterschiedlicher Persönlichkeitsstrukturen entstehen können, stellt die Führungskräfte vor besondere Herausforderungen und verlangt den Einsatz geeigneter Führungsinstrumente.

Die Führungskräfte sind nicht sicher, ob sie den daraus resultierenden geänderten Anforderungen gerecht werden können. Es wird Führungskräfte geben, die befürchten wie ihre Mitarbeiter -, dass ihr Wunsch nach Sicherheit (= nach Bewältigenkönnen der sich möglicherweise ändernden Anforderungen) nur bedingt erfüllt wird. Hinzu kommen die Vorbehalte, die auch ihre Mitarbeiter im Zusammenhang mit einer weiteren Technisierung des Krankenhauses artikuliert haben.

Es muss deshalb einerseits mit einer gewissen Reserviertheit auch von Führungskräften gerechnet werden. Sie werden andererseits die Modernisierung der technischen 
Ausstattung des Krankenhauses als Basis einer weiter steigenden Behandlungsqualität begrüßen; sie werden sich auch die weitere Effektuierung ihrer wissenschaftlichen Arbeit versprechen.

Die Führungskräfte sind dem Unternehmen verpflichtet, die Mitarbeiter im Sinne der Unternehmensziele zu motovieren; sie sind allerdings nicht sicher, ob sie diese Aufgabe mit Erfolg wahrnehmen können. Andererseits sind sie solidarisch mit ihren Mitarbeitern und werden diese möglicherweise in der oben skizzierten Haltung unterstützen. Es ist also noch offen, ob die Geschäftsführung unter diesen Aspekten mit der Unterstützung aller Führungskräfte rechnen kann.

\section{Krankenkassen}

Der Fortschritt der Medizin und der der Medizin-Technik führt zu einer steigenden Nachfrage nach einschlägigen Diagnose- und Therapieleistungen sowie zu steigenden Kosten je Diagnose- und Therapieeinheit und damit zu steigenden Betriebs- und Investitionskosten (Schmidt; Möller 2007, S. 7). Die Krankenkassen und damit die Solidargemeinschaft werden versuchen, die zusätzlichen Kosten nicht zur Gänze akzeptieren zu müssen. Das künftig medizinisch und medizin-technisch Mögliche wird den Patienten unter Umständen nicht in vollem Umfang zur Verfügung gestellt werden können. Dieser Versuch wird von den Krankenkassen auch deshalb unternommen, weil nicht immer nachgewiesen wird (werden kann), ob das medizinisch und medizin-technisch zusätzlich Mögliche einen die zusätzlichen Kosten rechtfertigenden Zusatznutzen für die Patienten zur Folge hat.

Die Krankenkassen werden deshalb die Pläne der Geschäftsführung eher mit Skepsis begleiten.

Andererseits haben die Krankenkassen aus Gründen des Wettbewerbs zwischen den Krankenkassen ein großes Interesse daran, dass ihren Versicherten die diagnostischen und therapeutischen Leistungen nach dem jeweils neuesten Stand medizinischer und medizin-technischer Erkenntnisse zugutekommen.

Nur mit Hilfe der Weiterentwicklung der Informations- und Kommunikationstechnologie und deren Einsatz kann die große Menge an Daten bewältigt werden, die auf der Grundlage des $\varnothing 21$ Krankenhausentgeltgesetzes (KHEntgG) an eine zentrale Stelle geliefert werden müssen. Anhand dieser auf das Krankenhaus bezogenen Strukturdaten sowie sämtlicher Falldaten können detaillierte Leistungs- und Kosten-Vergleiche durchgeführt und die Leistungs- und Kosten-Entwicklung in jedem Krankenhaus beobachtet werden. Die Krankenkassen begrüßen deshalb die Weiterentwicklung der Informations- und Kommunikationstechnologie und deren Einsatz.

\section{Eigentümer}

Der Eigentümer unterstützt die Modernisierung der technischen Ausstattung, weil dieses zu einer weiteren Verbesserung der Behandlungsqualität und damit zu einer weiteren Verbesserung der Reputation des Krankenhauses und damit schließlich zu einer Zunahme der Inanspruchnahme und zu steigenden Erlösen führt. Der Eigentümer unterstützt die Weiterentwicklung der Informations- und Kommunikationstechnologie und deren Einsatz auch deshalb, weil er sich auf deren Grundlage einen umfassenderen Einblick in das betriebliche Geschehen verspricht.

Der zu erwartende Anstieg der Betriebs- und Investitionskosten und die auch zu erwartende Weigerung der Krankenkassen, diese in vollem Umfang in die Fallpauschalen zu übernehmen, schmälern den Spielraum für die Verzinsung des von dem Eigentümer zur Verfügung gestellten Kapitals. Der Eigentümer wird deshalb seinerseits versuchen, den Anstieg der Kosten zu bremsen, indem den Patienten zwar die Leistungen, die zur erfolgreichen Behandlung notwendig sind, zur Verfügung gestellt werden, nicht aber die Leistungen, die darüber noch möglich wären.

\subsubsection{Organisatorischer Wandel}

\section{Formen organisatorischen Wandelns im Überblick}

Die Behandlungs-, Support- und Betriebsführungsprozesse bedürfen der Organisation, damit die mit Hilfe der genannten Prozesse beabsichtigten Ziele erreicht werden können. Dabei wird unter Organisation einerseits die ordnende Tätigkeit und zum anderen das Ergebnis dieses Handelns, nämlich die Struktur und die Arbeitsabläufe (= Prozesse) verstanden.

Ausgangspunkt des Organisierens ist die $\mathrm{Ge}$ samtaufgabe des Unternehmens. Dabei kann als Unternehmen sowohl - auf der Mesoebene - ein Medizinbetrieb verstanden werden, in dem die stationäre sowie die ambulante Behandlung und andere Leistungen angeboten werden, als auch auf der Makroebene - das Gesundheitssystem als Ganzes, in dem die Krankenbehandlung von mehreren Leistungsanbietern realisiert wird. Die folgenden Ausführungen beziehen sich auf beide Arten von Unternehmen.

Das Ergebnis des Organisierens ist abhängig von der zu bewältigenden Aufgabe und von diversen internen Bedingungen, unter denen die Leistungserbringung erfolgt (dazu mehr in Kap. 4.2) sowie von diversen extern gesetzten Rahmenbedingungen (dazu mehr in Kap. 4.4). Insofern erfolgt die Veränderung der Unternehmensstruktur 
einerseits proaktiv, indem aktuelle Erkenntnisse und Erfahrungen aus Wissenschaft und Praxis umgesetzt werden. Zum anderen reagiert die Geschäftsführung auf Veränderungen der extern gesetzten Rahmenbedingungen.

Verbesserungen struktureller und/oder prozessualer Art sind Schlüsselfaktoren zur weiteren Steigerung der Ergebnisqualität sowie der Effizienz der Behandlungsprozesse und können im Regelfall nicht substituiert werden (wie dies z.B. im Zusammenhang mit der Weiterentwicklung der Medizin-Technik beschrieben worden ist). Sie tragen zur Stärkung der Wettbewerbsfähigkeit und damit zur langfristigen finanziellen Sicherung des Unternehmens bei.

Krankenhausinterne und Leistungsanbieter übergreifende organisatorische Veränderungen sind aktuell in mehrfacher Hinsicht zu beobachten:

1. Innerhalb des Krankenhauses findet eine allmähliche Umorientierung von der funktional orientierten zu einer mehr an den Behandlungsprozessen ausgerichteten Organisation statt. Krankenhäuser sind durch zunehmende Spezialisierung geprägt - Spezialisierung sowohl im Sinne einer weiteren Ausdifferenzierung großer Fachgebiete in Teilgebiete (So gibt es zum Beispiel vor allem in Krankenhäusern der Schwerpunkt- und Maximalversorgung nicht mehr nur eine Abteilung „Innere Medizin“, sondern mehrere mit jeweils einem Schwerpunkt wie z.B. Gastroenterologie, Kardiologie oder Nephrologie.) als auch im Sinne der Konzentration auf bestimmte Fach- bzw. Teilgebiete und Verzicht auf das Angebot anderer Fach- bzw. Teilgebiete. Diese objektorientierte Spezialisierung ist nicht zuletzt dem immer lauter werden Ruf nach Mindestmengen an Behandlungen einer bestimmten Fallgruppe geschuldet.

2. Die strikte Trennung der Patientenversorgung in einerseits stationäre und andererseits ambulante Versorgung wird allmählich überwunden. Die Grenzen zwischen den Sektoren des Gesundheitssystems werden durchlässiger.

3. Vor allem der Druck, der von veränderten finanziellen Rahmenbedingungen ausgeht, führt dazu, dass Krankenhäuser untereinander unterschiedliche Koalitionen eingehen.

4. Die neue Arbeitszeitordnung für Ärzte hat beträchtliche Auswirkungen auf die Strukturen des Krankenhauses und die Arbeitsabläufe in diesem.
Aus Platzgründen können von den sich abzeichnenden Veränderungen nur die unter der Ziffer 1. genannten in die Betrachtungen der folgenden Abschnitte aufgenommen werden.

\section{Krankenhausinterne Veränderungen}

Die Patientenbehandlung findet im Regelfall immer noch in Leistungsbereichen statt, die in personeller, finanzieller, struktureller und organisatorischer Hinsicht von den jeweils anderen Leistungsbereichen eines Krankenhauses eindeutig abgegrenzt, also Ergebnis eines funktional orientierten Organisierens sind. Für jeden Leistungsbereich ist jeweils eine Person verantwortlich. Der Patient wird innerhalb eines derart organisierten Systems durch die an der Behandlung beteiligten Leistungsbereiche nicht selten nur als eine Summe verschiedener, zu erledigender Aufgaben wahrgenommen. Das Behandlungsziel ist in jenen Leistungsbereichen, von denen der behandelnde Leistungsbereich medizinische und nicht-medizinische Serviceleistungen erhält, nicht selten nur vage bekannt.

Krankenhausinterne Kunden-Lieferanten-Beziehungen, in deren Rahmen der Kunde - zum Beispiel die behandelnde Fachabteilung - die von einem Lieferanten - z.B. von der Röntgenabteilung - gewünschte Leistung inhaltlich und zeitlich eindeutig und unter Angabe von Qualitätskriterien beauftragt und abnimmt, gibt es nicht immer. Die Beantwortung der Frage, auf welche Weise die Bedürfnisse der internen Kunden am besten befriedigt werden können, spielt für die internen Lieferanten nicht immer in dem gewünschten Ausmaß eine Rolle (s. Beispiele). Die Folge davon ist, dass zwar innerhalb jeweils einzelner Leistungsbereiche in der Regel optimale, für den gesamten Behandlungsprozess dagegen häufig nur sub-optimale Ergebnisse, vor allem in zeitlicher Hinsicht, realisiert werden.

\section{Beispiel}

So verlangt zum Beispiel das Zentrallaboratorium die Blutproben zu einer bestimmten Zeit, ohne danach zu fragen, ob sich die Blutabnahme zu dieser Zeit in einen effizienten Arbeitsablauf der Station einfügen lässt.

Nicht selten wird das Abendessen - mit dem in der Regel nicht zutreffenden Argument, die Herstellung des Essens sonst nicht effizient gestalten zu können - am frühen 
Nachmittag produziert und den Patienten - ohne Rücksicht auf deren außerhalb des Krankenhauses gepflegten Essensgewohnheiten - zwischen 17.00 und 18.00 Uhr serviert mit der Folge, dass die Zufriedenheit der Patienten gering ist.

Die geänderten finanziellen Rahmenbedingungen (Näheres dazu s. Kap. 4.4.2, Finanzielle Rahmenbedingungen) werden - weil künftig Endprodukte abgerechnet werden - zu einer stärkeren Orientierung der Krankenhausorganisation an der Befriedigung von Patientenbedürfnissen beitragen; die Arbeitsabläufe werden mehr fallbezogen ausgerichtet sein als bisher. Einzelne Krankenhäuser überführen deshalb die funktional und an Zuständigkeiten orientierte in eine Organisation, die an dem Behandlungsprozess ausgerichtet ist. Es werden auf das Behandlungsergebnis abgestimmte, standardisierte und evidence-basierte sowie Berufsgruppen, Hierarchieebenen und Leistungsbereiche (gegebenenfalls die Grenzen des Krankenhauses) übergreifende Abläufe geschaffen und implementiert. Ein Prozesseigner - im Regelfall als Casemanager bezeichnet ist verantwortlich für die sach- und zeitgerechte Realisierung der Behandlung jeweils eines Patienten (Hildebrand, S. 49).

Der Spielraum für Entscheidungen der $\mathrm{Ge}$ schäftsführung, die den Ersatz der funktionaldurch die prozessorientierte Organisation sowie die Förderung der objektorientierten Spezialisierung zum Gegenstand haben, wird durch die im Folgenden genannten Stakeholder-Anliegen und dem Bemühen der Verantwortungsträger bestimmt, diese angemessen bei Entscheidungen zu berücksichtigen.

\section{Stakeholder und deren Anliegen, bezogen auf krankenhausinterne organisatorische Veränderungen}

\section{Entscheidung der Geschäftsführung}

Die Geschäftsführung beschließt, die funktional ausgerichtete durch eine an den Behandlungsprozessen orientierte Organisation zu ersetzen. Sofern eine weitere Spezialisierung medizinischer Fachgebiete - wie zum Beispiel die der Orthopädie oder der Frauenheilkunde - erfolgt, wird die Organisation des Krankenhauses dieser Entwicklung angepasst.

\section{Stakeholder und Stakeholder-Anliegen}

\section{Patienten, Bevölkerung im Einzugsbereich des Krankenhauses, niedergelassene Ärzte} Die an dem Behandlungsprozess orientierte Organisation des Krankenhauses führt zu der Beschleunigung der Behandlung und zu einem verbesserten Informationsaustausch zwischen den an der Behandlung beteiligten Leistungsbereichen. Beides hebt die Behandlungsqualität und -effizienz. Der Patient, der ihn einweisende Arzt sowie die Menschen, die im Einzugsbereich des Krankenhauses leben, werden diese Veränderungen begrüßen einerseits.

Von den Kritikern einer prozessorientierten Krankenhausorganisation wird andererseits geltend gemacht, dass es die klinische Abteilung und den dieser vorstehenden Leitenden Arzt, mit der sich der Patient - wie bisher - identifizieren und in deren Obhut er sich geborgen fühlen kann, nicht mehr geben wird. Zwei zentrale, den Behandlungs prozess positiv beeinflussende Elemente würden künftig fehlen. Der Patient, der ihn einweisende Arzt sowie die Menschen, die im Einzugsbereich des Krankenhauses leben, würden deshalb - so wird befürchtet - im Bedarfsfall möglicherweise ein Krankenhaus mit konventioneller Organisation bevorzugen.

\section{Mitarbeiter}

Der Ersatz der funktional durch eine an den Behandlungsprozessen ausgerichteten Organisation löst bei vielen Mitarbeitern Skepsis, Angst und Verunsicherung aus:

- Diese befürchten, dass es die ursprünglich auf größere Dauer angelegte Organisationseinheit, in der sie sich geborgen fühlen konnten, möglicherweise nicht mehr geben wird, dass ihr Bedürfnis nach Zugehörigkeit (= nach gefühlsbetonten Kontakten mit anderen Personen und nach einem akzeptierten Platz innerhalb einer Gruppe) nicht mehr befriedigt werden kann.

- Sie sind nicht sicher, ob sie den neuen Anforderungen gerecht werden können. Es muss deshalb damit gerechnet werden, dass Mitarbeiter, weil ihr Bedürfnis nach Sicherheit im Sinne des Bewältigenkönnens neuer Anforderungen nicht mehr befriedigt wird, versuchen werden, organisatorische Änderungen, wenn sie geplant werden, zu unterlaufen.

Von anderen Mitarbeitern kann erwartet werden, dass sie die Veränderungen nicht als Bedrohung wahrnehmen, sondern sich mit den Veränderungen positiv auseinandersetzen. Sie sehen die organisatorischen Veränderungen als Chance, die sie wahrnehmen und mitgestalten wollen. Diese Mitarbeiter werden einschlägige Entscheidungen der Geschäftsführung begrüßen und deren Umsetzung tatkräftig unterstützen, weil sie mit der Befriedigung ihres Selbstverwirklichungsbedürfnisses, ihres Bedürfnisses nach Ent- 
faltung der eigenen Persönlichkeit (Scholz, S. 878) rechnen; weil sie aber auch in ihrer Rolle als Agent ihrer Patienten von einer weiteren Verbesserung der Patienten-Behandlung ausgehen.

Auf eine weitere Spezialisierung werden die Mitarbeiter unterschiedlich reagieren: Der mit der objektorientierten Spezialisierung einhergehende Kompetenzzuwachs macht sie sicherer in der Wahrnehmung der ihnen übertragenen Aufgaben - insofern wirkt Spezialisierung motivationsfördernd. Spezialisierung führt andererseits zur Einschränkung der sozialen Interaktion und Kommunikation sowie zur Eintönigkeit der Aufgabendurchführung und damit eher demotivierend. Je nach ihrer Persönlichkeit werden die Mitarbeiter die Pläne der Geschäftsführung mit dem Ziel einer weiteren Spezialisierung entweder unterstützen oder zu verhindern suchen.

\section{Führungskräfte}

Die Umstellung auf die prozessorientierte Organisation des Krankenhauses wird dazu führen, dass einzelne Akteure und Funktionen an Einfluss verlieren und dass gewohnte Rollen sich verändern. Dieses betrifft vor allem die Führungskräfte. Diese sehen ihr Bedürfnis nach Wertschätzung nicht mehr befriedigt; sie befürchten zudem, dass sie an Einfluss sowohl auf die Behandlung der Patienten mit einer Erkrankung aus ihrem Fachgebiet - vor allem in organisatorischer Hinsicht - als auch auf die Gestaltung des Leistungsbereichs, in dem sie ihre Privatpatienten behandeln, verlieren. Es muss deshalb damit gerechnet werden, dass gerade von dieser Gruppe der Mitarbeiter versucht wird, die skizzierten Veränderungen zu verhindern.

Führungskräfte sind auch Agenten ihrer Patienten und in dieser Rolle an einer weiteren Verbesserung der Patienten-Behandlung interessiert. Dieses Anliegen könnte Führungskräfte dazu bewegen, die Pläne der Geschäftsführung zu unterstützen.

Die Führungskräfte sind gefordert, mittels einschlägiger Führungsleistungen dazu beizutragen, dass die in Folge organisatorischer Veränderungen bei ihren Mitarbeitern möglicherweise entstehenden Leistungs- und Motivationsdefizite vermieden werden. Sie sind allerdings nicht sicher, ob sie dieser Herausforderung gerecht werden können; ihr Bedürfnis nach Sicherheit im Sinne des Bewältigenkönnen dieser neuen Anforderungen wird nicht mehr in dem bisher gewohnten Maß befriedigt. Die Geschäftsführung kann deshalb auch deswegen nicht sicher sein, ob sie bei der Realisierung ihrer Pläne mit der Unterstützung der Führungskräfte rechnen kann.

\section{Krankenkassen; Eigentümer}

Die schon mehrfach erwähnten zu erwartenden positiven Effekte einer Umstellung auf eine prozessorientierte Krankenhausorganisation werden dazu beitragen, dass Kran- kenkassen und der Eigentümer des Krankenhauses das Vorhaben der Geschäftsführung unterstützen.

Hinsichtlich einer weitergehenden Spezialisierung und in der Folge der Konzentration einzelner Krankenhäuser auf die Behandlung ausgewählter Krankheitsbilder haben sowohl die Krankenkassen als auch die Krankenhaus-Eigentümer die Schwierigkeit, die Balance zu finden zwischen der Verbesserung der Effizienz und Qualität der Krankenbehandlung auf der einen Seite und der Erreichbarkeit eines Krankenhauses durch ihre Versicherten bzw. die Einwohner einer bestimmten Region zum anderen. Je nachdem, mit welchem Gewicht diese Entscheider ihre eigenen Bedürfnisse oder die potenzieller Patienten bei ihren unternehmerischen Entscheidungen berücksichtigen, werden sie die Absichten der Geschäftsführung in die eine oder andere Richtung unterstützen.

\section{Leistungsanbieter übergreifende Veränderungen}

Krankenhäuser, aber auch andere Leistungsanbieter suchen Partner, mit denen sie zusammen Leistungen über die gesamte Versorgungskette hinweg - nämlich von der Vorsorge über die ambulante und die stationäre Behandlung bis hin zur Rehabilitation, gegebenenfalls auch unter Einschluss der stationären und der ambulanten Pflege - anbieten können (= Versorgungsverbund) (s. Beispiel 1).

\section{Beispiel 1}

So arbeiten zum Beispiel die Charité - Universitätsmedizin Berlin und die Vitanas GmbH \& Co. KGaA im Bereich der Rehabilitation zusammen.

Mit der Realisierung von Versorgungsverbünden im Sinne von Integrierter Versorgung werden zwei Ziele verfolgt (Schellschmidt; Klauber, Robra, S. 2): 1. Einerseits sollen die Schnittstellen zwischen den verschiedenen Leistungsanbietern/Versorgungssektoren abgebaut werden, um es dem Patienten zu erleichtern, die für ihn angemessene Versorgung möglichst selbst - gegebenenfalls mit Unterstützung einer neu geschaffenen koordinierenden Stelle, dem Casemanager - organisieren zu können. Die bisher existenten Schnittstellen werden ersetzt durch Schnittstellen zwischen jeweils einem Leistungsanbieter und der die Zusammenarbeit zwischen den Leistungsanbietern koordinierenden Stelle. 
2. Zum anderen will man dadurch eine hohe Versorgungsqualität und ein günstiges PreisLeistungs-Verhältnis gewährleisten.

Krankenhäuser begründen mit anderen Krankenhäusern strategische Allianzen, indem sie Leistungsbereiche des medizinischen und nicht-medizinischen Supports zusammenlegen. Auf diese Weise erhofft man sich eine Steigerung der Qualität und der Effizienz der Leistungserbringung (s. Beispiel 2).

\section{Beispiel 2}

Die Charité - Universitätsmedizin Berlin und Vivantes Netzwerk für Gesundheit $\mathrm{GmbH}$ werden ihre jetzt noch in jedem Unternehmen existierenden Leistungsbereiche „Laboratoriumsmedizin" in einer dafür gegründeten Gesellschaft zusammenführen.

Krankenhäuser gründen zusammen mit Fachfirmen verschiedener Dienstleistungsbereiche (wie zum Beispiel Reinigung, Catering und/oder Facility Management) Tochterfirmen und kaufen von diesen Tochterfirmen Dienstleistungen, die sie vorher in eigenen Leistungsbereichen $\mathrm{zu}$ im Regelfall weniger günstigen Konditionen selbst erbracht haben (s. Beispiel 3).

\section{Beispiel 3}

Siehe zum Beispiel die Charité CFM Facility Management $\mathrm{GmbH}$, die 51-prozentige Tochter der Charité - Universitätsmedizin Berlin, die ab dem 1. Januar 2006 den überwiegenden Teil der nicht-medizinischen Dienstleistungen für die Charité erbringt.

Von Entscheidungen dieser Art - im Folgenden wird aus Platzgründen nur die erste Kategorie von Leistungsanbieter übergreifenden Veränderungen berücksichtigt - sind diverse Personen und Institutionen wie folgt betroffen.

\section{Stakeholder und deren Anliegen, bezogen auf Leistungsanbieter übergreifende Veränderungen}

\section{Entscheidung der Geschäftsführung}

Die Geschäftsführung beschließt, zusammen mit den Fachärzten für die Fachgebiete X, Y, Z ein Medizinisches Versorgungszentrum (MVZ) mit Sitz am Krankenhaus zu gründen.

\section{Stakeholder und Stakeholder-Anliegen}

\section{Patienten, Bevölkerung im Einzugsbereich des Krankenhauses}

Die Patienten und die Bewohner im Einzugsbereich eines Versorgungsverbundes erwarten - vor allem dann, wenn sie wegen der Art ihrer Erkrankung verschiedene Leistungsanbieter in Anspruch nehmen müssen - eine Verbesserung der Versorgung auf unterschiedlichen Ebenen (Weatherly et al., S. 15f.):

- Sie sind überzeugt davon, dass eine höhere Ergebnisqualität erzielt werden kann;

- sie erwarten eine höheres Maß an Sicherheit als Ergebnis besser koordinierter Prozesse und

- sie schätzen die Bequemlichkeit der Behandlung auf der Grundlage abgestimmter und optimierter Prozesse; im Bedarfsfall werden sie ohne Umwege an die für die Behandlung ihrer Erkrankung adäquate Instanz verwiesen.

Die Bewohner im Einzugsbereich eines Versorgungsverbundes verbinden mit dessen Einrichtung die Hoffnung, dass dieser helfen könnte, den sich - demografisch bedingt - abzeichnenden Mangel an Ärzten für die ambulante Versorgung zu überwinden. Der Versorgungsverbund stellt attraktive Arbeitsplätze zur Verfügung, die mit den Lebensentwürfen des ärztlichen Nachwuchses eher zur Deckung zu bringen sind als die tradierten, vor allem im ländlichen $\mathrm{Be}$ reich noch vorherrschenden Strukturen für die ambulante Versorgung (Schäg et al., S. 81). Sie werden auch deshalb die Einrichtung einer Integrierten Versorgung begrüßen.

In welchem Ausmaß allerdings die (potenziellen) $\mathrm{Pa}$ tienten das Angebot der Integrierten Versorgung tatsächlich nutzen werden, ist noch offen. Immerhin geben sie mit dem Einschreiben in einen Versorgungsverbund als Voraussetzung für das Nutzen der genannten Vorteile ihre Freiheit auf, im Bedarfsfall den behandelnden Arzt und/ oder das Krankenhaus wählen zu können.

Welches der skizzierten Bedürfnisse von den (potenziellen) Patienten künftig wie bewertet und die Entscheidung für den Beitritt zu einem Versorgungsverbund leiten wird, bleibt abzuwarten.

\section{Niedergelassene Ärzte}

Sofern der Versorgungsverbund als ein Modell der Integrierten Versorgung gestaltet wird, ist der einweisende Arzt Teil des Systems und an seiner Entstehung maßgeblich beteiligt gewesen. Er ist in die Behandlung "seiner" Patienten - unabhängig davon, durch welchen Partner des Versorgungsverbundes diese jeweils erfolgt - immer eng eingebunden. Er verspricht sich eine Verbesserung der Versorgung seiner Patienten sowie die Vergütung seiner Leistungen außerhalb des Budgets. Sein Bedürfnis nach Sicherheit, nämlich nach Sicherheit, dass "seine“ Patienten 
bestmöglich versorgt werden, und nach einem akzeptierten Platz für sich innerhalb einer Gruppe werden befriedigt. Weil in einem Versorgungsverbund im Regelfall eine größere Zahl von Ärzten zusammenarbeitet, lassen sich Vertretungen leichter organisieren; der Arzt kann seiner Verantwortung gegenüber seiner Familie besser nachkommen. Schließlich kann er die Gewissheit haben, dass die von ihm aufgebaute Praxis, sein Lebenswerk, in seinem Sinne weitergeführt wird.

Es gibt also viele Gründe, die für den niedergelassenen Arzt für die Einrichtung eines Versorgungsverbundes sprechen.

Nicht alle Ärzte werden bereit sein, Mitglied eines Versorgungsverbundes zu werden, weil sie damit ein hohes Maß an Eigenständigkeit aufgeben.

\section{Mitarbeiter}

Die mit der Gründung eines Versorgungsverbundes erwartete Effizienzsteigerung kann nur realisiert werden, wenn die Versorgungsleistungen mit einem gegenüber der konventionellen Organisation der Versorgung geringeren Ressourceneinsatz erbracht werden. Die Basis dafür sind Behandlungsleitlinien, die die gesamte Behandlungskette umfassen, und darauf aufbauende Optimierungen der Behandlungs- und Supportprozesse. Einzelne Verbundpartner werden sich als Voraussetzung für die Steigerung der Behandlungs-Effizienz von einigen ihrer Mitarbeiter trennen (müssen).

Die Mitarbeiter der verschiedenen Leistungsbereiche müssen sich auf neue Aufgabenstellungen und neue Arbeitsabläufe und damit neue Anforderungen an ihre Fähigkeiten einstellen.

Der Wunsch der Mitarbeiter nach Sicherheit, also erstens nach Erhalt des Arbeitsplatzes mit einer auskömmlichen Vergütung und zweitens nach Bewältigenkönnen neuer Anforderungen, wird möglicherweise nicht mehr erfüllt. Auf der anderen Seite erwarten die Mitarbeiter, dass eines ihrer Individualbedürfnisse, nämlich die Wahrnehmung ihrer Rolle als Agent der Patienten und die dieser Rolle geschuldeten bestmöglichen Versorgung der Patienten, besser befriedigt wird.

Mitarbeiter werden - weil sie der Meinung sind, dass die erwarteten Nachteile gegenüber dem Vorteil, mit dem sie rechnen können, deutlich überwiegen - der Gründung eines Versorgungsverbundes eher skeptisch entgegen sehen. Sie werden versuchen, die Veränderungen zu verhindern.

Es wird andererseits Mitarbeiter geben, von denen erwartet werden kann, dass sie die Veränderungen nicht als Bedrohung wahrnehmen, sondern sich mit den Veränderungen positiv auseinandersetzen. Sie sehen die organisatorischen Veränderungen als Chance, die sie wahrnehmen und mitgestalten wollen, weil sie mit der Befriedigung ihres Selbstverwirklichungsbedürfnisses, ihres Bedürfnisses nach Entfaltung der eigenen Persönlichkeit (Scholz, S. 878) rechnen. Sie werden die Geschäftsführung eher ermuntern, die für die Gesellschaft insgesamt vorteilhaften Entwicklungen einzuleiten und umzusetzen.

\section{Führungskräfte}

Die Führungskräfte sehen die bisher schon skizzierten Vorteile eines Versorgungsverbundes. Sie werden deshalb die Geschäftsführung bei der Realisierung ihrer diesbezüglichen Pläne unterstützen.

Andererseits sind sie nicht sicher, ob sie die zum Erhalt der Motivation ihrer Mitarbeiter erforderlichen Führungsleistungen erbringen können. Sie befürchten, dass ihr Bedürfnis nach Sicherheit im Sinne des Bewältigenkönnen neuer Anforderungen künftig weniger gut befriedigt wird. Sie werden nach Abwägen der Vor- und der Nachteile der beabsichtigten Änderungen diesen eher skeptisch gegenüberstehen.

\section{Krankenkassen}

Die Krankenkassen lassen sich als Vertragspartner für den Aufbau der neuen Versorgungsform aus mehreren Gründen gewinnen (Weatherly et al., S. 19ff.):

- Sie versprechen sich durch ihre Beteiligung einen Wettbewerbsvorteil.

- Sie wollen dazu beitragen, die Versorgung ihrer Versicherten zu verbessern

- Sie wollen Know-how im Sinne von Versorgungskompetenz gewinnen, um eine gestaltende Rolle und nicht nur die Rolle als Finanzier spielen zu können.

- Die Kosten für die Versorgung sollen reduziert werden.

\section{Eigentümer}

Der Eigentümer des Krankenhauses, dessen Management die Gründung eines Versorgungsverbundes im Sinne der Integrierten Versorgung beschließt, wird diese Entscheidung mit tragen, weil

- die Versorgung der Patienten verbessert werden kann,

- die dadurch gesteigerte Reputation des Krankenhauses dauerhaft zu mehr Inanspruchnahme mit höheren Erlösen führt,

- die Auslastung des Krankenhauses und damit die Effizienz der Versorgung verbessert werden können und somit

- am ehesten dazu beigetragen werden kann, die sich abzeichnenden Probleme der regionalen Krankenversorgung, die auch in der Bedrohung einzelner Krankenhäuser bestehen können, zu überwinden (Neubauer et al., S. $65 f f$.$) ,$ 


\subsubsection{Wertewandel}

Werte sind kognitive Präferenzstrukturen, die Orientierung geben und als Entscheidungsregeln das Verhalten steuern (Scholz, S. 18f.). Werte sind Aspekte der Lebens- und Handlungswirklichkeit, denen für das menschliche Leben, auch im Sinne des Arbeitslebens, eine Bedeutung zugeschrieben wird und die deshalb als erstrebens- und schützenswert gleichermaßen angesehen werden (Steinkamp; Gordijn, S. 49). Das in einem Krankenhaus gelebte Wertesystem steuert sowohl den Umgang der Mitarbeiter untereinander als auch das Verhalten der Institution Krankenhaus und der Mitarbeiter gegenüber Patienten, Angehörigen, einweisenden Ärzten, Lieferanten und anderen Stakeholdern und umgekehrt das Verhalten der zuletzt genannten Stakeholder gegenüber den Mitarbeitern sowie deren Führungskräften.

Zu den Werten zählen unter anderem

- das Lebens,

- die Gesundheit,

- die Menschenwürde,

- die Freiheit,

- die Gerechtigkeit,

- die Solidarität,

- das Recht auf Arbeit sowie

- das Ende der Verschwendung (Spiegel, S. 11ff.)

Neben diesen Werten, die mehr allgemeingültigen Charakter haben, gibt es zwei Kategorien von Werten, die im Rahmen der vorliegenden Untersuchung von besonderem Interesse sind:

- Die Autonomie der Patienten im Behandlungsprozess (Bobbert, S. 76ff.) wird in Kapitel 4.2.4 zum Thema gemacht.

- Als Grundlage für die Gestaltung der Arbeitsbedingungen gibt es ein spezifisches Wertesystem, das unter anderem aus folgenden Elementen besteht (Scholz, S. 21; Spiegel, S. 111ff. und 133ff.) und das bei den weiteren Ausführungen vordringlich interessiert.

- Selbstentfaltung,

- Demokratiebewusstsein,

- Individualisierung,

- Karriereorientierung und

- Selbstständigkeit.

Charakteristisch für die genannten Werte ist es, dass deren Bedeutung abhängig ist von der Gesellschaftsordnung, in der sie gelebt werden, und dass deren Bedeutung sich im Zeitablauf ändern kann. Letzteres ist seit geraumer Zeit für mehrere Werte zu beobachten. So ist zum Beispiel die Patientenautonomie eine Größe geworden, die aus den Krankenhäusern nicht mehr wegzudenken ist (Steinkamp; Gordijn, S. 84). An der Befriedigung des Bedürfnisses der Mitarbeiter nach mehr Selbstentfaltung, mehr Demokratie am Arbeitsplatz und Selbstständigkeit kommen die Geschäftsführungen nicht mehr vorbei. Es ist anzunehmen, dass diese Elemente des Wertesystems noch längere Zeit Gültigkeit haben werden und dass sie somit Anlass sein sollten für nachhaltig wirkende strukturelle und prozessuale Anpassungen.

Konkrete Handlungen schreiben Werte nicht vor. Damit sie dennoch ihre Wirkung dauerhaft entfalten können, werden aus ihnen Handlungsanweisungen (= Normen) abgeleitet, die den Werten so gut als möglich Rechnung tragen. Entsprechend der oben für dieses Kapitel vorgenommenen Einschränkung des Untersuchungsgegenstandes betreffen die Normen das Führungskonzept des Unternehmens (s. auch Tab. 8).

- So ist unter anderem festzulegen, wie die Leitungsstruktur des Krankenhauses - ob eher dezentral oder mehr zentral - ausgerichtet sein soll und ob und welche Entscheidungsbefugnisse unter Berücksichtigung der Führungskräfte- und Mitarbeitereigenschaften sowie der Eigenschaften der Entscheidungs-Situation von den Führungskräften an deren Mitarbeiter delegiert werden sollen (= Führungsorganisation).

- Es wird vereinbart, wie die Mitarbeiter an Entscheidungen, die ihre Vorgesetzten zu fällen haben, beteiligt werden sollen (= Führungsstil und Führungstechnik).

- Schließlich gilt es zu bestimmen, welche Bedeutung die Förderung von Mitarbeitern haben soll (= Entwicklungs- und Lernperspektive).

Wenn Entscheidungen der skizzierten Art, also im Sinne eines geänderten Wertekanons, gefällt und umgesetzt worden sind, kommt es darauf an, dafür zu sorgen, dass die Qualifikation der Führungskräfte und der Mitarbeiter so ausgerichtet wird, dass das neue Unternehmens- und Führungskonzept nachhaltig gelebt werden kann. Die Fähigkeiten der vorhandenen Führungskräfte und Mitarbeiter werden gegebenenfalls weiterentwickelt; bei der Rekrutierung neuer Führungskräfte und Mitarbeiter wird darauf geachtet, dass nur noch solche eingestellt werden, die bereit und imstande sind, dem neuen Unterneh- 
Tab. 8 Aspekte einer werteorientierten Personalarbeit

\begin{tabular}{|c|c|}
\hline Werte & Maßnahmen (Beispiele) im Sinne von Normen \\
\hline Selbstentfaltung & $\begin{array}{l}\text { durch Delegation von Verantwortung an Mitarbeiter für die Folgen ihrer } \\
\text { Entscheidungen }\end{array}$ \\
\hline Entwicklung von Demokratiebewusstsein & durch Beteiligung der Mitarbeiter an Entscheidungen \\
\hline Individualisierung & $\begin{array}{l}\text { durch Zuschreibung von Arbeitsergebnissen zu dem jeweils verantwortlichen } \\
\text { Mitarbeiter; Entkopplung von Arbeits- und Betriebszeit }\end{array}$ \\
\hline Karriereorientierung & $\begin{array}{l}\text { durch Personalentwicklung; Respektierung des Verhältnisses zwischen } \\
\text { Arbeitszeit und Freizeit und/oder ehrenamtlichem Engagement }\end{array}$ \\
\hline Selbstständigkeit & $\begin{array}{l}\text { durch Übertragung von Befugnissen an Mitarbeiter über die Festlegung } \\
\text { von Zielen und die zur Zielerreichung erforderlichen Mittel }\end{array}$ \\
\hline
\end{tabular}

mens- und Führungskonzept zur Wirksamkeit zu verhelfen.

Wer ist von den einschlägigen - im Sinne der oben erwähnten Anliegen der Mitarbeiter - strategischen Entscheidungen in welcher Weise betroffen?

\section{Stakeholder und deren Anliegen, bezogen auf Veränderungen des Wertesystems}

\section{Entscheidung der Geschäftsführung}

Die Geschäftsführung beschließt, ein an den Bedürfnissen der Führungskräfte und Mitarbeiter orientiertes FührungsKonzept zu entwickeln und einzuführen. Der Prozess der Entwicklung und Implementierung ist ausgerichtet an den Grundideen des Managements der sozialen Verantwortung.

\section{Stakeholder und Stakeholder-Anliegen}

\section{Patienten, Bevölkerung im Einzugsbereich des Krankenhauses und einweisende Ärzte}

Die Patienten, die Bevölkerung im Einzugsbereich des Krankenhauses sowie die einweisenden Ärzte werden davon profitieren, dass die Motivation der Mitarbeiter und damit deren Bereitschaft zu einer weiteren Steigerung ihrer Anstrengungen und in Folge davon der Behandlungs- und Servicequalität, auch im Sinne des uneingeschränkten Zugangs zu medizinischen Leistungen, verbessert werden. Bei der Entscheidung der Patienten, welches Krankenhaus sie im Bedarfsfall aufsuchen, und bei der Entscheidung der niedergelassenen Ärzte, in welches Krankenhaus sie ihre Patienten zur stationären Behandlung einweisen, wird das positive Verhalten der Mitarbeiter gegenüber Patienten, Angehörigen und einweisenden Ärzten eine nicht unerhebliche Rolle zu Gunsten des Krankenhauses spielen.

\section{Mitarbeiter}

Es gibt eine zunehmende Zahl von Mitarbeitern, die die erwähnten strukturellen Veränderungen in Entsprechung der von ihnen präferierten Werte erwarten und diese wenn sie realisiert werden - als Chance für eine weitere Verbesserung ihrer Arbeitssituation sehen. Sie werden die Geschäftsführung bei der Umsetzung eines neuen Führungskonzepts tatkräftig unterstützen.

Andere Mitarbeiter befürchten, dass sie den dann geänderten Anforderungen - den Mitarbeitern werden zusätzliche Entscheidungsbefugnisse übertragen und die Verantwortung für die sich aus den Entscheidungen ergebenden Folgen zugewiesen - nicht gerecht werden können und auch nicht über die notwendigen Potenziale für die Weiterentwicklung ihrer Fähigkeiten in Hinblick auf geänderte Anforderungen verfügen. Ihr Bedürfnis nach Sicherheit im Sinne von Bewältigenkönnen möglicherweise neuer Anforderungen sehen sie als eher weniger befriedigt und werden deshalb versuchen, die Änderung des Führungskonzepts zu verhindern.

\section{Führungskräfte}

Viele Führungskräfte werden ihre Mitarbeiter bei der Durchsetzung eines neuen Wertesystems und der Umsetzung des daraus entwickelten Führungskonzepts unterstützen. Sie werden dem Führungskonzept uneingeschränkt zur nachhaltigen Wirksamkeit verhelfen.

Es gibt aber immer noch Führungskräfte, denen es in Folge ihrer spezifischen Sozialisation schwer fällt, die Befriedigung der Bedürfnisse ihrer Mitarbeiter nach zum Beispiel mehr Selbstständigkeit und mehr Demokratie am Arbeitsplatz zu akzeptieren. Sie sehen deshalb auch nicht die Notwendigkeit, ihre Führungskompetenz im Sinne des neuen Wertesystems zu aktualisieren. Sie werden versuchen, die Entwicklung eines neuen Führungskonzepts zu verhindern. 


\section{Krankenkassen und Krankenhaus-Eigentümer}

Die Krankenkassen und der Krankenhaus-Eigentümer profitieren von dem Wertewandel und der Realisierung der daraus abgeleiteten Handlungsanweisungen. Die in Folge der Umsetzung des Werte-Wandelns in ein neues Führungskonzept zunehmende Zufriedenheit der Mitarbeiter mit ihrer Arbeitssituation führt zu einer weiteren Verbesserung der Behandlungs- und Servicequalität und des Preis-Leistungs-Verhältnisses. Die Attraktivität des Krankenhauses nimmt weiter zu. Die Inanspruchnahme steigt und damit die Auslastung der vorgehaltenen Kapazitäten. Das Krankenhaus ist für den an Schärfe zunehmenden Wettbewerb besser gerüstet und damit langfristig existenziell gesichert.

\subsubsection{Marktdynamik}

Gegenstände der Analyse von Veränderungen auf dem Krankenhaus-,,Markt“ sind vier Teilmärkte:

1. Auf dem ersten Teilmarkt bieten Krankenhäuser und andere Leistungsanbieter - zunehmend auf der Basis eines sich verschärfenden Wettbewerbs - medizinische Leistungen an. Patienten fragen diese Leistungen nach.

2. Auf einem zweiten Teilmarkt werden Krankenhäuser oder Teile von ihnen angeboten und nachgefragt.

3. Auf dem dritten Teilmarkt begegnen sich die Angebote diverser Waren und Dienstleistungen und die Nachfrage nach diesen durch Krankenhäuser.

4. Auf dem Arbeitsmarkt, dem vierten Teilmarkt, werden Arbeitsleistungen angeboten und durch Krankenhäuser nachgefragt.

Die weiteren Ausführungen beziehen sich vor allem auf die beiden ersten der vier Teilmärkte.

Der Wettbewerb auf dem Leistungsmarkt, dem ersten Teilmarkt des Krankenhaus,,marktes“, kann sich auf Grund einer ganzen Reihe gesetzlicher Vorschriften noch nicht so entfalten, wie es die Absicht des Gesetzgebers ist (Cassel, S. 15ff.). Dennoch:

- Krankenhäuser werden an andere, vielfach private Krankenhausträger verkauft, mit anderen Krankenhäusern fusioniert oder zur Gänze oder teilweise geschlossen ${ }^{38}$, wenn dem aktuellen Träger die Management- und/oder

38 Siehe zum Beispiel die Krankenhäuser in Salzgitter-Bad (2004), Herbolzheim (2006) sowie das St. Josef-Hospital in Oslberg (2004). finanziellen Ressourcen zum Weiterbetrieb des Krankenhauses fehlen.

- Krankenhäuser lagern medizinische und/oder nicht-medizinische Leistungsbereiche aus, um die Effizienz der Behandlung weiter steigern zu können und/oder

- ändern ihre Rechtsform mit dem Ziel, die Handlungsspielräume für das Management zu erweitern.

\section{Die Folgen sind:}

1. Es entstehen Regionen, innerhalb derer mehrere Krankenhäuser um die Gunst der Patienten werben. Dem kommt entgegen: In Folge der zunehmend leistungsfähigeren Verkehrssysteme nehmen Patienten künftig auch größere Wege in Kauf, um ein Krankenhaus ihrer Wahl aufsuchen zu können ${ }^{39}$.

2. Patienten müssen künftig längere Wege zum Erreichen des Krankenhauses in Kauf nehmen, als medizinisch verantwortbar und akzeptabel ist.

Nicht selten haben die genannten Maßnahmen Auswirkungen auf die Arbeitsverhältnisse von Mitarbeitern, die möglicherweise in ihrem Bestand oder hinsichtlich ihrer finanziellen Ausstattung gefährdet sind. Wenn ein kommunales Krankenhaus an einen privaten Träger veräußert wird, kommt hinzu, dass danach zwei unterschiedliche Kulturen aufeinander treffen. Das kommunale Krankenhaus ist - was für alle Krankenhäuser typisch ist - bedarfswirtschaftlich ausgerichtet. Bei einem Krankenhaus in privater Trägerschaft kommt ein erwerbswirtschaftliches Element - die Gewinnerzielungsabsicht - hinzu.

Von Entscheidungen der skizzierten Art sind Stakeholder des Krankenhauses wie folgt betroffen und prägen den Handlungsspielraum der $\mathrm{Ge}-$ schäftsführung:

39 Eine Studie der Koordinierungsstelle MedEcon Ruhr (Evans, S. 43f.) zeigt den Zusammenhang zwischen dem Grad der Spezialisierung und dem Einzugsbereich der Krankenhäuser, also der Bereitschaft der Patienten, gegebenenfalls auch größere Wege zwischen dem Wohnort und einem Krankenhaus zu akzeptieren. 


\section{Stakeholder und deren Anliegen, bezogen auf Veränderungen des Marktes}

\section{Entscheidung der Geschäftsführung}

Die Geschäftsführung des Krankenhaus-Verbundes $\mathrm{X}$ beschließt,

a) die Rechtsform zu ändern,

b) die Leistungsbereiche Gebäudereinigung und Speisenversorgung auszulagern und

c) eines der Verbund-Krankenhäuser an einen privaten Krankenhaus-Träger zu verkaufen.

\section{Stakeholder und Stakeholder-Anliegen}

\section{Patienten, Bevölkerung im Einzugsbereich des}

\section{Krankenhauses und niedergelassene Ärzte}

Patienten, deren Angehörige und die einweisenden Ärzte sind nicht selten mit Rechtsform-Änderungen, mit dem Outsourcing einzelner Leistungsbereiches des Krankenhauses und mit dem Verkauf von Krankenhäusern in öffentlichrechtlicher Trägerschaft an private Träger nicht einverstan$\operatorname{den}^{40}$. Dabei stehen im Regelfall die - meist unbegründeten - Bedenken im Vordergrund, private Krankenhausträger würden die marktgerechte Verzinsung des eingesetzten Kapitals zu Lasten der Interessen der Patienten und der Mitarbeiter gewichten und so bei ihren unternehmerischen Entscheidungen berücksichtigen; sie befürchten, dass unternehmerische Entscheidungen nicht mehr primär durch die Sorge um die Patienten geleitet wird (Kühn, S. 19ff.).

Die genannten Stakeholder befürchten im Falle des Auslagerns medizinischer und/oder nicht-medizinischer Leistungsbereiche, die Leistungen externer Dienstleistungsunternehmen wären weniger an den spezifischen Produktionsbedingungen eines Krankenhauses ausgerichtet als dies der Fall wäre, wenn die Serviceleistungen durch krankenhauseigene Servicebereiche erbracht würden.

Das Schließen von Krankenhäusern wird von den Patienten, den Bewohnern im Einzugsbereich des von der Schließung betroffenen Krankenhauses und von den niedergelassenen Ärzten abgelehnt. Sie sorgen sich, dass Patienten im Bedarfffall nicht rasch genug medizinische Hilfe erhalten können.

Begründet wird die Ablehnung der skizzierten Entwicklungen auch mit dem - nicht zutreffenden - Argument,

40 Siehe zum Beispiel das Volksbegehren gegen den Mehrheitsverkauf von Anteilen des Landesbetriebes Krankenhäuser Hamburg (LBK Hamburg) im Frühjahr 2003 und den Bürgerentscheid im Rottach-Inn-Kreis gegen den Verkauf des örtlichen Krankenhauses im Herbst 2009 der Staat würde sich damit - zumindest teilweise - seiner Verantwortung für die Sicherstellung der gesundheitlichen Versorgung der Bevölkerung entledigen.

\section{Mitarbeiter}

Die von den eingangs skizzierten Entwicklungen betroffenen Mitarbeiter werden in höchstem Maße verunsichert, weil sie nicht wissen, welche Anforderungen künftig an sie gestellt werden und ob sie diese bewältigen können. Sie werden mit anderen Unternehmenskulturen konfrontiert und müssen möglicherweise mit ihrer Kündigung und/ oder mit finanziellen Einbußen rechnen. Es ist ungewiss, ob sie ihrer Rolle als Agent der Patienten in dem gewohnten Maße gerecht werden können. Sie werden deshalb versuchen, den Status quo zu verfestigen.

\section{Führungskräfte}

Die Hauptlast zur Lösung der sich ergebenden Probleme liegt sicher bei den Führungskräften. Sie müssen die wegen vermeintlich schlechter werdender Arbeitsbedingungen entstehenden Motivations-Defizite durch geeignete Führungsleistungen ausgleichen. Sie sind gefordert, dazu beizutragen, die Einstellungen ihrer Mitarbeiter hinsichtlich ausschließlich bedarfswirtschaftlicher versus auch erwerbswirtschaftlicher Orientierung zu ändern.

Die Führungskräfte befürchten, dass ihr Bedürfnis nach Sicherheit im Sinne des Bewältigenkönnen neuer Anforderungen an ihre Führungsfähigkeit nicht mehr befriedigt wird. Sie werden deshalb versuchen, die skizzierten Veränderungen zu verhindern.

\section{Krankenkassen}

Krankenkassen versprechen sich als Ergebnis der unternehmerischen Entscheidungen - Rechtsformänderungen und/ oder Outsourcing und/oder Verkauf eines Krankenhauses in öffentlicher Trägerschaft an einen privaten Krankenhausträger - vor allem eine Verbesserung der Effizien $z^{41}$ der Behandlungs- und Serviceprozesse und damit des PreisLeistungs-Verhältnisses sowie die Steigerung der Qualität der Patientenbehandlung. Sie unterstützen deshalb die Bemühungen der Geschäftsführung.

Die Krankenkassen sind auf der anderen Seite nach den Bestimmungen des Krankenhausfinanzierungsgesetzes dazu verpflichtet, für die wirtschaftliche Sicherung der Krankenhäuser zu sorgen, um eine bedarfsgerechte Versorgung der Bevölkerung mit leistungsfähigen, eigenverantwortlich wirtschaftenden Krankenhäusern zu gewährleisten; sie sind deshalb auch an der Krankenhausplanung der

41 Andererseits zeigt eine Studie, dass Krankenhäuser in öffentlicher Trägerschaft effizienter arbeiten als private Krankenhäuser (Schreyögg, 0.S.). 
Bundesländer beteiligt (siehe z.B. \17 Abs. 1 Landeskrankenhausgesetz Berlin). Im Sinne dieser gesellschaftlichen Verpflichtung werden sie im Einzelfall prüfen, ob sie der Reduzierung von Behandlungskapazitäten oder deren Verlagerung von einer Region in eine andere zustimmen können.

\section{Krankenhaus-Eigentümer}

Wenn ein Krankenhaus veräußert oder mit einem anderen Krankenhaus fusioniert wird, geht die Initiative dazu im Regelfall - aus unterschiedlichen Gründen - von dem Eigentümer aus. Insofern stellt sich die Frage nach dessen Haltung zu diesem Vorgang nicht.

Hinsichtlich der Auslagerung von Leistungsbereichen wird der Eigentümer ähnlich und mit vergleichbaren Argumenten wie die Krankenkassen reagieren.

Eine Rechtsformänderung wird nicht selten durch die Geschäftsführung betrieben. Diese bedarf dazu der Zustimmung des Eigentümers. Dieser wird für diese Zustimmung gewonnen werden können, wenn primär mit der Einschränkung der Haftung für finanzielle Risiken argumentiert wird. Wenn dagegen die Leitungsbefugnisse zwischen der Geschäftsführung und den für die Aufsicht über das Krankenhaus zuständigen Instanzen des Krankenhaus-Eigentümers neu verteilt und damit die Eigenständigkeit der Geschäftsführung gestärkt werden soll, muss mit mehr oder weniger deutlichem Widerstand durch den Eigentümer gerechnet werden.

\subsection{Rahmenbedingungen}

\subsubsection{Soziodemografische Veränderungen}

\section{Einführung}

Europa ist der „ergrauende“ Kontinent (Kloimüller, S. 14). Während in den zehn Jahren vor 2004 der Anteil der bis 14-Jährigen an der Gesamtbevölkerung um etwa 2\%-Punkte abgenommen hat, stieg der Anteil der 65-79-Jährigen im gleichen Zeitraum um ca. 1.5\%-Punkte. Im Hinblick auf die Handlungssituation der Geschäftsführung bedeutet dieses folgendes:

1. Es muss damit gerechnet werden, dass der Anteil der über 65-jährigen Patienten an der Gesamtzahl der Patienten weiter zunimmt (Mehr als zwei Drittel der internistisch erkrankten Patienten sind derzeit älter als 65 Jahre [Steinhagen-Thiessen et al., S. 124].). Diese Entwicklung vollzieht sich in einer Zeit, in der „das Krankenhaus und das Pflegeheim ... Teil der Welt des altenverachtenden Alltags" sind (Rosenmayr, S. 197). Wer fit ist, gilt etwas.
Diese in unserer Gesellschaft immer noch vorherrschende Einstellung ist prägend für die Art und das Ausmaß der Zuwendung zu älteren und alten Patienten ${ }^{42}$.

2. Der Zahl der erwerbsfähigen Personen (im Alter zwischen 15 und 64 Jahren) sinkt bei gleichzeitig zunehmendem Bedarf an Beschäftigten in der Gesundheitswirtschaft (s. Abb. 26); die Belegschaften der Krankenhäuser werden älter.

Die soziodemografischen Veränderungen gehören zu jenen Einflussfaktoren, auf die die Geschäftsführung - im Gegensatz zum Beispiel zu dem Wertewandel - nur reagieren kann bzw. mittel- und langfristig reagieren muss, wenn sie eine ausreichend hohe Inanspruchnahme des Krankenhauses dadurch sichern und gewährleisten will, dass die Leistungsfähigkeit der Belegschaft für die Bewältigung der möglicherweise steigenden Anforderungen hoch genug bleibt.

\section{Patienten werden älter}

Die aktuelle Situation alter Patienten im Krankenhaus lässt sich wie folgt charakterisieren:

Das geltende Finanzierungsrecht, das pauschalierende Entgeltsystem, beinhaltet Anreize zur Risikoselektion durch das Abkürzen der Behandlungsdauer (Schulz-Nieswandt, S. 59). Dieses hat immer wieder die Zuweisung der Patienten in klinisch instabilen Zuständen $\mathrm{zu}$ anderen Leistungsanbietern (ambulante Behandlung, Rehabilitation, Langzeitpflege) oder die erneute Aufnahme in die stationäre Behandlung zur Folge (Schulz-Nieswandt, S. 6o). Weil vor allem alte Patienten große Schwierigkeiten haben, sich in dem komplexen Gesundheitssystem allein zurecht zu finden, wird deren Bedürfnis nach transsektoraler Integration (Schulz-Nieswandt, S. 59) von zentraler Bedeutung werden.

Durch ineinander greifende transparente Versorgungsketten werden gerade alte, multimorbide und chronisch kranke Menschen im System aufgefangen; sie können länger selbstständig ihren Alltag gestalten. Viele der Patienten, die ansonsten in die stationäre Behandlung aufgenommen werden müssten, können im Rahmen

42 Nachfolgend wird zu Gunsten der besseren Lesbarkeit zusammenfassend von alten Patienten/Menschen gesprochen. Es sind da mit immer beide Lebensaltersgruppen gemeint. 




Abb. 26 Die Entwicklung der Erwerbstätigen in Deutschland insgesamt und in der Gesundheitswirtschaft (in Anlehnung an Henke et al., S. 30)

eines geeigneten Integrationsmodells ambulant behandelt werden. Die formalen Voraussetzungen dafür hat der Gesetzgeber mittels des $\$$ 140a-h Sozialgesetzbuch (SCB) V geschaffen; es ist zu beobachten, dass erste Integrationsmodelle mit dem Ziel, die skizzierten nachteiligen Folgen des neuen Finanzierungsrechts zu vermeiden, entwickelt worden sind. Die Initiative dazu geht vor allem von den Krankenhäusern aus.

Das Akut-Krankenhaus definiert sich herkömmlicherweise durch das Ziel, Krankheiten zu heilen und die Gesundheit der Patienten voll wiederherzustellen. Diagnostik und Therapie bestimmen deshalb die innere Struktur und die Organisation des Krankenhauses (Rosenmayr, S. 198). Das Ziel der Behandlung alter Menschen ist dagegen nicht selten fokussiert auf die Wiederherstellung größtmöglicher Selbstständigkeit trotz eventuell bleibender Behinderungen. Das in diesem Zusammenhang notwendige aktive Bemühen um Aufrechterhalten der Kompetenz und der Kontrolle im Alter (Marbach, S. 43) werden aber im Krankenhaus ebenso wie die Unterstützung der alten Menschen bei der Bewältigung der Aktivitäten des täglichen Lebens noch immer als ein Randproblem gesehen. Häufig sind weder die Arbeitsweise noch die Ausstattung der
Krankenhäuser für die Realisierung dieses Behandlungszieles geeignet (Steinhagen-Thiessen et al., S. 140).

Die Diagnostik alter, vielfach multimorbider Patienten ist häufig sehr aufwändig. Um die Einrichtungen des Krankenhauses effizient nutzen und um die Dauer des Aufenthaltes der Patienten im Krankenhaus minimieren zu können, erfolgt die Diagnostik - auch bei den alten Patienten nach einem Plan, der wenig Erholungspausen zwischen den verschiedenen Untersuchungen zulässt und damit gerade für die alten Patienten besonders belastend ist. Hinzu kommt, dass der gewohnte Tagesrhythmus eines alten Menschen ein anderer ist als der eines jüngeren; die vielfach ausschließlich anhand von Effizienz-Kriterien gestalteten Arbeitsabläufe kommen diesem Bedürfnis nur selten entgegen.

Viele der alten Patienten sind neben ihrer aktuellen Erkrankung körperlich behindert. Die baulichen und technischen Bedingungen der Krankenhäuser sind sehr häufig darauf noch nicht ausgerichtet.

Die Pharmatherapie ist im Regelfall für jüngere Patienten konzipiert und berücksichtigt nicht das im Alter geschwächte Adaptionsvermögen. Die Wirkung ist deshalb nicht selten nicht die 
gewünschte; es treten gehäuft Nebenwirkungen auf (Coper; Schulze, S. 204).

Es wird auch einer anderen Ansprache bedürfen, um alte Patienten angemessen aufklären zu können. Um die Integration alter Patienten in den Behandlungsprozess im Sinne der so genannten Ko-Produktionsthese realisieren zu können, ist ein anderer Umgang mit dem Patienten erforderlich als dies bei einem 30-Jährigen der Fall ist.

Zusammenfassend lässt sich festhalten, dass die Krankenhäuser

- in die Realisierung geeigneter Integrationsmodelle,

- in die Professionalisierung der Geschäftsführung und aller Mitarbeiter, die Behandlung alter Patienten betreffend,

- in die Veränderung der Einstellung der Ceschäftsführung und aller Mitarbeiter gegenüber den Bedürfnissen alter Patienten sowie

- in die Anpassung der Arbeitsabläufe und der baulichen und technischen Ausstattung

investieren müssen.

Von Entscheidungen der skizzierten Art sind Stakeholder des Krankenhauses wie folgt betroffen.

\section{Stakeholder und deren Anliegen, bezogen auf Veränderungen der Altersstruktur der Patienten}

\section{Entscheidung der Geschäftsführung}

Die Geschäftsführung beschließt, zusammen mit niedergelassenen Ärzten des Einzugsgebietes ein Medizinisches Versorgungszentrum (MVZ) zu gründen, das sich insbesondere der Versorgung alter Patienten widmet.

Es wird ein Weiterbildungsprogramm, die Behandlung alter Menschen betreffend, entwickelt und eingeführt. Im Verlauf eines Zeitraumes von $x$ Monaten sollen alle Führungskräfte und Mitarbeiter an diesem Programm teilgenommen haben. Für danach eingestellte Führungskräfte und Mitarbeiter wird das Programm einmal pro Halbjahr angeboten.

Eine Arbeitsgruppe wird Vorschläge erarbeiten für die auf die Behandlung alter Menschen bezogene Weiterentwicklung der Arbeitsabläufe sowie der Anpassung der baulichen und technischen Ausstattung.

\section{Stakeholder und Stakeholder-Anliegen}

\section{Patienten, Bevölkerung im Einzugsbereich des} Krankenhauses und niedergelassene Ärzte

Die Patienten sowie die einweisenden Ärzte werden vor allem jene Krankenhäuser verstärkt in Anspruch nehmen, die Bestandteil eines entsprechend den Bedürfnissen alter
Patienten gestalteten Versorgungsmodells sind; dieses vor allem auch deshalb, weil sie sich dadurch die Stabilisierung der Behandlung auch über die stationäre Behandlung hinaus versprechen. Die Menschen im Einzugsbereich werden sich je nach der Art des angebotenen Integrationsmodells dort einschreiben und somit eine dauerhafte Bindung zu dem Krankenhaus und den anderen in dem Integrationsmodell zusammen geschlossenen Leistungsanbietern begründen.

Mit der an den Bedürfnissen alter Patienten orientierten Weiterentwicklung der personellen, strukturellen, organisatorischen und technischen krankenhausinternen Behandlungs-Bedingungen wird die Geschäftsführung einen entscheidenden Beitrag zur Lösung eines Profilierungsproblems und damit zum Bestehen des Krankenhauses im Wettbewerb leisten. Die Patienten, die Bevölkerung im Einzugsbereich des Krankenhauses und die niedergelassenen Ärzte werden die Investitionen des Krankenhauses durch zunehmende Inanspruchnahme und durch dauerhafte Bindung an das Krankenhaus honorieren.

\section{Mitarbeiter}

Die Mehrzahl der Mitarbeiter begrüßt die genannten Veränderungen, weil sie damit ihre Agenten-Rolle auch gegenüber alten Patienten ohne Abstriche wahrnehmen können. Sie unterstützen die Geschäftsführung bei der Umsetzung der geplanten Veränderungen auch deshalb, weil sie damit eine Steigerung der Reputation des Krankenhauses erwarten und in Folge davon für sich selbst eine Zunahme ihrer Wertschätzung durch ihr soziales Umfeld.

Auf der anderen Seite wird es Mitarbeiter geben, die nicht sicher sind, ob sie der durch die Geschäftsführung verlangten Änderung ihrer Einstellung gegenüber alten Menschen und der im Sinne der spezifischen Behandlung alter Patienten erforderlichen Vervollkommnung ihrer Kompetenz werden folgen können. Ihr Bedürfnis nach Sicherheit, nach Bewältigenkönnen der neuen Anforderungen, wird möglicherweise nicht mehr zureichend befriedigt. Sie werden deshalb das Vorhaben der Geschäftsführung eher mit Zurückhaltung begleiten.

Im Übrigen wird auf die Reaktion der Mitarbeiter im Zusammenhang mit Leistungsanbieter übergreifenden organisatorischen Veränderungen verwiesen. Diese können auch hinsichtlich der hier skizzierten, aus anderen Gründen initiierten Leistungsanbieter übergreifenden organisatorischen Veränderungen eine Rolle spielen.

\section{Führungskräfte}

Die Führungskräfte werden grundsätzlich wie ihre Mitarbeiter reagieren.

Ihre Reserviertheit gegenüber den skizzierten Veränderungen wird wegen der von innen erwarteten Motivation ihrer Mitarbeiter zur Veränderung ihrer Einstellung und 
zum Ausbau ihrer Kompetenzen verstärkt. Sie sind nicht sicher, ob sie über die dafür notwendigen Entwicklungspotenziale verfügen. Ihr Bedürfnis nach Sicherheit, nach Bewältigenkönnen der neuen Anforderungen, wird auch deshalb eher nur eingeschränkt befriedigt. Sie werden auch deshalb die Pläne der Geschäftsführung eher nicht unterstützen.

\section{Krankenkassen}

Die Krankenkassen müssen angesichts der Zunahme der altersbedingten Schweregrade der Erkrankungen und Multimorbidität mit einem steigenden Case-Mix-Index und damit insgesamt mit steigenden Krankenhaus-Budgets rechnen ${ }^{43}$. Die einzelne Krankenkasse muss deshalb aber nicht befürchten, wegen eines überproportional höheren Anteils alter Patienten in der stationären Behandlung überproportional höhere Behandlungskosten ohne Ausgleich tragen zu müssen. Der morbiditätsorientierte Risikostrukturausgleich sorgt zwischen den Krankenkassen zu einem Ausgleich der zu erwartenden höheren Belastungen durch höhere Zuweisungen aus dem Gesundheitsfonds. Insofern werden die Krankenkassen die Geschäftsführung bei der Realisierung von Maßnahmen mit dem Ziel, die oben skizzierten Defizite zu beseitigen, unterstützen - auch dann, wenn damit gerechnet werden muss, dass in Folge einer Behandlung, die besser auf die Bedürfnisse alter Patienten abgestimmt ist, Letztere zunehmend dieses Krankenhaus aufsuchen und ein höheres Budget bedingen werden.

Dieses gilt insbesondere dann, wenn die Geschäftsführung durch die ergänzende Etablierung geeigneter Integrationsmodelle dafür sorgt, dass Patienten im Bedarfsfall möglichst, wenn medizinisch vertretbar, ambulant und weniger stationär behandelt werden.

\section{Eigentümer}

Es kann damit gerechnet werden, dass das Entgeltsystem hinsichtlich der altersbedingten Schweregrade und Multimorbidität künftig besser als bisher ausdifferenziert sein wird. Dennoch befürchtet der Eigentümer, dass die Kosten stärker steigen werden als die Erlöse. Er wird deshalb der Investition in die Verbesserung der Behandlung alter Patienten und damit in die Verbesserung der Attraktivität des Krankenhauses für gerade jene Patientengruppe eher reserviert gegenüber stehen.

Der Eigentümer unterstützt die Entwicklung und Implementation von Integrationsmodellen. Er erhofft sich

43 Das ist allerdings umstritten. Der Anteil der älteren und alten Menschen an der Bevölkerung wird zwar zunehmen. Es zeigt sich allerdings, dass sich eine zunehmende Zahl älter werdender Menschen ihre gute Gesundheit auch bis in das höhere Alter hinein bewahren kann. dadurch die Entlastung des stationären Bereichs von besonders aufwändig zu behandelnden Patienten, indem diese, wenn medizinisch vertretbar, ambulant behandelt werden. Er verspricht sich außerdem für das Krankenhaus einen Zugewinn an Reputation, weil dem Anliegen der Patienten nach Stabilisierung der Behandlung auch über die stationäre Behandlung hinaus entsprochen wird, und damit mit einer verstärkten Inanspruchnahme, zusätzlichen Erlösen und der effizienteren Auslastung der vorhandenen Ressourcen gerechnet werden kann.

\section{Krankenhaus-Belegschaften werden älter}

Die Entwicklung auf dem Arbeitsmarkt lässt erwarten, dass es immer schwieriger werden wird, eine ausreichend große Zahl von gut qualifizierten und motivierten, vor allem auch jungen Mitarbeitern für eine Tätigkeit im Krankenhaus zu gewinnen und dort über eine längere Zeit hinweg zu binden. Der Wettbewerb auf dem Arbeitsmarkt - der War for talents - nimmt an Schärfe zu.

\section{Hinzu kommt:}

- Einzelne Berufsgruppen, wie zum Beispiel die Gesundheits- und Krankenpfleger, sind besonderen Belastungen ausgesetzt. So weist das Statistische Bundesamt in einer Studie, veröffentlicht am 18. August 2009, darauf hin: Steigende Patientenzahlen und Verkürzungen der Verweildauer in Krankenhäusern führen bei gleichzeitigem Pflegepersonalabbau zu einer Arbeitsverdichtung. Nach den Ergebnissen des Mikrozensus 2007 sind Gesundheits- und Krankenpflegerinnen/-pfleger bezogen auf Schichtarbeit, Wochenend- und Feiertagsarbeit sowie Nachtarbeit besonders stark belastet. Es wurde weiter deutlich, dass diese Berufsgruppe besonders häufig unter arbeitsbedingten Gesundheitsproblemen leidet. Zudem ist ihre Tätigkeit im besonderen Ausmaß durch schwierige Körperhaltungen, Bewegungsabläufe oder Hantieren mit schweren Lasten sowie Zeitdruck und Arbeitsüberlastung gekennzeichnet.

- Die Schere zwischen den Anforderungen an die Krankenhaus-Belegschaften und deren Leistungsfähigkeit öffnet sich immer mehr.

- Die Qualität der Beschäftigungsverhältnisse wird geringer (Dathe et al., S. 3).

- Die Personalausstattung entspricht in Folge der Bindung der Personalkosten an die 
geringer werdenden Erlöse immer häufiger nicht mehr dem Prinzip der Bedarfsgerechtigkeit (zu Bedarfsgerechtigkeit s. Naegler et al., S. 83f.). Finanzierte Stellen können wegen des sich abzeichnenden Mangels an Bewerbern - dieses gilt derzeit insbesondere für Ärzte - vermehrt nicht oder nicht zeitgerecht besetzt werden.

- Die Qualifikation der Bewerber entspricht zunehmend nicht den Anforderungen der zu besetzenden Stellen.

- Die Krankenhaus-Belegschaften werden älter.

Umso mehr kommt es darauf an, die Arbeitsbedingungen in den Krankenhäusern so zu gestalten, dass die Leistungsfähigkeit der Mitarbeiter bis $\mathrm{zu}$ ihrem altersbedingten Ausscheiden aus dem Berufsleben möglichst nicht geringer wird oder dass der Prozess der LeistungsfähigkeitsMinderung zumindest verlangsamt wird. Dabei werden unter den Arbeitsbedingungen die Anforderungen, die sich aus der wahrzunehmenden Aufgabe ergeben, die Arbeitsumgebung, die Organisation der Arbeit (einschließlich Führungskonzept und Arbeitszeitgestaltung) sowie die Entlohnung der Arbeit verstanden. Zu den Arbeitsbedingungen zählt schließlich auch die Unterstützung der Mitarbeiter durch den Arbeitgeber, damit die
Mitarbeiter ihren Verpflichtungen gegenüber ihren Familien nachkommen können. Welche Faktoren es im Einzelnen sind, die zu der vorzeitigen Minderung der Leistungsfähigkeit der Mitarbeiter und möglicherweise auch zu deren vorzeitigen Ausscheiden aus dem Arbeitsprozess beitragen können und die deshalb Ansätze für die Korrektur der Arbeitsbedingungen sein sollten, ist der Tabelle $9 \mathrm{zu}$ entnehmen.

Zu den Maßnahmen, die dem Erhalt der Leistungsfähigkeit älter werdender Mitarbeiter dienen, zählen auch Maßnahmen der Personalentwicklung. Diese sind notwendig, damit auch alte Mitarbeiter über die dem neuesten Stand der Wissenschaft und Praxis entsprechenden Fähigkeiten und Fertigkeiten verfügen. Sie sind damit im Stande, weiterhin besonders gute Leistungen zu erbringen; sie können im internen Wettbewerb um Anerkennung und Wertschätzung mit den jüngeren Mitarbeitern bestehen. Personalentwicklungs-Maßnahmen sind aber auch erforderlich, um die älter werdenden Mitarbeiter in die Lage zu versetzen, ihre Aufgaben auf der Crundlage geänderter Arbeitsbedingungen erfolgreich wahrzunehmen.

Der Spielraum der Geschäftsführung für Entscheidungen im Sinne der alternsgerechten $\mathrm{Ge}$ staltung der Arbeitsbedingungen wird unter an-

Tab. 9 Faktoren, die ein erhöhtes Risiko für die - vor allem älteren - Arbeitnehmer bedeuten (in Anlehnung an Ilmarinen, S. 28f.)

\begin{tabular}{|c|c|c|}
\hline $\begin{array}{l}\text { Zu hohe physische } \\
\text { Arbeitsanforderungen }\end{array}$ & $\begin{array}{l}\text { Belastende und gefährliche } \\
\text { Arbeitsumgebung }\end{array}$ & Mangelhaft organisierte Arbeit \\
\hline statische Muskelarbeit & $\begin{array}{l}\text { schmutzige oder nasse } \\
\text { Arbeitsumgebung }\end{array}$ & Rollenkonflikte \\
\hline hoher Krafteinsatz & Unfallrisiko & Kontrollieren und Angreifen \\
\hline Heben und Tragen & Hitzearbeit & Angst vor Fehlleistungen \\
\hline sonstige Spitzenbelastungen & Kältearbeit & Mangel an Freiheitsgraden \\
\hline repetetive Arbeit & rasche Temperaturänderungen & Zeitdruck \\
\hline \multirow[t]{4}{*}{$\begin{array}{l}\text { gleichzeitig gebeugte und } \\
\text { gedrehte Körperhaltung }\end{array}$} & & $\begin{array}{l}\text { Mangel an Einflussmöglichkeiten auf die } \\
\text { eigene Arbeit }\end{array}$ \\
\hline & & Mangel an beruflicher Perspektive \\
\hline & & mangelhafte Anerkennung durch den Vorgesetzten \\
\hline & & $\begin{array}{l}\text { unzureichende Vereinbarkeit zwischen Beruf } \\
\text { und Familie }^{1}\end{array}$ \\
\hline
\end{tabular}


derem von den folgenden Stakeholder-Anliegen geprägt.

\section{Stakeholder und deren Anliegen, bezogen auf Veränderungen der Altersstruktur der Krankenhaus-Belegschaften}

\section{Entscheidung der Geschäftsführung}

Die Geschäftsführung beschließt, die Arbeitsbedingungen alternsgerecht weiter zu entwickeln.

\section{Stakeholder und Stakeholder-Anliegen}

\section{Patienten, Bevölkerung im Einzugsbereich des Krankenhauses und niedergelassene Ärzte}

Patienten, die im Einzugsbereich des Krankenhauses lebenden Menschen und die einweisenden Ärzte werden es honorieren, wenn die Geschäftsführung ihren älter werdenden Belegschaften Arbeitsbedingungen anbietet, die das alternsbedingte Absinken der Leistungsfähigkeit verlangsamen und die verhindern können, dass älter werdende Mitarbeiter vorzeitig aus dem Arbeitsprozess ausscheiden müssen. Sie begrüßen es deshalb, weil sie es zu schätzen wissen, dass sie von Experten versorgt werden, die neben ihrem Fachwissen auch über eine langjährige Berufs- und Lebenserfahrung verfügen und auf dieser Grundlage die spezifischen Bedürfnisse kranker und alter Menschen sehr viel besser befriedigen können als dies die jungen Mitarbeiter, die zwar die modernen medizinischen Methoden beherrschen, leisten können.

\section{Mitarbeiter}

Die Mitarbeiter begrüßen einerseits die Ausrichtung der Arbeitsbedingungen an ihre sich altersgemäß ändernde Leistungsfähigkeit. Ihre Bedürfnisse nach Sicherheit im Sinne des Bewältigens ihrer Aufgaben - vor allem als Agent ihrer Patienten - auch noch im höheren Alter und des Erhalts ihres bisherigen Arbeitsplatzes, nach Zugehörigkeit zu der gewohnten Arbeitsgruppe, nach Wertschätzung und nach Entfaltung ihrer Persönlichkeit auch noch im höheren Alter werden damit befriedigt.

Sie befürchten andererseits, nicht imstande zu sein, den geänderten Anforderungen gerecht werden zu können, und nicht über die Entwicklungspotenziale für die erfolgreiche Anpassung ihrer Fähigkeiten an die neuen Anforderungen zu verfügen. Weil aber die Gefahr besteht, dass es der Geschäftsführung nicht gelingen könnte, eine ausreichend hohe Zahl qualifizierter Mitarbeiter für eine Tätigkeit im Krankenhaus zu gewinnen und dort über eine längere Zeit zu binden, werden sie ihre Bedenken überwinden und alle Anstrengungen unternehmen, um ihre Kompetenzen entsprechend den geänderten Anforderungen ausbauen zu können.

\section{Führungskräfte}

Die Aufgabe der Führungskräfte wird es sein, die Entscheidungen der Geschäftsführung, die geänderten Arbeitsbedingungen betreffend, zu realisieren. Sie werden die Geschäftsführung in diesem Sinne unterstützen, obwohl dies gilt vor allem für die älteren von ihnen - es ihnen nicht leicht fallen wird, ein modernes Führungskonzept, das den Bedürfnissen der Mitarbeiter nach mehr Demokratie am Arbeitsplatz, Wertschätzung, Selbstverwirklichung und Selbstentfaltung entgegen kommt, umzusetzen.

\section{Krankenkassen; Eigentümer}

Die dauerhafte Existenz des Krankenhauses hängt vor allem von der Verfügbarkeit über eine ausreichende Zahl gut qualifizierter und motivierter Mitarbeiter ab. Deshalb werden die Krankenkassen und der Eigentümer des Krankenhauses die Geschäftsführung bei deren Investitionen in alternsgerechte Arbeitsbedingungen unterstützen - auch wenn dadurch kurzfristig die Kosten steigen werden und der Eigentümer seine Renditeerwartungen vorübergehend reduzieren muss.

\subsubsection{Finanzielle Rahmenbedingungen}

Wenn anschließend von finanziellen Rahmenbedingungen gesprochen wird, dann wird damit ausschließlich die Seite der Mittelherkunft zum Thema gemacht. Die Seite der Mittelverwendung - also die Beantwortung der Frage, welche Handlungen mit welchen Folgen Kostenveränderungen auslösen - bleibt außen vor.

Es bietet sich an, in diesem Sinne von zwei Arten von finanziellen Rahmenbedingungen auszugehen (Naegler et al., S. 12f.), weil die Entscheidungen, die von Veränderungen des finanziellen Rahmens für die Krankenhausleitungsarbeit ausgelöst werden, für die einzelnen Stakeholder - in Abhängigkeit von der Art der Rahmenbedingungen - unterschiedliche Folgen haben können. So lassen sich

- finanzielle Rahmenbedingungen struktureller Art - wie zum Beispiel der Ersatz des über eine lange Zeit hinweg praktizierten durch ein pauschalierendes Entgelt-System (zu dem DRG-System s. Behrends, S. $79 f f$.$) - von$

- finanziellen Rahmenbedingungen materieller Art - wie zum Beispiel die Änderung des für die Bezahlung der Krankenhausleistungen durch die Krankenkassen bzw. Patienten maßgeblichen Basisfallwert unterscheiden. 
Das bisher (bis 1992) zu praktizierende System zur Abrechnung der Krankenhausleistungen war, weil es Elemente eines Selbstkostendeckungsprinzips beinhaltete und von einer Struktur ausging, die nicht auf dem Behandlungsprozess und einem einheitlichen Prozess-Endprodukt basiert, sondern Teilprodukte abrechnete, nicht dazu geeignet, eine hohe Behandlungs- und Servicequalität sowie ein günstiges Preis-Leistungs-Verhältnis zu belohnen. Es hat zu der Entwicklung von Management-Verhalten und -Praktiken beigetragen, die die negativen systemimmanenten Tendenzen noch verstärkten.

Infolge der deshalb notwendig gewordenen neuen gesetzgeberischen Vorgaben $(\$ 17 \mathrm{~b}$ Krankenhausfinanzierungsgesetz [KHG]) ändern sich die Rahmenbedingungen sowohl für das Gesundheitswesen insgesamt als auch die für die einzelnen Krankenhäuser grundlegend. Die Abrechnung der Krankenhausleistungen auf der Basis eines pauschalierenden Entgeltsystems stellt die Geschäftsführung der Einrichtungen im Gesundheitswesen im allgemeinen und das der Krankenhäuser im besonderen vor neue BetriebsführungsHerausforderungen.

Es wurde erwartet und lässt sich ansatzweise bereits beobachten, dass die neuen formalen Rahmenbedingungen zu einer Umorientierung von der reinen Aufgabenerfüllung und vom Denken in Zuständigkeiten hin zu der weiter verstärkten Ausrichtung der Behandlungs-, Support- und Betriebsführungsprozesse an der Befriedigung von Patienten- bzw. Kundenbedürfnissen beitragen werden. Die Interessen der Patienten und der sie einweisenden Ärzte einerseits und die der Geschäftsführung andererseits hinsichtlich der Gestaltung der Verweildauer sind weitgehend gleichgerichtet: Während die Patienten und die sie einweisenden Ärzte schon immer an einem in der Regel möglichst kurzen Aufenthalt der Patienten im Krankenhaus interessiert waren, hat nunmehr auch die Geschäftsführung nicht mehr das Interesse, die Patienten aus Gründen der Erlösmaximierung möglichst lange im Krankenhaus zu behalten, und leistet mit dieser Neuorientierung einen Beitrag gleichermaßen zu der Steigerung der Patientenzufriedenheit sowie der Behandlungseffektivität und -effizienz.

Es stellt sich künftig nicht mehr die Frage: „Wie viele Tage wird der Patient voraussichtlich im Krankenhaus verbringen müssen?", sondern: „Wie viel Zeit darf die Behandlung in Anspruch nehmen und wie viele Tage darf der Patient des- halb höchstens im Krankenhaus bleiben?" Abweichungen nach oben und unten von einer je Krankheitsbild definierten Length of Stay (LOS) sind in dem neuen Abrechnungssystem vorgesehen und lösen von der DRG-Pauschale abweichende Vergütungen aus.

Die Abrechnung mithilfe von DRC's wird nicht nur zu einer grundlegend neuen Zielsetzung, dem Target Timing, führen; das Target Timing zielt auf eine zeitorientierte Koordination aller Leistungsbereiche des Krankenhauses ab. Sie wird ferner zu einer fallbezogenen Organisation des Krankenhauses anregen und damit dem Geschäftsprozessmanagement als Instrument der Geschäftsführung zu mehr Bedeutung als bisher verhelfen.

Viele Experten erwarten, dass die Erlöse in einer ganzen Reihe von Krankenhäusern künftig möglicherweise nicht mehr ausreichen werden, die Kosten der stationären Patientenversorgung zu decken. Die Konkurrenz zwischen den Bedürfnissen der Patienten nach einer den aktuellen medizinischen Erkenntnissen entsprechenden Versorgung, nach einer evidence-based-medicine, den Bedürfnissen der Mitarbeiter nach Arbeitsplatzsicherung und nach einer angemessenen Entlohnung ihrer Tätigkeit, den Forderungen der Politik nach Stabilisierung der Krankenkassen-Beiträge und den Erwartungen der Krankenhaus-Eigentümer nach einer angemessenen Verzinsung des von ihnen zur Verfügung gestellten Kapitals nimmt an Schärfe zu.

Die Veränderungen der finanziellen Rahmenbedingungen struktureller Art sind durch einschlägige Gesetze vorgegeben und müssen von der Geschäftsführung umgesetzt werden. Gleiches gilt für die Relativgewichte (s. Behrends, S. 79), die zusammen mit dem Basisfallwert die Obergrenze für die Verursachung der Kosten und damit für den Ressourceneinsatz markieren. Einen allerdings sehr engen - Handlungsspielraum hat die Geschäftsführung in Bezug auf die Gestaltung des Basisfallwertes.

Die Konsequenzen, die die Geschäftsführungen in Folge der Veränderungen der finanziellen Rahmenbedingungen materieller Art hinsichtlich der Betriebsführung ziehen werden, sind in den einzelnen Krankenhäusern unterschiedlich. Sie reichen von

1. Nichtstun und darauf hoffen, dass sich einiges von alleine zum Besseren wendet über

2. den Versuch der Erlösmaximierung durch entsprechende Kodier-Anstrengungen,

3. die Rationierung von Leistungen bis zu 
4. der Standardisierung der Behandlungsprozesse und der für die Behandlung einzusetzenden Ressourcen sowie dem Schaffen der dafür notwendigen, am Behandlungsprozess ausgerichteten Strukturen.

Welche Strategien von dem Management in den etwa 2.200 Krankenhäusern in Deutschland verfolgt werden, ist noch nicht ausgemacht. Viele werden es mit den Strategien 1 bis 3 versuchen, bis es sich herausstellt, dass sich damit ein gutes Gesamtergebnis für alle Beteiligte und Betroffene im Sinne einer effizienten Patientenbehandlung auf hohem qualitativem Niveau nicht erreichen lässt. Die unternehmerisch denkenden und handelnden Geschäftsführungen werden dagegen auf die Strategie 4 setzen und damit trotz weniger Ressourceneinsatz - damit sind vor allem die personellen Ressourcen gemeint - eine bessere Krankenbehandlung ermöglichen (Hildebrand 2008, S. 5). Die Reduzierung des Ressourceneinsatzes wird aufgefangen durch die Verbesserung der Strukturen und der Arbeitsabläufe und vor allem durch die Konzentration auf das medizinisch wirklich Notwendige. Die Geschäftsführung macht deutlich, dass sie der Realisierung einer an evidence-based-medicine und ethischen Grundsätzen orientierten Behandlung mit einem nachweislich guten Behandlungsergebnis verpflichtet ist.

In der Annahme, dass sich die Strategie 4 letztlich durchsetzen wird, ist auch diese nur Gegenstand der weiteren Untersuchung. Es ist zu prüfen, welche Bedürfnisse der verschiedenen Stakeholder betroffen sind, wenn sich die Geschäftsführung für ein - auch was dessen Umsetzung anbelangt - ambitioniertes Programm entscheidet. Die weiteren Betrachtungen stellen die Reaktionen auf die Standardisierung der Prozesse und des Ressourceneinsatzes in den Vordergrund (s. folgenden Textabschnitt). Die Reaktionen auf die in Folge der geänderten Finanzierungsregeln zu erwartenden Veränderung der Aufbauorganisation sind Gegenstand des Kapitels 4.3.4, Krankenhausinterne Veränderungen.

\section{Stakeholder und deren Anliegen, bezogen auf Veränderungen der Finanzierung}

\section{Entscheidung der Geschäftsführung}

Standardisierung der Behandlungsprozesse und der für die Behandlung einzusetzenden Ressourcen sowie Schaffen der am Behandlungsprozess ausgerichteten Strukturen.

\section{Stakeholder und Stakeholder-Anliegen}

\section{Patienten, Bevölkerung im Einzugsbereich des Krankenhauses und niedergelassene Ärzte}

Ein Teil der „Kunden“ des Krankenhauses wird diese Strategie begrüßen und im Bedarfsfall mit der Präferierung des Krankenhauses honorieren. Dabei gehen sie davon aus, dass ihr Bedürfnis nach im Bedarfsfall uneingeschränktem Zugang zu medizinischen Leistungen sowie nach hoher Behandlungssicherheit und -qualität, wobei mit Letzterer vor allem auch die Reduzierung der Behandlungsdauer auf das medizinisch Notwendige gemeint ist, mehr als bisher befriedigt wird.

Eine zweite Gruppe von „Kunden“ wird - nicht selten beeinflusst durch kritische Stimmen aus dem Kreis der Mitarbeiter und der Personalvertretung und meist unbegründet - befürchten, dass die Reduzierung des Ressourceneinsatzes zu einer Verringerung der Behandlungssicherheit und -qualität führen wird. Sie werden deshalb versuchen, das Umsetzen der Pläne der Geschäftsführung zu verhindern.

\section{Mitarbeiter}

Die Veränderung der Strukturen und der Arbeitsabläufe, verbunden mit dem konsequenten Verzicht auf nicht Notwendiges lösen bei den Mitarbeitern mehrere Arten von Reaktionen aus. Diese Reaktionen sind geprägt durch die Rolle der Mitarbeiter als Agenten der Patienten und ihren beiden Individualzielen - bestmögliche Behandlung der Patienten und Befriedigung ihrer persönlichen Bedürfnisse (Naegler et al., S. 267) -, deren Realisierung sie mit der Wahrnehmung ihrer Aufgaben verfolgen. Je nachdem, welches dieser Individualziele primär das Verhalten der Mitarbeiter steuert, werden diese unterschiedlich auf die Pläne der Geschäftsführung reagieren:

- Eine erste Gruppe von Mitarbeitern sieht mit den geplanten Maßnahmen die Befriedigung ihres Bedürfnisses nach Sicherheit gefährdet. Sie befürchten, den neuen Anforderungen möglicherweise nicht gerecht werden zu können. Sie sind in Sorge, dass sie ihren Arbeitsplatz möglicherweise verlieren werden und/ oder Einkommens-Einbußen hinnehmen müssen. Sie werden deshalb versuchen, die von der Geschäftsführung geplanten Entscheidungen zu verhindern und/ oder deren Verwirklichung zu unterlaufen.

Ein Teil dieser Gruppe wird ihre Haltung im Einklang mit der eher skeptischen Grundhaltung der zweiten Gruppe von Krankenhaus-, Kunden" und sich damit in ihrer eignen Sichtweise bestärkt sehen. Sie werden deshalb auch unter Einbeziehung der Reaktion der „Kunden“ bei ihrer ablehnenden Haltung bleiben. Eine zweite Teil-Gruppe von Mitarbeitern sieht sich mit ihrer Haltung im Widerspruch zu den Erwartungen der 
ersten Kunden-Gruppe und hat deshalb das Problem, sich entscheiden zu müssen, welches ihrer beiden Individualziele ihre Haltung zu den Plänen der Geschäftsführung entscheidend prägen soll. So ist es durchaus denkbar, dass sie ihre Bedenken bezüglich der Befriedigung ihrer persönlichen Bedürfnisse zurückstellen und sich zu Gunsten ihrer Patienten für die Unterstützung der Pläne der Geschäftsführung entscheiden.

- Eine andere Gruppe von Mitarbeitern versteht die geplanten Änderungen nicht als Bedrohung, sondern als Chance, vor allem aber als Chance für die langfristige finanzielle Sicherung des Krankenhauses und der von inm vorgehaltenen Arbeitsplätzen. Sie erhoffen sich im Gegensatz zu der ersten Mitarbeiter-Gruppe ein erhöhtes $\mathrm{Ma} B$ an Befriedigung ihres Bedürfnisses nach Sicherheit; dabei gehen sie davon aus, dass mittels der geplanten Maßnahmen zwar nicht jeder Arbeitsplatz, in jedem Falle aber ihr eigener langfristig gesichert ist. Sie erwarten auf der Grundlage neuer Strukturen und Arbeitsabläufe die Zunahme der Befriedigung ihres Bedürfnisses nach Selbstverwirklichung und Selbstentfaltung und werden deshalb die Pläne der Geschäftsführung unterstützen.

Aber auch die Mitglieder dieser Mitarbeitergruppe sind sich wegen ihrer Agentenrolle in ihrer Haltung zu den Plänen der Geschäftsführung nicht einig. Je nachdem, welches ihrer beiden Individualziele ihre Reaktion prägt, wird es auch in dieser Gruppe Vertreter geben, die bei der schon skizzierten Haltung bleiben, während sich andere doch noch im Sinne der eher skeptischen Grundhaltung der zweiten „Kunden“-Gruppe umorientieren und die Pläne der Geschäftsführung ablehnen.

\section{Führungskräfte}

Auch die Führungskräfte sind uneins in ihrer Haltung zu den Plänen der Geschäftsführung. Einerseits werden sie in Loyalität zu ihren Mitarbeitern, aber auch, weil sie deren Argumente akzeptieren, wie diese reagieren - also einerseits zustimmend und zum anderen ablehnend.

Auch die Führungskräfte sind Agenten der Patienten und werden deshalb ihre Haltung anhand der unterschiedlichen Reaktionen der beiden „Kunden“-Gruppen überprüfen. Sie werden sich - wie ihre Mitarbeiter - entweder in ihrer ablehnenden Haltung bestärkt sehen oder unter Hintanstellen ihrer Befürchtungen bezüglich der Konsequenzen für sie persönlich der Geschäftsführung helfen, deren Pläne zu realisieren.

\section{Krankenkassen}

Die Krankenkassen haben ein großes Interesse daran, dass die Qualität der stationären Behandlung trotz der Verknappung der zur Verfügung stehenden Mittel mindestens aufrechterhalten wird. Sie werden deshalb die Geschäftsführung bei der Realisierung der geplanten Veränderungen unterstützen.

\section{Eigentümer}

Der Eigentümer des Krankenhauses wird in Abhängigkeit davon, ob er sein Engagement als ein kurz- und mittelfristiges sieht oder ob er sich auf längere Zeit binden will, reagieren. Eine eher kurz-/mittelfristige Orientierung führt zu einer Ablehnung der Pläne der Geschäftsführung und zu dem Versuch, die Geschäftsführung zu den Alternativ-Strategien zu bewegen. Der Eigentümer, der sich längerfristig engagiert, wird die Geschäftsführung bei der Realisierung der Alternative 4. unterstützen. 